\title{
Unexpected reaction products of uracil and its methyl derivatives with acetic anhydride and methylene chloride
}

Olga Michalak, ${ }^{*[a]}$ Piotr Cmoch ${ }^{[\mathrm{b}]}$ Andrzej Leś, ${ }^{[\mathrm{c}]}$ Marcin Cybulski, ${ }^{[\mathrm{a}]}$ Piotr Krzeczyński, ${ }^{[\mathrm{a}]}$ Kinga Trzcińska ${ }^{[\mathrm{d}]}$ Przemysław Miszta ${ }^{[\mathrm{e}]}$ Pakhuri Mehta ${ }^{[\mathrm{e}]}$ Sławomir Filipek ${ }^{[\mathrm{e}]}$

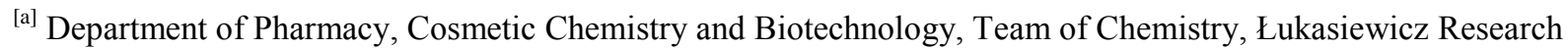
Network-Industrial Chemistry Institute, 8 Rydygiera Str., 01-793 Warsaw, Poland

${ }^{[b]}$ Institute of Organic Chemistry Polish Academy of Sciences, 44/52 Kasprzaka street, 01-224 Warsaw, Poland

${ }^{[c]}$ Faculty of Chemistry, University of Warsaw, 02-093 Warsaw, Poland

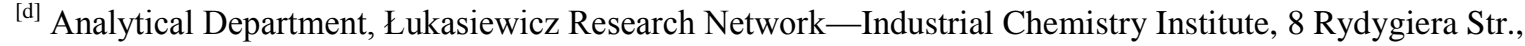
01-793 Warsaw, Poland

${ }^{[\mathrm{e}]}$ Faculty of Chemistry, Biological and Chemical Research Centre, University of Warsaw, 02-093 Warsaw, Poland

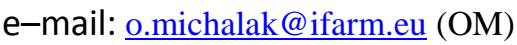




\section{Table of Content:}

Table S1. The ${ }^{1} \mathrm{H},{ }^{13} \mathrm{C}$ and ${ }^{15} \mathrm{~N}$ NMR chemical shifts of products obtained from thymine (procedure A and B) . . . . . . . . . . . . . . . . . . . . . . . . . . . . . . . . S4

Table S2. The ${ }^{1} \mathrm{H},{ }^{13} \mathrm{C}$ and ${ }^{15} \mathrm{~N}$ NMR chemical shifts of $\mathrm{U}$ and 6-MeU acetylation products

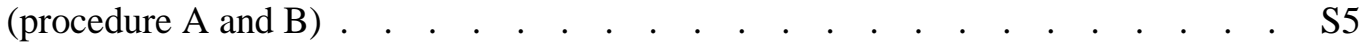

Table S3. The ${ }^{1} \mathrm{H},{ }^{13} \mathrm{C}$ and ${ }^{15} \mathrm{~N}$ NMR chemical shifts of 6-methyluracyl acetylation products with $\mathrm{CH}_{2} \mathrm{Cl}_{2}$ as solvent and reagent (procedure $\mathrm{B} \mathrm{I} \mathrm{C)} \mathrm{.} \mathrm{.} \mathrm{.} \mathrm{.} \mathrm{.} \mathrm{.} \mathrm{.} \mathrm{.} \mathrm{.} \mathrm{.} \mathrm{.} \mathrm{.} \mathrm{S6}$

Table S4. The ${ }^{1} \mathrm{H},{ }^{13} \mathrm{C}$ and ${ }^{15} \mathrm{~N}$ NMR chemical shifts of thymine acetylation products with $\mathrm{Cl}-\mathrm{CH}_{2}-\mathrm{CH}_{2}-\mathrm{Cl}$ as solvent and reagent (procedure E) . . . . . . . . . . . $\mathrm{S} 7$

Table S5. The ${ }^{1} \mathrm{H},{ }^{13} \mathrm{C}$ and ${ }^{15} \mathrm{~N}$ NMR chemical shifts of uracil acetylation products with $\mathrm{Cl}-\mathrm{CH}_{2}-\mathrm{CH}_{2}-\mathrm{Cl}$ as solvent and reagent (procedure E) . . . . . . . . . . $\mathrm{S} 8$

Table S6. The ${ }^{1} \mathrm{H},{ }^{13} \mathrm{C}$ and ${ }^{15} \mathrm{~N}$ NMR chemical shifts of thymine acetylation products (procedure $\mathrm{F}, \mathrm{G}$ and $\mathrm{H}$ ) . . . . . . . . . . . . . . . . . . . . . . . . . . . . . . . . S9

Table S7. The ${ }^{1} \mathrm{H},{ }^{13} \mathrm{C}$ and ${ }^{15} \mathrm{~N}$ NMR chemical shifts of thymine acetylation products (procedure I) . . . . . . . . . . . . . . . . . . . . . . . . . . . . . S10

Table S8. The ${ }^{1} \mathrm{H},{ }^{13} \mathrm{C}$ and ${ }^{15} \mathrm{~N}$ NMR chemical shifts of uracyl acetylation products with $\mathrm{CD}_{2} \mathrm{Cl}_{2}$ as solvent and reagent (procedure I) . . . . . . . . . . . . $\mathrm{S} 11$

Table S9. Crystal data and structure refinement for investigated compound . . . . . . S12

Table S10. The geometry of the hydrogen bonds for the 296-55-3-2 . . . . . . . . S13

Figure S1. Supramolecular architecture of investigated compound in the crystal, viewed along $a$-direction. The $\mathrm{H}$ atoms were omitted for clarity . . . . . . S13

Figure S2. Supramolecular architecture of investigated compound in the crystal, viewed along $b$-direction. The $\mathrm{H}$ atoms were omitted for clarity . . . . . . . S14

Figure S3. Supramolecular architecture of investigated compound in the crystal, viewed along $c$-direction. The $\mathrm{H}$ atoms were omitted for clarity . . . . . . S14

Table S11. Bond lengths for investigated compound . . . . . . . . . . . . . . S15

Table S12. Valence angles for investigated compound . . . . . . . . . . . . . S15

Table S13. Torsion angles for investigated compound . . . . . . . . . . . . . S15

Table S14. Coordinates Atom Positions . . . . . . . . . . . . . . . . . . $\quad$ S16

Table S15. Yield of selected reactions with $\mathrm{Ac}_{2} \mathrm{O}$.

Table S16. Yield of selected reactions with $\left(\mathrm{C}_{2} \mathrm{H}_{5} \mathrm{OC}\right)_{2} \mathrm{O}$ and with $\mathrm{Ac}_{2} \mathrm{O}$ in $\mathrm{CD}_{2} \mathrm{Cl}_{2} \quad$. . . $\quad \mathrm{S} 18$

Figure S4. The acetyloxydiethylamine ( $N^{1}$-acetyloxyethyl diethylamine) appearing in the Step3, Scheme 4 of the proposed mechanism of acetylation (see the text) 
Figure S5. The transition state species with four-member ring $\mathrm{N}-\mathrm{C}-\mathrm{O}-\mathrm{C}$ appearing in the Step3, Scheme 4 of the proposed mechanism of acetylation (see the text)

Figure S6. The acetaldehyde (III) appearing in the Step3, Scheme 4 of the proposed mechanism of acetylation (see the text) . . . . . . . . . . . . . . S20

Figure S7. The acetyldiethylamine (IV) appearing in the Step3, Scheme 4 of the proposed mechanism of acetylation (see the text) . . . . . . . . . . . . . . S20

Table S17 Reaction, uphill and downhill transition states as well as Eq. 2 details (Filenames correspond to the Cartesian coordinate files in the Table S18). . . S21

Table S18 The Cartesian coordinates $[\AA]$. . . . . . . . . . . . . . . . . . . . . . . . . S22

Details of reactions considered in the text. . . . . . . . . . . . . . . . . . S28

References . . . . . . . . . . . . . . . . . . . . . . . . . . . . $\mathrm{S} 30$ 
Table S1. The ${ }^{1} \mathrm{H},{ }^{13} \mathrm{C}$ and ${ }^{15} \mathrm{~N}$ NMR (all in $\mathrm{CDCl}_{3}, 25^{\circ} \mathrm{C}$ ) chemical shifts of products obtained from thymine (procedure A and $\mathrm{B}$ ).

\begin{tabular}{|c|c|c|c|c|c|c|}
\hline & $2 a$ & $2 \mathbf{b}^{\mathrm{b}}$ & $2 c^{b}$ & $2 e$ & $2 f^{c}$ & $2 g^{c}$ \\
\hline $\mathrm{H} 1 / \mathrm{N} 1$ & $---/-207.7$ & $---/-243.0$ & $---/-236.2$ & $9.63 /-256.1$ & $---/^{d}$ & $---/^{d}$ \\
\hline C2 & 149.4 & 150.7 & 149.6 & 152.4 & 150.9 & 149.7 \\
\hline $\mathrm{H} 3 / \mathrm{N} 3$ & $8.63 /-226.2$ & $9.06 /-226.4$ & $8.94 /-226.8$ & $---/^{a}$ & $--/^{d}$ & $--/^{d}$ \\
\hline C4 & 163.3 & 163.8 & 163.5 & 163.1 & 162.5 & 162.2 \\
\hline $\mathrm{H} 5 / \mathrm{C} 5$ & $---/ 113.2$ & $---/ 111.4$ & $---/ 111.8$ & $\begin{array}{l}--/ 110.4 \\
\end{array}$ & $---/ 110.7$ & $\begin{array}{l}--/ 111.1 \\
\end{array}$ \\
\hline $\mathrm{H} 6 / \mathrm{C} 6$ & $8.06 / 133.0$ & $7.29 / 140.0$ & $7.13 / 134.7$ & $7.09 / 135.2$ & $7.31 / 139.1$ & $7.14 / 133.8$ \\
\hline $\mathrm{CH}_{3}$ & $1.99 / 12.5$ & $1.90 / 12.2$ & $1.94 / 12.6$ & $1.95 / 12.8$ & $1.91 / 12.8$ & $1.94 / 13.1$ \\
\hline $\mathrm{CH}_{3} \mathrm{CO}$ & $2.74 / 27.2 \mid 169.5$ & ----- & ----- & ---- & $\begin{array}{l}---- \\
-1\end{array}$ & ----- \\
\hline$N^{1}-\mathrm{CH}_{2} \mathrm{OCOCH}_{3}$ & ----- & $\begin{array}{c}5.64 / 69.4|171.0| \\
2.11 / 20.7 \\
\end{array}$ & ---- & ---- & $\begin{array}{c}5.64 / 70.2|170.9| \\
2.10 / 20.7^{d} \\
\end{array}$ & ---- \\
\hline $\begin{array}{c}N^{1}-\mathrm{CHCOCH}_{3} \\
\stackrel{1}{\mathrm{CH}_{3}}\end{array}$ & ---- & ---- & $\begin{array}{c}6.95 / 75.2 \mid 1.59 / 19.5 \\
168.6 \mid 2.08 / 20.7\end{array}$ & ---- & ---- & $\begin{array}{c}6.96 / 75.8|1.58 / 19.5| \\
168.5 \mid 2.07 / 20.7^{\mathrm{e}}\end{array}$ \\
\hline$N^{3}-\mathrm{CH}_{2} \mathrm{OCOCH}_{3}$ & ----- & ----- & ----- & $\begin{array}{c}5.98 / 64.1 \mid \\
169.9 \mid 2.09 / 20.8\end{array}$ & $\begin{array}{c}5.94 / 64.7|169.7| \\
2.03 / 20.6^{\mathrm{e}} \\
\end{array}$ & $\begin{array}{c}5.90 / 64.8|169.8| \\
2.03 / 20.7^{\mathrm{e}} \\
\end{array}$ \\
\hline
\end{tabular}

${ }^{a}$ no ${ }^{15} \mathrm{~N}$ signal in the ${ }^{1} \mathrm{H}-{ }^{15} \mathrm{~N}$ g-HMBC experiment, ${ }^{\mathrm{b}}{ }^{1} \mathrm{H} /{ }^{13} \mathrm{C} /{ }^{15} \mathrm{~N}$ NMR chemical shifts for mixture of $\mathbf{2 b}$ and $2 \mathbf{c}$, ${ }^{\mathrm{c}}{ }^{1} \mathrm{H} /{ }^{13} \mathrm{C} /{ }^{15} \mathrm{~N} \mathrm{NMR}$ chemical shifts for mixture of $\mathbf{2 f}$ and $\mathbf{2 g},{ }^{\mathrm{d} 15} \mathrm{~N}$ NMR spectra not measured, ${ }^{\mathrm{e}}$ signals of $\mathrm{CH}_{3}$ singlet of acetyl groups overlap 
Table S2. The ${ }^{1} \mathrm{H},{ }^{13} \mathrm{C}$ and ${ }^{15} \mathrm{~N}$ NMR (all in $\mathrm{CDCl}_{3}, 25^{\circ} \mathrm{C}$ ) chemical shifts of $\mathrm{U}$ and 6-MeU acetylation products (procedure A and $\mathrm{B}$ ).

\begin{tabular}{|c|c|c|c|c|c|c|}
\hline & $3 a$ & $3 b^{a}$ & $3 c$ & $3 e^{a}$ & $3 f^{b}$ & $3 g^{b}$ \\
\hline $\mathrm{H} 1 / \mathrm{N} 1$ & $---/-206.7$ & $---/-240.4$ & $---/-233.6$ & $9.41 /-254.7$ & $---/-241.8$ & $---/^{c}$ \\
\hline $\mathrm{C} 2$ & 149.1 & 150.3 & 149.5 & 152.0 & 150.8 & 149.7 \\
\hline $\mathrm{H} 3 / \mathrm{N} 3$ & $8.51 /-224.7$ & $8.77 /-224.8$ & $9.21 /-224.6$ & $---/-212.6$ & $---/-214.0$ & $---/-214.2$ \\
\hline C4 & 162.2 & 162.9 & 162.9 & 162.1 & 161.4 & 161.3 \\
\hline $\mathrm{H} 5 / \mathrm{C} 5$ & $5.90 / 104.8$ & $5.74 / 102.9$ & $5.80 / 103.4$ & $5.81 / 102.1$ & $5.79 / 102.3$ & $5.84 / 102.8$ \\
\hline $\mathrm{H} 6 / \mathrm{C} 6$ & $8.23 / 137.4$ & $7.51 / 144.4$ & $7.35 / 139.1$ & $7.22 / 139.2$ & $7.51 / 143.3$ & $7.34 / 138.0$ \\
\hline $\mathrm{CH}_{3} \mathrm{CO}$ & $\begin{array}{c}2.76 / 27.1 \\
169.3\end{array}$ & ---- & ---- & ---- & ---- & ---- \\
\hline $\begin{array}{l}N^{1}-\mathrm{CH}_{2} \mathrm{O} \\
\mathrm{COCH}_{3}\end{array}$ & ---- & $\begin{array}{c}5.66 / 69.5 \\
171.0 \mid 2.14 / 20.7 \\
\end{array}$ & ---- & ---- & $\begin{array}{c}5.68 / 70.3 \\
171.0 \mid 2.08 / 20.7^{d} \\
\end{array}$ & ----- \\
\hline $\begin{array}{c}N^{1}-\mathrm{CHCOCH}_{3} \\
\mathrm{CH}_{3}\end{array}$ & ---- & ---- & $\begin{array}{c}6.95 / 75.4|168.5| 2.11 / 20.7 \\
1.62 / 19.5\end{array}$ & ---- & ---- & $\begin{array}{c}6.97 / 76.0|1.62 / 19.5| 168.5 \mid \\
2.08 / 20.7^{d}\end{array}$ \\
\hline $\begin{array}{l}N^{3}-\mathrm{CH}_{2} \mathrm{O} \\
\mathrm{COCH}_{3}\end{array}$ & ---- & --.-- & --.-- & $\begin{array}{c}5.96 / 63.8 \\
169.9 \mid 2.09 / 20.7 \\
\end{array}$ & $\begin{array}{c}5.96 / 64.4 \\
169.7 \mid 2.14 / 20.7^{d} \\
\end{array}$ & $\begin{array}{c}5.95 / 64.5 \\
169.8 \mid 2.11 / 20.7^{d} \\
\end{array}$ \\
\hline
\end{tabular}

${ }^{\text {a } 1} \mathrm{H} /{ }^{13} \mathrm{C} /{ }^{15} \mathrm{~N}$ NMR chemical shifts for mixture $\mathbf{3 b}$ and $\mathbf{3 e},{ }^{\mathrm{b}}{ }^{1} \mathrm{H} /{ }^{13} \mathrm{C} /{ }^{15} \mathrm{~N}$ NMR chemical shifts for mixture $\mathbf{3 f}$ and $\mathbf{3 g}$, ${ }^{\mathrm{c}}{ }^{15} \mathrm{~N}$ signal not observed in the ${ }^{1} \mathrm{H}-{ }^{15} \mathrm{~N}$ HMBC experiment, ${ }^{\mathrm{d}}$ signals of $\mathrm{CH}_{3}$ singlet of acetyl groups overlap 
Table S3. The ${ }^{1} \mathrm{H},{ }^{13} \mathrm{C}$ and ${ }^{15} \mathrm{~N}$ NMR (all in $\mathrm{CDCl}_{3}, 25^{\circ} \mathrm{C}$ ) chemical shifts of 6-methyluracyl acetylation products with $\mathrm{CH}_{2} \mathrm{Cl}_{2}$ as solvent and reagent (procedure B I C)

\begin{tabular}{|c|c|c|c|}
\hline & If & $1 b^{a}$ & $1 e^{a}$ \\
\hline H1/N1 & $---/-240.5$ & $---/-239.5$ & $9.0 /-247.8$ \\
\hline $\mathrm{C} 2$ & 151.3 & 150.8 & 151.7 \\
\hline $\mathrm{H} 3 / \mathrm{N} 3$ & $---/-216.7$ & $8.44 /^{b}$ & $--/^{b}$ \\
\hline C4 & 160.6 & 161.7 & 162.0 \\
\hline $\mathrm{H} 5 / \mathrm{C} 5$ & $5.69 / 102.7$ & $5.62 / 103.3$ & $5.60 / 100.3$ \\
\hline $\mathrm{H} 6 / \mathrm{C} 6$ & $---/ 151.5$ & 152.6 & 150.4 \\
\hline $\mathrm{CH}_{3}$ & $2.31 / 19.3$ & $2.31 / 19.3$ & $2.17 / 19.1$ \\
\hline $\mathrm{CH}_{3} \mathrm{CO}$ & ----- & ----- & ----- \\
\hline $\begin{array}{l}N^{1}-\mathrm{CH}_{2} \mathrm{O} \\
\mathrm{COCH}_{3}\end{array}$ & $\begin{array}{c}5.87 / 67.4 \\
169.6 \mid 2.13 / 20.7 \text { * }\end{array}$ & $\begin{array}{c}5.85 / 66.7 \\
169.7 \mid 2.12 / 20.7 *\end{array}$ & ----- \\
\hline $\begin{array}{c}N^{1}-\mathrm{CHCOCH}_{3} \\
\mathrm{CH}_{3}\end{array}$ & ----- & ----- & ----- \\
\hline $\begin{array}{l}N^{3}-\mathrm{CH}_{2} \mathrm{O} \\
\mathrm{COCH}_{3}\end{array}$ & $\begin{array}{c}5.95 / 64.6 \\
169.7 \mid 2.07 / 20.7 *\end{array}$ & ----- & $\begin{array}{c}5.94 / 63.8 \\
169.8 / 2.07 / 20.7 *\end{array}$ \\
\hline
\end{tabular}

$*{ }^{13} \mathrm{C}$ signals of both methyl groups overlap, ${ }^{a}{ }^{1} \mathrm{H} /{ }^{13} \mathrm{C} /{ }^{15} \mathrm{~N}$ NMR chemical shifts for mixture of $\mathbf{1 b}$ and $\mathbf{1 e}$,

${ }^{b}{ }^{15} \mathrm{~N}$ NMR chemical shifts not observed in the ${ }^{1} \mathrm{H}^{-15} \mathrm{~N}$ HMBC experiment. 
Table S4. The ${ }^{1} \mathrm{H},{ }^{13} \mathrm{C}$ and ${ }^{15} \mathrm{~N}$ NMR (all in $\mathrm{CDCl}_{3}, 25^{\circ} \mathrm{C}$ ) chemical shifts of thymine acetylation products with $\mathrm{Cl}-\mathrm{CH}_{2}-\mathrm{CH}_{2}-\mathrm{Cl}$ as solvent and reagent (procedure $\mathrm{E}$ )

\begin{tabular}{|c|c|c|c|c|}
\hline & $2 c$ & $2 i$ & $2 j$ & 21 \\
\hline $\mathrm{H} 1 / \mathrm{N} 1$ & $---/-236.2$ & $9.87 /-256.7$ & $9.80 /-255.7$ & $---/-237.5$ \\
\hline C2 & 149.6 & 153.1 & 153.1 & 150.1 \\
\hline $\mathrm{H} 3 / \mathrm{N} 3$ & $8.78 /-226.4$ & $---/-219.2$ & $---/-219.7$ & $---/-220.5$ \\
\hline C4 & 163.3 & 163.7 & 163.9 & 162.8 \\
\hline $\mathrm{H} 5 / \mathrm{C} 5$ & $---/ 111.8$ & $---/ 110.3$ & $---/ 110.2$ & $---/ 111.0$ \\
\hline $\mathrm{H} 6 / \mathrm{C} 6$ & $7.15 / 134.7$ & $7.06 / 134.7$ & $7.05 / 134.5$ & $7.14 / 133.0$ \\
\hline $\mathrm{CH}_{3}$ & $1.96 / 12.6$ & $1.94 / 12.9$ & $1.93 / 13.0$ & $1.96 / 13.3$ \\
\hline $\begin{array}{c}N^{1}-\mathrm{CHOCOCH}_{3} \\
\quad \mathrm{CH}_{3} \\
\mathrm{CH}_{3}\end{array}$ & $\begin{array}{c}6.96 / 75.2|168.6| 2.10 / 20.7 \\
1.60 / 19.57\end{array}$ & ---- & ---- & $\begin{array}{c}7.00 / 75.8|168.6 / 2.09 / 20.8| \\
1.60 / 19.5\end{array}$ \\
\hline$N^{3}-\mathrm{CH}_{2}-\mathrm{CH}_{2}-\mathrm{Cl}$ & ---- & $\begin{array}{l}4.31 / 41.7 \\
3.75 / 40.0 \\
\end{array}$ & ---- & $\begin{array}{l}4.30 / 42.3 \\
3.70 / 39.9 \\
\end{array}$ \\
\hline$N^{3}-\mathrm{CH}_{2}-\mathrm{CH}_{2}-\mathrm{OCOCH}_{3}$ & ---- & ----- & $\begin{array}{c}4.23 / 39.5|4.32 / 61.1| \\
171.0 \mid 2.02 / 20.9 \\
\end{array}$ & ----- \\
\hline
\end{tabular}


Table S5. The ${ }^{1} \mathrm{H},{ }^{13} \mathrm{C}$ and ${ }^{15} \mathrm{~N}$ NMR (all in $\mathrm{CDCl}_{3}, 25^{\circ} \mathrm{C}$ ) chemical shifts of uracil acetylation products with $\mathrm{Cl}-\mathrm{CH}_{2}-\mathrm{CH}_{2}-\mathrm{Cl}$ as solvent and reagent (procedure $\mathrm{E}$ )

\begin{tabular}{|c|c|c|c|c|c|c|}
\hline & 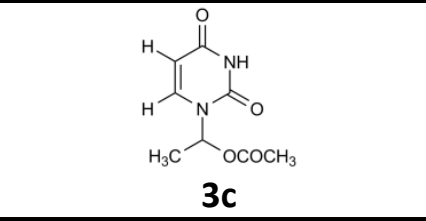 & $3 i^{\text {a }}$ & $\mathbf{3 j}$ & 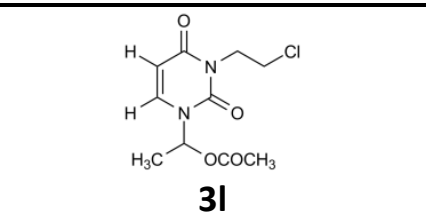 & 等 & $\underbrace{3 n}$ \\
\hline $\mathrm{H} 1 / \mathrm{N} 1$ & $---/-233.4$ & $10.2 /-252.5$ & $9.69 /-252.8$ & $---/-234.5$ & $---/-234.6$ & $---/-250.2$ \\
\hline $\mathrm{C} 2$ & 149.5 & 152.8 & 152.9 & 150.1 & 150.3 & 151.4 \\
\hline $\mathrm{H} 3 / \mathrm{N} 3$ & $9.21 /-224.7$ & $---/-217.2$ & $---/-217.5$ & $---/-218.6$ & $---/-219.4$ & $---/-218.5$ \\
\hline C4 & 162.9 & 163.2 & 163.1 & 162.0 & 162.2 & 162.8 \\
\hline $\mathrm{H} 5 / \mathrm{C5}$ & $5.80 / 103.4$ & $5.73 / 101.6$ & $5.79 / 102.1$ & $5.83 / 102.8$ & $5.81 / 102.8$ & $5.75 / 101.3$ \\
\hline $\mathrm{H} 6 / \mathrm{C} 6$ & $7.35 / 139.1$ & $7.25 / 139.4$ & $7.19 / 138.5$ & $7.32 / 137.3$ & $7.30 / 137.1$ & $7.21 / 143.3$ \\
\hline $\begin{array}{c}N^{1}-\mathrm{CHCOCH}_{3} \\
\mathrm{CH}_{3}\end{array}$ & $\begin{array}{c}6.96 / 75.4|168.6| 2.11 / 20.7 \mid \\
1.62 / 19.5\end{array}$ & ----- & ----- & $\begin{array}{c}6.99 / 76.0|168.5| 2.11 / 20.7 \mid \\
1.62 / 19.5\end{array}$ & $\begin{array}{c}6.99 / 76.0|168.5| 2.10 / 20.7^{*} \\
\mid 1.61 / 19.5\end{array}$ & ----- \\
\hline$N^{3}-\mathrm{CH}_{2} \mathrm{OCOCH}_{3}$ & ---- & ---- & ---- & $\begin{array}{l}---- \\
\end{array}$ & $\begin{array}{l}---- \\
\end{array}$ & ----- \\
\hline$N^{1}-\mathrm{CH}_{2}-\mathrm{CH}_{2}-\mathrm{Cl}$ & $\begin{array}{l}---- \\
--\end{array}$ & $\begin{array}{l}---- \\
--\end{array}$ & ---- & ---- & $\begin{array}{l}---- \\
--\end{array}$ & $4.05 / 51.9 \mid 3.82 / 41.9$ \\
\hline$N^{3}-\mathrm{CH}_{2}-\mathrm{CH}_{2}-\mathrm{Cl}$ & ----- & $4.28 / 41.4 \mid 3.71 / 40.0$ & ----- & $4.30 / 42.1 \mid 3.73 / 39.8$ & ----- & \\
\hline$N^{3}-\mathrm{CH}_{2}-\mathrm{CH}_{2}-\mathrm{OCOCH}_{3}$ & ----- & ---- & $\begin{array}{c}4.22 / 39.4 \mid 4.33 / 61.1 \\
171.0 \mid 2.03 / 20.8\end{array}$ & ----- & $\begin{array}{c}4.19+4.27 / 40.1|4.31| 61.1 \\
171.0 \mid 2.03 / 20.8 *\end{array}$ & $\begin{array}{c}4.24 / 40.0|4.32 / 61.1| \\
171.0 \mid 2.02 / 20.8\end{array}$ \\
\hline
\end{tabular}

${ }^{\mathrm{a}}{ }^{1} \mathrm{H} /{ }^{13} \mathrm{C} /{ }^{15} \mathrm{~N}$ NMR chemical shifts for mixture of $\mathbf{3 c}$ and $\mathbf{3 i},{ }^{\text {b }} \mathrm{H} /{ }^{13} \mathrm{C} /{ }^{15} \mathrm{~N}$ NMR chemical shifts for mixture of $\mathbf{3 m}$ and $\mathbf{3 g}$,

${ }^{*} \mathrm{C}$ signals of both methyl groups overlap 
Table S6. The ${ }^{1} \mathrm{H},{ }^{13} \mathrm{C}$ and ${ }^{15} \mathrm{~N}$ NMR (all in $\mathrm{CDCl}_{3}, 25^{\circ} \mathrm{C}$ ) chemical shifts of thymine acetylation products (procedure $\mathrm{F}, \mathrm{G}$ and $\mathrm{H}$ ).

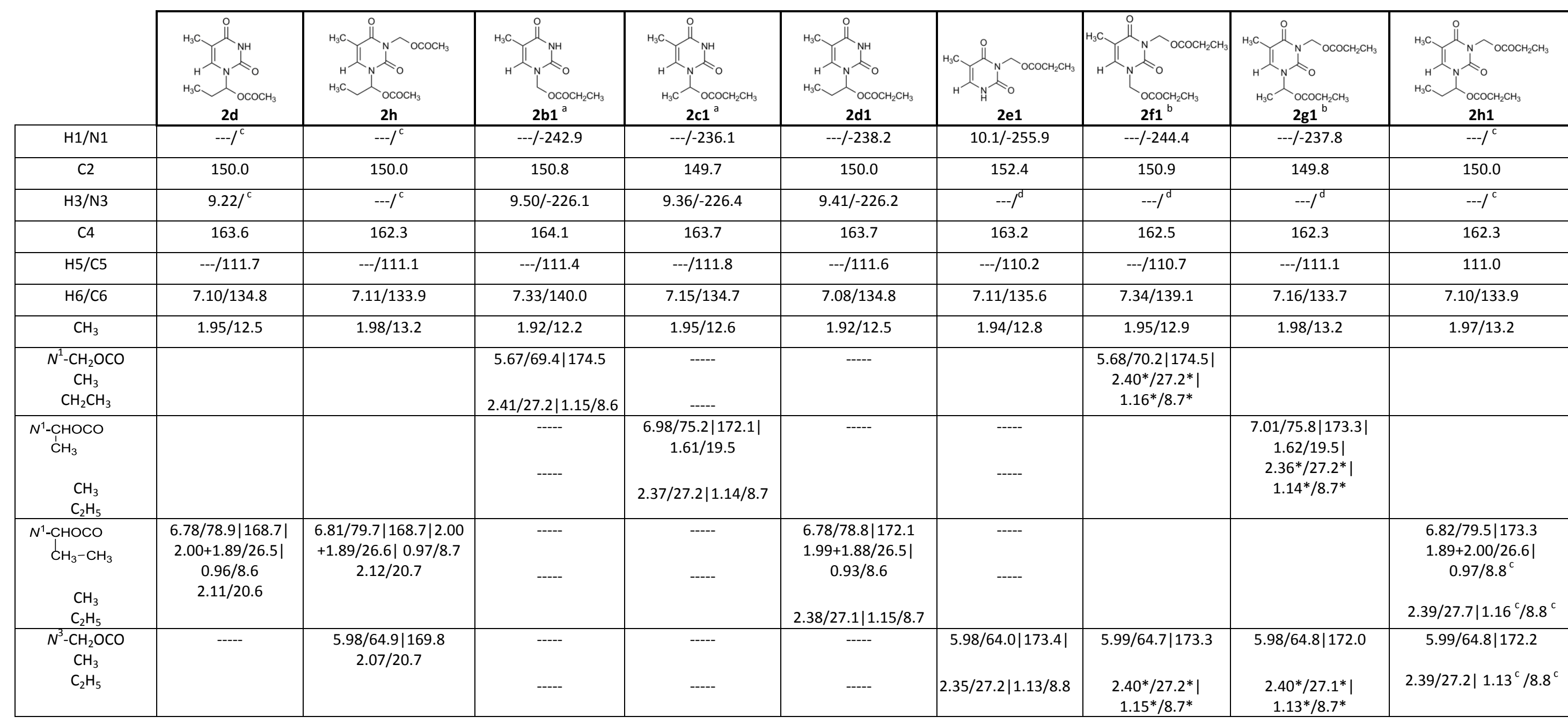

${ }^{\mathrm{a}}{ }^{1} \mathrm{H} /{ }^{13} \mathrm{C} /{ }^{15} \mathrm{~N}$ NMR chemical shifts given in a mixture $\mathbf{2 b 1}$ and $\mathbf{2 c 1},{ }^{\mathrm{b}}{ }^{1} \mathrm{H} /{ }^{13} \mathrm{C} /{ }^{15} \mathrm{~N}$ NMR chemical shifts given in a mixture $\mathbf{2 f 1}$ and $\mathbf{2 g 1}$,

${ }^{c}{ }^{15} \mathrm{~N}$ chemical shifts not measured, ${ }^{\mathrm{d}}{ }^{15} \mathrm{~N}$ NMR chemical shifts not observed in the ${ }^{1} \mathrm{H}-{ }^{15} \mathrm{~N}$ HMBC experiments,

$*{ }^{1} \mathrm{H} /{ }^{13} \mathrm{C}$ signals of both ethyl groups partially overlap and that is why can be assigned reversibly. 
Table S7. The ${ }^{1} \mathrm{H},{ }^{13} \mathrm{C}$ and ${ }^{15} \mathrm{~N}$ NMR (all in $\mathrm{CDCl}_{3}, 25^{\circ} \mathrm{C}$ ) chemical shifts of thymine acetylation products (procedure I)

\begin{tabular}{|c|c|c|c|}
\hline & 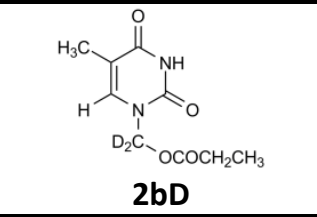 & $2 \mathrm{eD}$ & 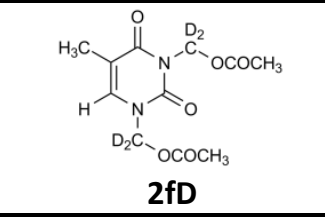 \\
\hline $\mathrm{H} 1 / \mathrm{N} 1$ & $--/^{a}$ & $8.96 /^{\mathrm{a}}$ & $--/^{a}$ \\
\hline $\mathrm{C} 2$ & 150.4 & 151.9 & 150.9 \\
\hline $\mathrm{H} 3 / \mathrm{N} 3$ & $8.16 /^{\mathrm{a}}$ & $--/^{\mathrm{a}}$ & $--/^{a}$ \\
\hline C4 & 163.3 & 163.1 & 162.5 \\
\hline $\mathrm{H} 5 / \mathrm{C5}$ & $---/ 111.4$ & $---/ 110.4$ & $---/ 110.8$ \\
\hline $\mathrm{H} 6 / \mathrm{C} 6$ & $7.30 / 139.9$ & $7.06 / 134.9$ & $7.34 / 139.0$ \\
\hline $\mathrm{CH}_{3}$ & $1.93 / 12.3$ & $1.96 / 12.8$ & $1.95 / 12.9$ \\
\hline$N^{1}-\mathrm{CD}_{2} \mathrm{OCOCH}_{3}$ & ${ }^{\mathrm{b}} / \mathrm{b}|171.0| 2.14 / 20.7$ & ----- & ${ }^{b} /{ }^{b}|171.0| 2.14 / 20.7^{*}$ \\
\hline$N^{3}-\mathrm{CD}_{2} \mathrm{OCOCH}_{3}$ & ----- & ${ }^{\mathrm{b}} / \mathrm{b}|169.9| 2.10 / 20.7$ & ${ }^{\mathrm{b}} /^{b}|169.8| 2.08 / 20.7^{*}$ \\
\hline
\end{tabular}

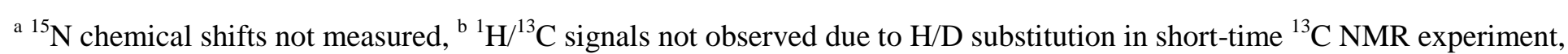

$*{ }^{1} \mathrm{H} /{ }^{13} \mathrm{C}$ signals of both methyl groups overlap 
Table S8. The ${ }^{1} \mathrm{H},{ }^{13} \mathrm{C}$ and ${ }^{15} \mathrm{~N} \mathrm{NMR}$ (all in $\mathrm{CDCl}_{3}, 25^{\circ} \mathrm{C}$ ) chemical shifts of uracyl acetylation products with $\mathrm{CD}_{2} \mathrm{Cl}_{2}$ as solvent and reagent (procedure I)

\begin{tabular}{|c|c|c|c|c|c|}
\hline & 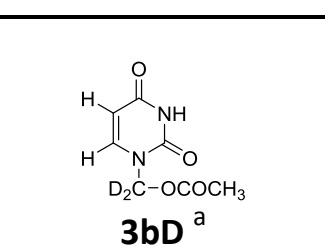 & $\begin{array}{c}\mathrm{H}_{3} \mathrm{C}-\mathrm{OCOCH}_{3} \\
3 \mathrm{c}^{\mathrm{a}}\end{array}$ & 3ed & 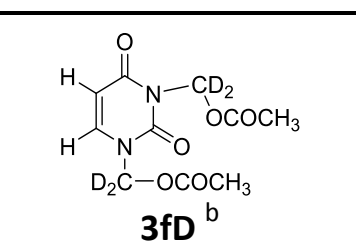 & $\begin{array}{c}\mathrm{SgD}^{\mathrm{b}} \\
\mathrm{O}\end{array}$ \\
\hline $\mathrm{H} 1 / \mathrm{N} 1$ & $--/^{d}$ & $--/^{d}$ & $8.32 /^{d}$ & $--/^{d}$ & $-/^{\mathrm{d}}$ \\
\hline $\mathrm{C} 2$ & 150.3 & 149.3 & 152.1 & 150.8 & 149.7 \\
\hline $\mathrm{H} 3 / \mathrm{N} 3$ & $8.68^{c} /^{d}$ & $8.58^{c} /^{d}$ & $--/^{d}$ & $--/^{\mathrm{d}}$ & $--/^{\mathrm{d}}$ \\
\hline C4 & 162.8 & 162.5 & 162.0 & 161.4 & 161.2 \\
\hline $\mathrm{H} 5 / \mathrm{C} 5$ & $5.73 / 102.9$ & $5.79 / 103.4$ & $5.80 / 102.1$ & $5.79 / 102.4$ & $5.85 / 102.8$ \\
\hline $\mathrm{H} 6 / \mathrm{C} 6$ & $7.50 / 144.3$ & $7.33 / 139.1$ & $7.19 / 138.8$ & $7.51 / 143.3$ & $7.34 / 138.0$ \\
\hline$N^{1}-\mathrm{CD}_{2} \mathrm{OCOCH}_{3}$ & ${ }^{\mathrm{e}} /{ }^{\mathrm{e}}|171.0| 2.14 / 20.7$ & ---- & ---- & ${ }^{\mathrm{e}} /\left.\right|^{\mathrm{e}}|171.0| 2.14 / 20.7^{*}$ & ---- \\
\hline $\begin{array}{c}N^{1}-\mathrm{CHCOCH}_{3} \\
\mathrm{CH}_{3}\end{array}$ & ---- & $\begin{array}{c}6.95 / 75.4|168.5| \\
1.62 / 19.5 \\
2.10 / 20.7\end{array}$ & ---- & ---- & $\begin{array}{c}6.98 / 76.1 \mid 168.5 \\
1.62 / 19.5 \mid \\
2.11 / 20.7^{*}\end{array}$ \\
\hline$N^{3}-\mathrm{CD}_{2} \mathrm{OCOCH}_{3}$ & ---- & ---- & $---/^{b}|169.8| 2.09 / 20.7$ & ${ }^{\mathrm{e}} /{ }^{\mathrm{e}}|169.4| 2.08 / 20.7^{*}$ & ${ }^{\mathrm{e}} /{ }^{\mathrm{e}}|169.8| 2.08 / 20.7^{*}$ \\
\hline
\end{tabular}

${ }^{\mathrm{a} 1} \mathrm{H} /{ }^{13} \mathrm{C} /{ }^{15} \mathrm{~N}$ NMR chemical shifts given in a mixture $\mathbf{3 b D}$ and $\mathbf{3 c},{ }^{\mathrm{b}}{ }^{1} \mathrm{H} /{ }^{13} \mathrm{C} /{ }^{15} \mathrm{~N}$ NMR chemical shifts given in a mixture $\mathbf{3 f D}$ and $\mathbf{3 g D}$,

${ }^{\mathrm{c}}$ assignment could be reversed, ${ }^{\mathrm{d} 15} \mathrm{~N}$ chemical shifts not measured, ${ }^{\mathrm{e}}$ signals not observed in ${ }^{1} \mathrm{H}$ NMR and short accumulated ${ }^{13} \mathrm{C}$ NMR experiments, $*{ }^{13} \mathrm{C}$ signals of both methyl groups overlap 
Table S9. Crystal data and structure refinement for investigated compound

\begin{tabular}{|l|l|}
\hline Identification code & $\mathbf{2 9 6 - 5 5 - 3 - 2}$ \\
\hline Empirical formula & $\mathrm{C}_{8} \mathrm{H}_{10} \mathrm{~N}_{2} \mathrm{O}_{4}$ \\
\hline Formula weight & 198.18 \\
\hline Temperature/ K & $100(2)$ \\
\hline Crystal system & triclinic \\
\hline Space group & $P-1$ \\
\hline$a / \AA$ & $5.6131(4)$ \\
\hline$b / \AA$ & $8.2986(5)$ \\
\hline$c / \AA$ & $10.7345(6)$ \\
\hline$\alpha /{ }^{\circ}$ & $91.575(5)$ \\
\hline$\beta /^{\circ}$ & $103.325(5)$ \\
\hline$\gamma /{ }^{\circ}$ & $102.783(6)$ \\
\hline Volume/ $\AA^{3}$ & $472.88(5$ \\
\hline$Z$ & 2 \\
\hline$\rho_{\text {calc }}$ g/cm & \\
\hline$\mu /$ mm $^{-1}$ & 1.392 \\
\hline$F(000)$ & 0.968 \\
\hline Crystal size / mm & 208.0 \\
\hline Radiation & $0.27 \times 0.05 \times 0.04$ \\
\hline $2 \Theta$ range for data collection/ ${ }^{\circ}$ & $\mathrm{CuK}(\lambda=1.54184 \AA)$ \\
\hline Index ranges & 8.494 to 134.138 \\
\hline Reflections collected & $-6 \leq h \leq 6,-9 \leq k \leq 9,-12 \leq l \leq 12$ \\
\hline Independent reflections & 6195 \\
\hline Data/restraints/parameters & $1690\left[R_{\text {int }}=0.0269, R_{\text {sigma }}=0.0227\right]$ \\
\hline Goodness-of-fit on $F 2$ & $1690 / 1 / 132$ \\
\hline Final $R$ indexes $[I>=2 \sigma(I)]$ & 1.069 \\
\hline Final $R$ indexes [all data] & $R_{1}=0.0373, w R_{2}=0.0930$ \\
\hline Largest diff. peak/hole / e $\AA^{-3}$ & $R_{1}=0.0436, w R_{2}=0.0990$ \\
\hline & $0.19 /-0.28$ \\
\hline
\end{tabular}


Table S10. The geometry of the hydrogen bonds for the 296-55-3-2

\begin{tabular}{|c|c|c|c|c|}
\hline $\mathbf{D}-\mathbf{H} \cdots \mathbf{A}$ & D-H, [Å] & $\mathbf{H} \cdots \mathbf{A},[\AA ̊]$ & $\mathbf{D} \cdots \mathbf{A},[\AA ̊]$ & $(\mathrm{D}-\mathrm{H} \cdots \mathbf{A}),\left[^{\circ}\right]$ \\
\hline $\mathbf{N}(3)-H(3) \ldots O(13)^{i}$ & $0.865(17)$ & $2.003(17)$ & $2.8651(16)$ & $174.2(14)$ \\
\hline $\mathrm{C}(6)-\mathrm{H}(6) \ldots \mathrm{O}(12)^{\mathrm{ii}}$ & 0.93 & 2.30 & $3.220(2)$ & 171 \\
\hline $\mathrm{C}(7)-\mathrm{H}(7) \ldots \mathrm{O}(13)$ & 0.98 & 2.35 & $2.7760(19)$ & 105 \\
\hline $\mathrm{C}(7)-\mathrm{H}(7) \ldots \mathrm{O}(14){ }^{\mathrm{iii}}$ & 0.98 & 2.44 & $3.1534(18)$ & 129 \\
\hline $\mathrm{C}(11)-\mathrm{H}(11 \mathrm{~B}) \ldots \mathrm{O}(14)$ iv & 0.96 & 2.48 & $3.3974(19)$ & 160 \\
\hline
\end{tabular}

Symmetry codes for M3: ${ }^{\mathrm{i}}-\mathrm{x}, 1-\mathrm{y}, 2-\mathrm{z},{ }^{\mathrm{ii}} 1+\mathrm{x}, \mathrm{y}, \mathrm{z} ;{ }^{\mathrm{iii}} \mathrm{x}, 1+\mathrm{y}, \mathrm{z} ;{ }^{\mathrm{iv}} 1-\mathrm{x}, 1-\mathrm{y}, 2-\mathrm{z}$
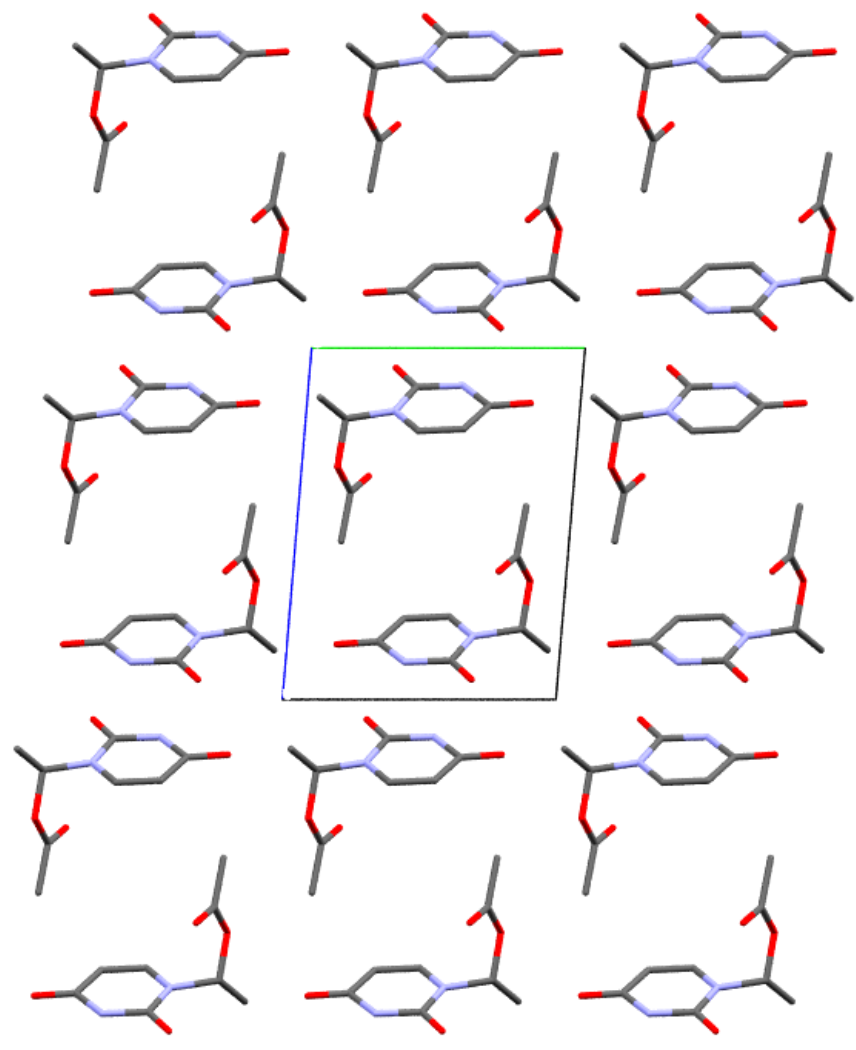

Figure S1. Supramolecular architecture of investigated compound in the crystal, viewed along $a$-direction. The $\mathrm{H}$ atoms were omitted for clarity. 


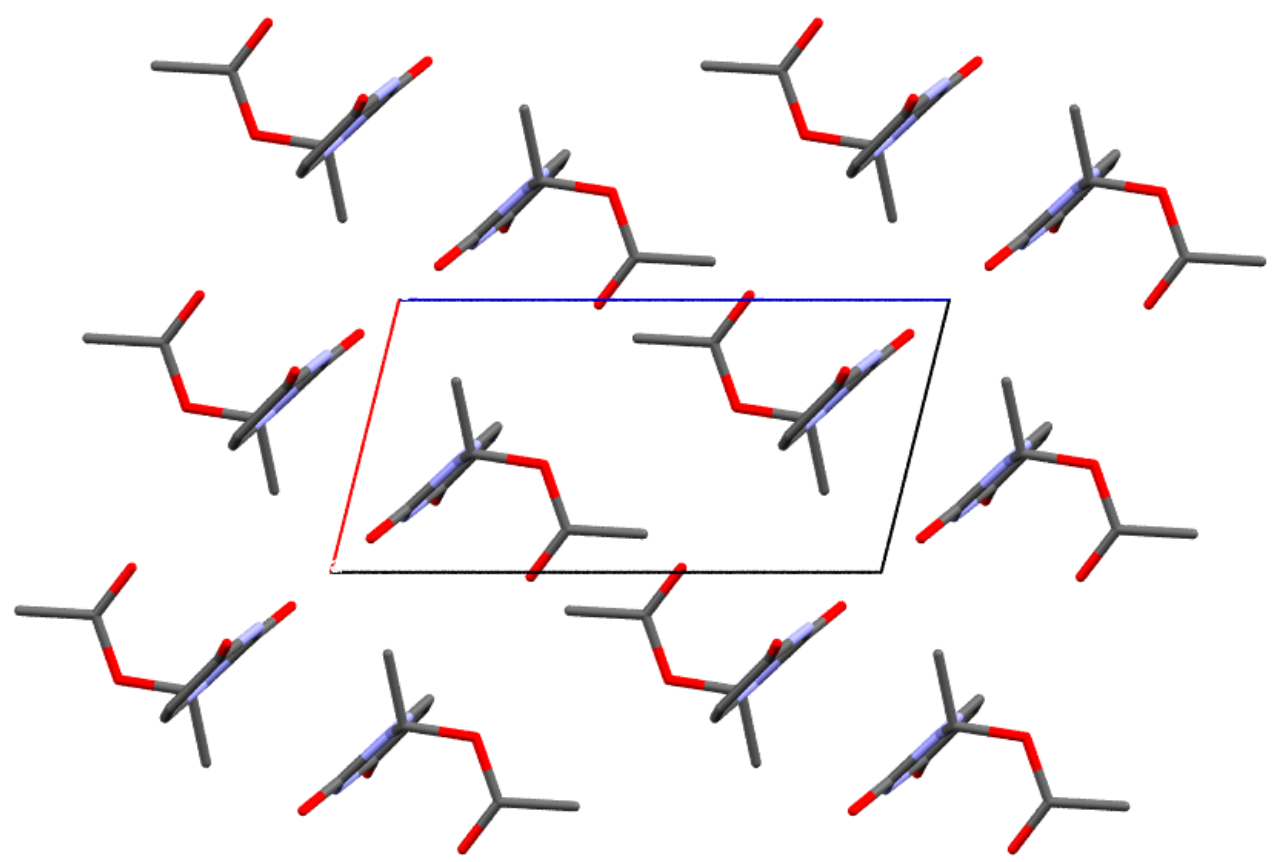

Figure S2. Supramolecular architecture of investigated compound in the crystal, viewed along $b$-direction. The $\mathrm{H}$ atoms were omitted for clarity.

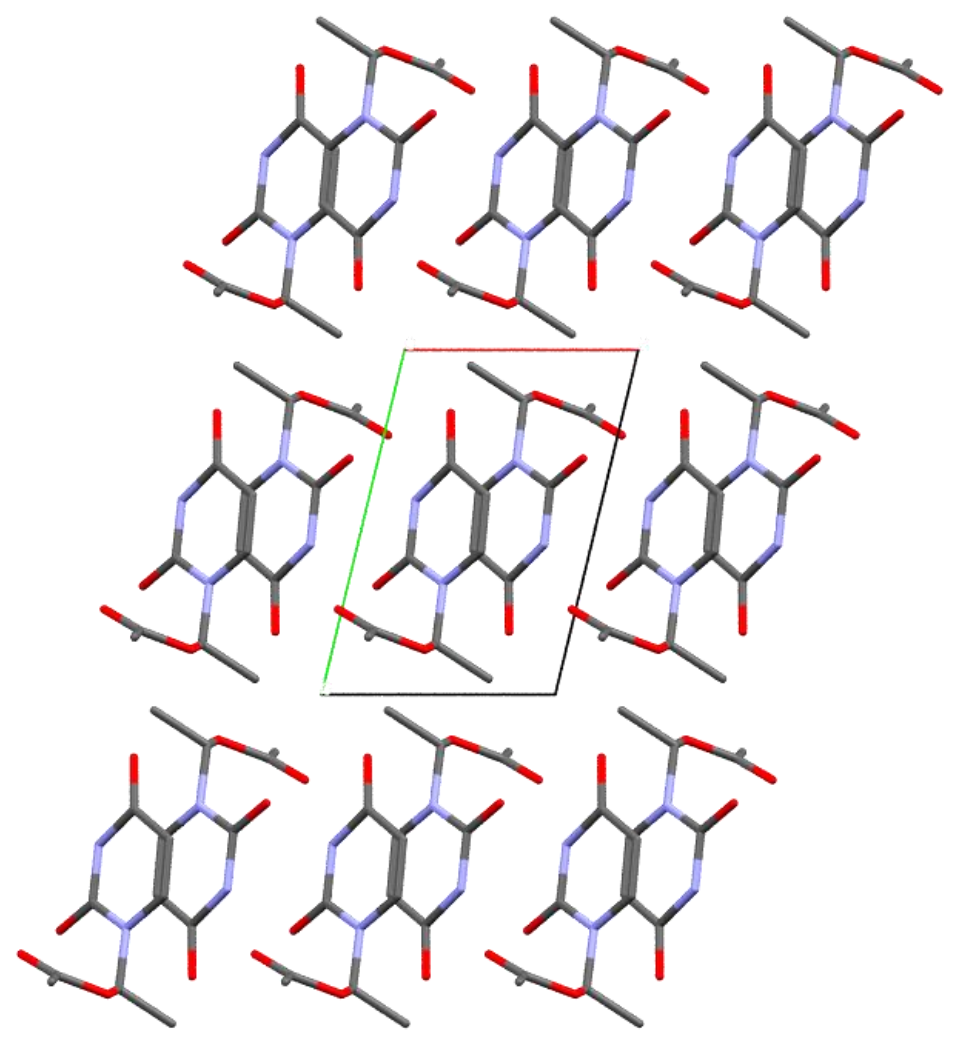

Figure S3. Supramolecular architecture of investigated compound in the crystal, viewed along $c$-direction. The $\mathrm{H}$ atoms were omitted for clarity. 
Table S11. Bond lengths for investigated compound

\begin{tabular}{|c|c|c|c|c|c|}
\hline Atom & Atom & Length/Å & Atom & Atom & Length/Å \\
\hline $\mathrm{C} 2$ & N1 & $1.3855(19)$ & C6 & N1 & $1.3753(19)$ \\
\hline $\mathrm{C} 2$ & N3 & $1.3742(19)$ & $\mathrm{C} 7$ & C11 & $1.503(2)$ \\
\hline $\mathrm{C} 2$ & O13 & $1.2262(18)$ & $\mathrm{C} 7$ & N1 & $1.4717(18)$ \\
\hline $\mathrm{C} 4$ & $\mathrm{C} 5$ & $1.446(2)$ & $\mathrm{C} 7$ & O8 & $1.4518(17)$ \\
\hline $\mathrm{C} 4$ & N3 & $1.3936(19)$ & C9 & $\mathrm{C} 10$ & $1.500(2)$ \\
\hline $\mathrm{C} 4$ & O14 & $1.2226(18)$ & C9 & O8 & $1.3483(19)$ \\
\hline $\mathrm{C} 5$ & C6 & $1.338(2)$ & C9 & 012 & 1.201(2) \\
\hline
\end{tabular}

Table S12. Valence angles for investigated compound.

\begin{tabular}{|l|l|l|l|l|l|l|l|}
\hline Atom & Atom & Atom & Angle $^{\circ}$ & Atom & Atom & Atom & Angle $^{\circ}$ \\
\hline N3 & C2 & N1 & $114.99(12)$ & O8 & C7 & N1 & $107.96(11)$ \\
\hline O13 & C2 & N1 & $122.69(13)$ & O8 & C9 & C10 & $111.36(14)$ \\
\hline O13 & C2 & N3 & $122.32(13)$ & O12 & C9 & C10 & $125.08(16)$ \\
\hline N3 & C4 & C5 & $113.75(12)$ & O12 & C9 & O8 & $123.53(14)$ \\
\hline O14 & C4 & C5 & $125.83(13)$ & C2 & N1 & C7 & $118.99(12)$ \\
\hline O14 & C4 & N3 & $120.42(13)$ & C6 & N1 & C2 & $121.30(12)$ \\
\hline C6 & C5 & C4 & $120.13(13)$ & C6 & N1 & C7 & $119.56(12)$ \\
\hline C5 & C6 & N1 & $122.58(13)$ & C2 & N3 & C4 & $127.18(12)$ \\
\hline N1 & C7 & C11 & $112.59(12)$ & C9 & O8 & C7 & $116.88(11)$ \\
\hline
\end{tabular}

Table S13. Torsion angles for investigated compound

\begin{tabular}{|l|l|l|l|l|l|l|l|l|l|}
\hline $\mathbf{A}$ & $\mathbf{B}$ & $\mathbf{C}$ & $\mathbf{D}$ & $\mathbf{A n g l e}^{\circ}$ & $\mathbf{A}$ & $\mathbf{B}$ & $\mathbf{C}$ & $\mathbf{D}$ & Angle $^{\circ}$ \\
\hline C4 & C5 & C6 & N1 & $0.5(2)$ & N3 & C2 & N1 & C7 & $174.15(12)$ \\
\hline C5 & C4 & N3 & C2 & $2.8(2)$ & N3 & C4 & C5 & C6 & $-2.5(2)$ \\
\hline C5 & C6 & N1 & C2 & $1.6(2)$ & O8 & C7 & N1 & C2 & $-124.78(13)$ \\
\hline C5 & C6 & N1 & C7 & $-173.89(14)$ & O8 & C7 & N1 & C6 & $50.81(16)$ \\
\hline C10 & C9 & O8 & C7 & $-178.51(14)$ & O12 & C9 & O8 & C7 & $-0.1(2)$ \\
\hline C11 & C7 & N1 & C2 & $117.92(14)$ & O13 & C2 & N1 & C6 & $177.96(13)$ \\
\hline C11 & C7 & N1 & C6 & $-66.50(16)$ & O13 & C2 & N1 & C7 & $-6.5(2)$ \\
\hline C11 & C7 & O8 & C9 & $-168.83(12)$ & O13 & C2 & N3 & C4 & $179.70(13)$ \\
\hline N1 & C2 & N3 & C4 & $-1.0(2)$ & O14 & C4 & C5 & C6 & $177.29(14)$ \\
\hline N1 & C7 & O8 & C9 & $70.02(15)$ & O14 & C4 & N3 & C2 & $-176.95(13)$ \\
\hline N3 C2 N1 & C6 & & $-1.35(19)$ & & & & & \\
\hline
\end{tabular}


Table S14. Coordinates Atom Positions

\begin{tabular}{|c|c|c|c|}
\hline Atom & $\mathbf{x}$ & $\mathbf{y}$ & $\mathbf{Z}$ \\
\hline $\mathrm{O} 8$ & $0.39472(19)$ & $0.86950(12)$ & $0.66203(10)$ \\
\hline O12 & $-0.0169(2)$ & $0.75269(17)$ & $0.63518(11)$ \\
\hline O13 & $0.12438(19)$ & $0.68499(12)$ & $0.94311(10)$ \\
\hline O14 & $0.2626(2)$ & $0.18266(12)$ & $0.84304(10)$ \\
\hline N1 & $0.4065(2)$ & $0.67675(14)$ & $0.82022(11)$ \\
\hline N3 & $0.2024(2)$ & $0.43592(14)$ & $0.89358(11)$ \\
\hline $\mathrm{C} 2$ & $0.2358(3)$ & $0.60458(17)$ & $0.88907(13)$ \\
\hline $\mathrm{C} 4$ & $0.3175(3)$ & $0.33251(18)$ & $0.83567(13)$ \\
\hline $\mathrm{C} 5$ & $0.4981(3)$ & $0.41968(18)$ & $0.76954(14)$ \\
\hline C6 & $0.5343(3)$ & $0.58389(18)$ & $0.76399(14)$ \\
\hline $\mathrm{C} 7$ & $0.4370(3)$ & $0.85393(17)$ & $0.79932(14)$ \\
\hline C9 & $0.1568(3)$ & $0.8135(2)$ & $0.59087(15)$ \\
\hline $\mathrm{C} 10$ & $0.1409(4)$ & $0.8330(3)$ & $0.45089(17)$ \\
\hline $\mathrm{C} 11$ & $0.6988(3)$ & $0.95408(18)$ & $0.85917(15)$ \\
\hline $\mathrm{H} 3$ & $0.100(3)$ & $0.393(2)$ & $0.9394(15)$ \\
\hline H5 & 0.58930 & 0.36150 & 0.73080 \\
\hline H6 & 0.65040 & 0.63740 & 0.72040 \\
\hline $\mathrm{H} 7$ & 0.31440 & 0.89840 & 0.83310 \\
\hline H10A & 0.25710 & 0.93310 & 0.44140 \\
\hline H10B & -0.02710 & 0.83840 & 0.40830 \\
\hline $\mathrm{H} 10 \mathrm{C}$ & 0.18270 & 0.73980 & 0.41330 \\
\hline H11A & 0.81680 & 0.91520 & 0.82110 \\
\hline H11B & 0.73880 & 0.94220 & 0.94980 \\
\hline $\mathrm{H} 11 \mathrm{C}$ & 0.70810 & 1.06870 & 0.84490 \\
\hline
\end{tabular}

Supplementary crystallographic data for this paper have been deposited at the Cambridge Crystallographic Data Centre (CCDC). This data can be obtained freely via http://www.ccdc.cam.ac.uk/data_request/cif, by e-mailing data_request@ccdc.cam.ac.uk or by contacting directly the Cambridge Crystallographic Data Centre (12 Union Road, Cambridge CB2 1EZ, UK. Fax: +44 1223 336033). 
Table S15. Yield of selected reactions with $\mathrm{Ac}_{2} \mathrm{O}$.

\begin{tabular}{|c|c|c|c|c|c|c|c|}
\hline & $A^{a}$ & $\mathrm{B1}^{\mathrm{b}}$ & $\mathrm{B} 2^{\mathrm{b}}$ & $\mathrm{C}^{\mathrm{c}}$ & $D^{d}$ & $\mathrm{E1}^{\mathrm{e}}$ & E2 ${ }^{\mathrm{e}}$ \\
\hline Product & 1.5 eq. $\mathrm{Ac}_{2} \mathrm{O}$ & 1.5 eq. $\mathrm{Ac}_{2} \mathrm{O}$ & 3 eq. $\mathrm{Ac}_{2} \mathrm{O}$ & 1.5 eq. $\mathrm{Ac}_{2} \mathrm{O}$ & 1.5 eq. $\mathrm{Ac}_{2} \mathrm{O}$ & 1.5 eq. $\mathrm{Ac}_{2} \mathrm{O}$ & 3 eq. $A c_{2} \mathrm{O}$ \\
\hline $1 \mathrm{~b}$ & - & 6 & 4 & 9 & - & - & - \\
\hline $1 e$ & & 1 & 1 & 9 & - & - & - \\
\hline 1f & - & $8^{j}$ & 10 & 3 & - & - & - \\
\hline $2 \mathbf{a}$ & 69 & - & - & 45 & - & - & - \\
\hline $2 b$ & 一 & 6 & 8 & 2 & 6 & - & - \\
\hline $2 c$ & - & 4 & 3 & $<1$ & 1 & 3 & 9 \\
\hline $2 b: 2 c$ & & $57: 43^{k}$ & $72: 28$ & & & & \\
\hline $2 e$ & - & 18 & 19 & 3.4 & 17 & - & - \\
\hline $2 f$ & - & 10 & 30 & - & 12 & - & - \\
\hline $2 g$ & - & 3 & 2 & - & 2 & - & - \\
\hline $2 f: 2 g$ & - & $78: 22$ & $93: 7$ & - & - & - & - \\
\hline $2 \mathbf{i}$ & - & - & - & - & - & 3 & 1 \\
\hline $2 \mathbf{j}$ & - & - & - & - & - & 9 & 4 \\
\hline 21 & - & - & - & - & - & 2 & 6 \\
\hline $3 \mathbf{a}$ & 67 & - & - & - & - & - & - \\
\hline $3 \mathbf{b}$ & - & 5 & 4 & 1 & - & - & - \\
\hline $3 c$ & - & 2 & 3 & 1 & - & 8 & 8 \\
\hline $3 b: 3 c$ & - & $71: 29$ & $56: 44$ & - & - & - & - \\
\hline $3 e$ & - & 3 & 11 & - & - & - & - \\
\hline $3 f$ & - & 3 & 3 & 2.9 & - & - & - \\
\hline $3 g$ & - & $<1$ & 1 & 1 & - & $<1$ & 2 \\
\hline 3f:3g & - & $95: 5$ & 81:19 & - & - & - & - \\
\hline $3 \mathbf{i}$ & - & - & - & - & - & 3 & 3 \\
\hline $3 \mathbf{j}$ & - & - & - & - & - & 7 & 9 \\
\hline 31 & - & - & - & - & - & 9 & 8 \\
\hline $3 n$ & - & - & - & - & - & 2 & 3 \\
\hline $3 m$ & - & - & - & - & - & 1 & $<1$ \\
\hline
\end{tabular}

a) Procedure $\mathrm{A}: \mathrm{Ac}_{2} \mathrm{O}$, pyridine, $\mathrm{ACN}, 55^{\circ} \mathrm{C}$

b) Procedure B: $\mathrm{Ac}_{2} \mathrm{O}$, TEA, DMAP, DCM, r.t

c) Procedure C: $\mathrm{Ac}_{2} \mathrm{O}$, TEA, DCM, r.t.

d) Procedure D: $\mathrm{Ac}_{2} \mathrm{O}$, TEA, DMAP-D6, DCM, r.t

e) Procedure E: $\mathrm{Ac}_{2} \mathrm{O}$, TEA, DMAP, 1,2-dichloroetan, r.t

j) yield

k) On the basis of the NMR spectra the percentage content ratio of each compound in the mixture was calculated 
Table S16. Yield of selected reactions with $\left(\mathrm{C}_{2} \mathrm{H}_{5} \mathrm{OC}\right)_{2} \mathrm{O}$ and with $\mathrm{Ac}_{2} \mathrm{O}$ in $\mathrm{CD}_{2} \mathrm{Cl}_{2}$.

\begin{tabular}{|c|c|c|c|c|c|}
\hline & $\mathrm{F}^{\mathrm{f}}$ & $\mathrm{G}^{\mathrm{g}}$ & $\mathrm{H}^{\mathrm{h}}$ & & $\mathrm{I}^{\mathrm{i}}$ \\
\hline Product & 1.5eq $\left(\mathrm{C}_{2} \mathrm{H}_{5} \mathrm{OC}\right)_{2} \mathrm{O}$ & 1.5eq $\left(\mathrm{C}_{2} \mathrm{H}_{5} \mathrm{OC}\right)_{2} \mathrm{O}$ & 1.5 eq. $\mathrm{Ac}_{2} \mathrm{O}$ & Product & 1.5eq. $\mathrm{Ac}_{2} \mathrm{O}$ \\
\hline $2 b$ & & & 5 & $2 \mathrm{bD}$ & 15 \\
\hline $2 b 1$ & 5 & $4^{j}$ & & $2 c$ & 1 \\
\hline $2 c$ & & & $<1$ & $2 \mathrm{eD}$ & 11 \\
\hline $2 \mathrm{c1}$ & 4 & 1 & & $2 \mathrm{fD}$ & 4 \\
\hline $2 d$ & & & 10 & $2 \mathrm{gD}$ & - \\
\hline 2d1 & & 26 & & & \\
\hline $2 e$ & & & 3 & & \\
\hline $2 \mathrm{e} 1$ & 5 & & & 3bD & 10 \\
\hline $2 f$ & & & 1 & $3 c$ & 11 \\
\hline $2 \mathrm{f1}$ & 5 & $<1$ & & $3 \mathrm{eD}$ & 16 \\
\hline $2 g$ & & & & 3fD & 5 \\
\hline $2 \mathrm{~g} 1$ & 2 & $<1$ & & 3gD & 2 \\
\hline $2 \mathrm{~h}$ & & & 4 & & \\
\hline $2 \mathrm{~h} 1$ & & 6 & & & \\
\hline
\end{tabular}

f) Procedure F: (C2H5OC)2O, TEA, DMAP, DCM, r.t

g) Procedue G: (C2H5OC)2O, TPA, DMAP, DCM, r.t

h) Procedure H: Ac2O, TPA, DMAP, DCM, r.t

i) Procedure I: Ac2O, TEA, DMAP, CD2Cl2, r.t

j) yield 


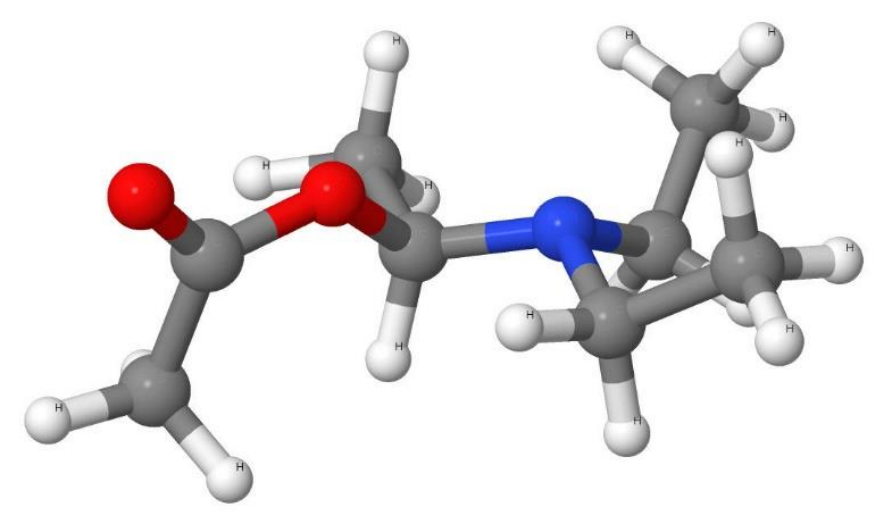

Figure S4. The acetyloxydiethylamine ( $N^{1}$-acetyloxyethyl diethylamine) appearing in the Step3, Scheme 4 of the proposed mechanism of acetylation (see the text).

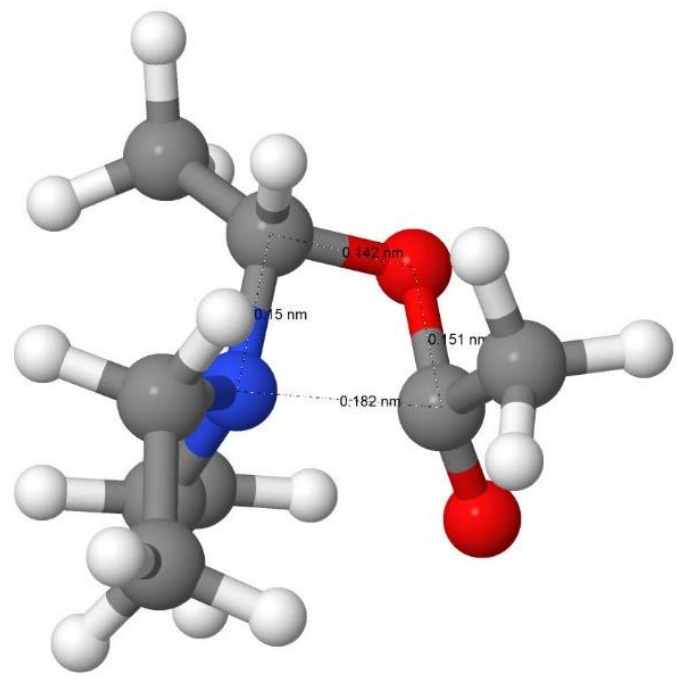

Figure S5. The transition state species with four-member ring $\mathrm{N}-\mathrm{C}-\mathrm{O}-\mathrm{C}$ appearing in the Step3, Scheme 4 of the proposed mechanism of acetylation (see the text). 


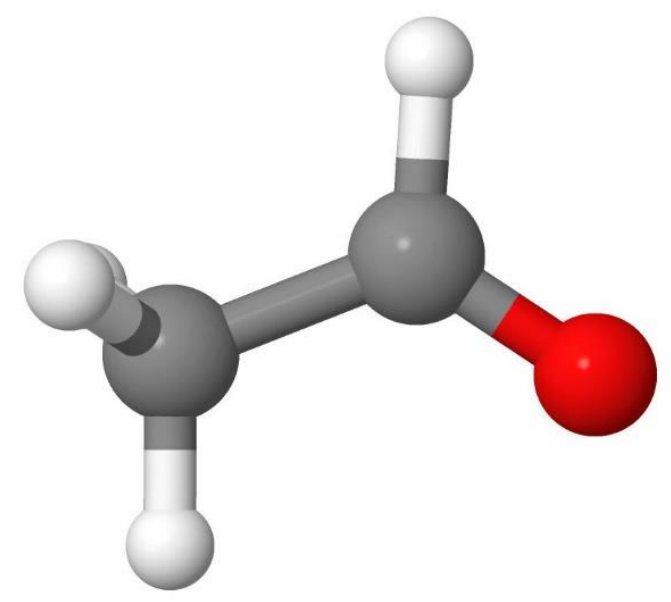

Figure S6. The acetaldehyde (III) appearing in the Step3, Scheme 4 of the proposed mechanism of acetylation (see the text).

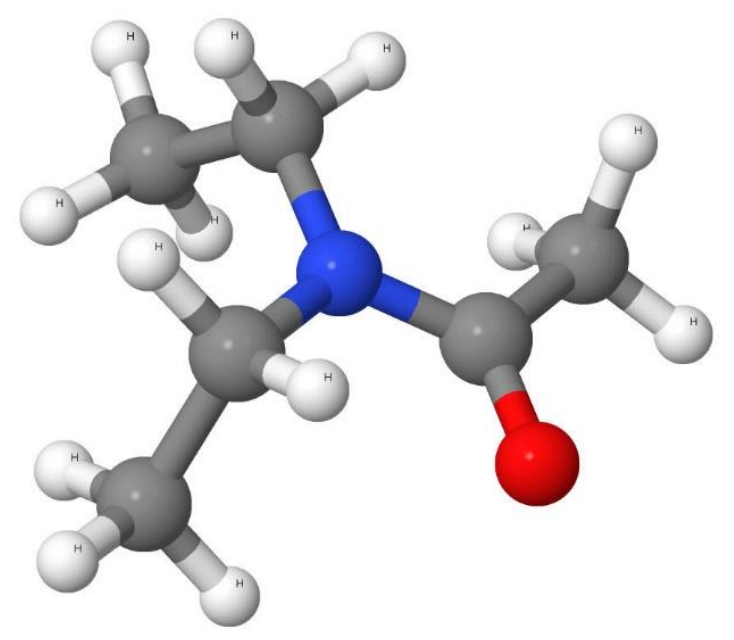

Figure S7. The acetyldiethylamine (IV) appearing in the Step3, Scheme 4 of the proposed mechanism of acetylation (see the text). 
Table S17. Reaction, uphill and downhill transition states as well as Eq. 2 details (Filenames correspond to the Cartesian coordinate files in the Table S18).

\section{Reaction details}

\begin{tabular}{|c|c|c|c|}
\hline File name & Code & Gibbs free energy & Comments \\
\hline $\mathrm{UCH}_{2} \mathrm{Cl}_{2}$ & L1 & -414.913216 & Uracil \\
\hline $\mathrm{Ac}_{2} \mathrm{Odichl}$ & $\mathrm{L} 2$ & -381.797307 & $\mathrm{Ac}_{2} \mathrm{O}$ \\
\hline TEA & L3 & -292.338634 & Triethylamine \\
\hline $\mathrm{CH}_{2} \mathrm{Cl}_{2} \mathrm{opt}$ & $\mathrm{L} 4$ & -959.773484 & $\mathrm{CH}_{2} \mathrm{Cl}_{2}$ \\
\hline DMAPppb & L5 & -382.249408 & DMAP \\
\hline Path1h & $\mathrm{R} 1$ & -721.411514 & Product 3c \\
\hline $\mathrm{NEt}_{2} \mathrm{Ac}$ & $\mathrm{R} 2$ & -366.432957 & $N$-Ac-diethylamine \\
\hline $\mathrm{CH}_{3} \mathrm{Cl}$ & R3 & -500.143621 & $\mathrm{CH}_{3} \mathrm{Cl}$ \\
\hline DMAPAcCl & $\mathrm{R} 4$ & -995.7714 & {$\left[N^{1}-\mathrm{Ac}-\mathrm{DMAP}\right]^{(+)} \mathrm{Cl}^{(-)}$} \\
\hline DMAPAA & R5 & -611.375324 & $\mathrm{DMAP} \times \mathrm{CH}_{3} \mathrm{COOH}$ \\
\hline
\end{tabular}

\section{Uphill Transition state details}

\begin{tabular}{cccc}
\hline File name & Code & Gibbs free energy & Comments \\
\hline Path1c & L1 & -520.249865 & $\mathrm{Et}_{2}-\mathrm{N}-\mathrm{CH}\left(\mathrm{CH}_{3}\right)-\mathrm{O}-\mathrm{Ac}$ \\
Path1dccF & R1 & -520.206889 & Transition state \\
\hline
\end{tabular}

Downhill Transition state details

\begin{tabular}{cccc}
\hline File name & Code & Gibbs free energy & Comments \\
\hline Path1dccF & L1 & -520.206889 & Transition state \\
& & & \\
AcH & R1 & -153.85989 & Acetaldehyde \\
$\mathrm{NEt}_{2} \mathrm{Ac}$ & $\mathrm{R} 2$ & -366.432957 & $N-$ Ac-diethylamine \\
\hline
\end{tabular}

Eq.2 details

\begin{tabular}{cccc}
\hline Filename & Code & Gibbs free Energy (Hartree) & Comments \\
\hline $\mathrm{UCH}_{2} \mathrm{Cl}_{2}$ & L1 & -414.913216 & Uracil \\
TEA & L2 & -292.338634 & Triethylamine \\
$\mathrm{CH}_{2} \mathrm{Cl}_{2} \mathrm{Opt}$ & L3 & -959.773484 & $\mathrm{CH}_{2} \mathrm{Cl}_{2}$ \\
$\mathrm{Ac}_{2}$ Odichl & L4 & -381.797307 & $\mathrm{Ac}_{2} \mathrm{O}$ \\
& & & \\
Path2prodN1 & $\mathrm{R} 1$ & -682.121234 & Product $\mathbf{3 b}$ \\
TEAHCl & $\mathrm{R} 2$ & -753.215411 & Triethylamine $\times \mathrm{HCl}$ \\
AcCldichl & $\mathrm{R} 3$ & -613.515515 & Acetyl chloride \\
\hline
\end{tabular}


Table S18. The Cartesian coordinates $[\AA]$.

\section{Structure $\mathrm{Ac}_{2}$ Odichl}

\begin{tabular}{lrrrr} 
& Atom & $x$ & $y$ & $z$ \\
\hline 1 & $\mathrm{C}$ & -0.0222 & -0.1091 & 0.0006 \\
2 & $\mathrm{C}$ & 1.1697 & -1.0089 & 0.0647 \\
3 & $\mathrm{O}$ & 2.3444 & -0.2583 & 0.0614 \\
4 & $\mathrm{C}$ & 3.5489 & -0.7640 & -0.4253 \\
5 & $\mathrm{C}$ & 4.7035 & -0.0908 & 0.2440 \\
6 & $\mathrm{H}$ & 4.5758 & 0.9939 & 0.2189 \\
7 & $\mathrm{H}$ & 4.7311 & -0.3944 & 1.2951 \\
8 & $\mathrm{H}$ & 5.6335 & -0.3737 & -0.2456 \\
9 & $\mathrm{O}$ & 3.5932 & -1.5901 & -1.2918 \\
10 & $\mathrm{O}$ & 1.1732 & -2.2035 & 0.1537 \\
11 & $\mathrm{H}$ & -0.0538 & 0.3690 & -0.9833 \\
12 & $\mathrm{H}$ & -0.9318 & -0.6851 & 0.1596 \\
13 & $\mathrm{H}$ & 0.0611 & 0.6833 & 0.7481
\end{tabular}

\section{Structure AcCldichl}

\begin{tabular}{rrrrr} 
& Atom & $x$ & $y$ & $z$ \\
\hline 1 & $\mathrm{C}$ & -0.0686 & 0.0167 & 0.0203 \\
2 & $\mathrm{C}$ & 0.9230 & 1.1247 & 0.1073 \\
3 & $\mathrm{C} 1$ & 2.5320 & 0.6832 & -0.7132 \\
4 & 0 & 0.8230 & 2.1914 & 0.6045 \\
5 & $\mathrm{H}$ & 0.3413 & -0.8767 & 0.4974 \\
6 & $\mathrm{H}$ & -0.9914 & 0.3224 & 0.5131 \\
7 & $\mathrm{H}$ & -0.2569 & -0.2213 & -1.0293
\end{tabular}

\section{Structure AcH}

\begin{tabular}{rrrrr} 
& Atom & $x$ & $y$ & $z$ \\
\hline 1 & $\mathrm{C}$ & -0.3971 & 0.0700 & 0.6034 \\
2 & $\mathrm{O}$ & -2.5843 & -0.8656 & 0.2492 \\
3 & $\mathrm{C}$ & -1.7142 & -0.0880 & -0.0840 \\
4 & $\mathrm{H}$ & -0.3021 & -0.6037 & 1.4559 \\
5 & $\mathrm{H}$ & -0.2886 & 1.1116 & 0.9282 \\
6 & $\mathrm{H}$ & 0.4065 & -0.1160 & -0.1188 \\
7 & $\mathrm{H}$ & -1.8721 & 0.5726 & -0.9601
\end{tabular}

\section{Structure $\mathrm{CH}_{\mathbf{2}} \mathrm{Cl}_{2} \mathrm{opt}$}

\begin{tabular}{rrrrr} 
& Atom & $x$ & $y$ & $z$ \\
\hline 1 & $\mathrm{C}$ & -0.0015 & -0.0026 & 0.0021 \\
2 & $\mathrm{H}$ & 0.0131 & 0.0227 & 1.0858 \\
3 & $\mathrm{Cl}$ & 1.7002 & -0.0440 & -0.5742 \\
4 & $\mathrm{H}$ & -0.5075 & -0.8790 & -0.3867 \\
5 & $\mathrm{Cl}$ & -0.8882 & 1.4505 & -0.5742
\end{tabular}

\section{Structure $\mathrm{CH}_{3} \mathrm{Cl}$}

\begin{tabular}{rrrrr} 
& Atom & $x$ & $y$ & $z$ \\
\hline 1 & $\mathrm{C}$ & -0.0931 & -0.0424 & 0.0000 \\
2 & $\mathrm{Cl}$ & 1.7233 & -0.1181 & 0.0000 \\
3 & $\mathrm{H}$ & -0.3833 & 1.0051 & 0.0000 \\
4 & $\mathrm{H}$ & -0.4471 & -0.5456 & 0.8961 \\
5 & $\mathrm{H}$ & -0.4471 & -0.5456 & -0.8961
\end{tabular}


Table S18. continuation.

\section{Structure DMAPAA}

\begin{tabular}{lrrrr}
\multicolumn{1}{r}{ Atom } & $\mathrm{x}$ & $\mathrm{y}$ \\
\hline 1 & $\mathrm{~N}$ & -0.3919 & -0.5377 & -0.7543 \\
2 & $\mathrm{C}$ & -0.3038 & -1.8333 & -0.2265 \\
3 & $\mathrm{C}$ & -0.8328 & -2.1608 & 1.0376 \\
4 & $\mathrm{C}$ & -0.6657 & -3.4450 & 1.5373 \\
5 & $\mathrm{~N}$ & -0.0304 & -4.4342 & 0.8937 \\
6 & $\mathrm{C}$ & 0.4630 & -4.1229 & -0.3131 \\
7 & $\mathrm{C}$ & 0.3558 & -2.8740 & -0.9096 \\
8 & $\mathrm{H}$ & 0.7871 & -2.7290 & -1.8895 \\
9 & $\mathrm{H}$ & 0.9748 & -4.9205 & -0.8445 \\
10 & $\mathrm{H}$ & -1.0739 & -3.6900 & 2.5140 \\
11 & $\mathrm{H}$ & -1.3663 & -1.4373 & 1.6368 \\
12 & $\mathrm{C}$ & -1.4456 & 0.3364 & -0.2129 \\
13 & $\mathrm{H}$ & -1.3575 & 1.3141 & -0.6855 \\
14 & $\mathrm{H}$ & -1.3241 & 0.4746 & 0.8610 \\
15 & $\mathrm{H}$ & -2.4486 & -0.0631 & -0.4100 \\
16 & $\mathrm{C}$ & -0.2107 & -0.3916 & -2.2078 \\
17 & $\mathrm{H}$ & -0.2615 & 0.6683 & -2.4549 \\
18 & $\mathrm{H}$ & -0.9879 & -0.9242 & -2.7706 \\
19 & $\mathrm{H}$ & 0.7688 & -0.7555 & -2.5133 \\
20 & $\mathrm{H}$ & 1.1498 & 0.3260 & -0.0480 \\
21 & $\mathrm{C}$ & 4.0642 & 1.5880 & 0.5705 \\
22 & $\mathrm{C}$ & 3.0153 & 0.7935 & -0.1543 \\
23 & $\mathrm{O}$ & 3.2030 & 0.1481 & -1.1660 \\
24 & $\mathrm{O}$ & 1.8167 & 0.8698 & 0.4457 \\
25 & $\mathrm{H}$ & 5.0148 & 1.5208 & 0.0449 \\
26 & $\mathrm{H}$ & 3.7531 & 2.6333 & 0.6452 \\
27 & $\mathrm{H}$ & 4.1766 & 1.2054 & 1.5886
\end{tabular}

\section{Structure DMAPAcCI}

\begin{tabular}{lrrrr} 
& Atom & $x$ & $y$ & $z$ \\
\hline 1 & $\mathrm{C}$ & -0.0102 & 0.2256 & 0.9212 \\
2 & $\mathrm{~N}$ & 0.5712 & -1.1115 & 0.7408 \\
3 & $\mathrm{C}$ & 1.8777 & -1.3146 & 0.9185 \\
4 & $\mathrm{C}$ & 2.7671 & -0.2586 & 1.2882 \\
5 & $\mathrm{C}$ & 4.0950 & -0.4919 & 1.4633 \\
6 & $\mathrm{~N}$ & 4.6340 & -1.7438 & 1.2933 \\
7 & $\mathrm{C}$ & 3.8066 & -2.7807 & 0.9381 \\
8 & $\mathrm{C}$ & 2.4740 & -2.6063 & 0.7500 \\
9 & $\mathrm{H}$ & 1.8830 & -3.4656 & 0.4715 \\
10 & $\mathrm{H}$ & 4.2887 & -3.7394 & 0.8196 \\
11 & $\mathrm{C}$ & 6.0552 & -2.0257 & 1.4740 \\
12 & $\mathrm{C}$ & 6.9328 & -0.8791 & 1.8606 \\
13 & $\mathrm{H}$ & 6.9009 & -0.0885 & 1.1065 \\
14 & $\mathrm{H}$ & 7.9506 & -1.2521 & 1.9544 \\
15 & $\mathrm{H}$ & 6.6138 & -0.4436 & 2.8111 \\
16 & $\mathrm{O}$ & 6.4364 & -3.1555 & 1.3029 \\
17 & $\mathrm{C} 1$ & 5.0083 & 2.8559 & 2.3146 \\
18 & $\mathrm{H}$ & 4.7545 & 0.3210 & 1.7436 \\
19 & $\mathrm{H}$ & 2.4155 & 0.7507 & 1.4392 \\
20 & $\mathrm{C}$ & -0.3153 & -2.2185 & 0.3612 \\
21 & $\mathrm{H}$ & -1.3283 & -1.8373 & 0.2702
\end{tabular}


Table S18. continuation.

$\begin{array}{rrrrr}22 & \mathrm{H} & -0.3043 & -3.0038 & 1.1215 \\ 23 & \mathrm{H} & -0.0149 & -2.6456 & -0.5992 \\ 24 & \mathrm{H} & -1.0783 & 0.1697 & 0.7296 \\ 25 & \mathrm{H} & 0.4342 & 0.9394 & 0.2235 \\ 26 & \mathrm{H} & 0.1445 & 0.5815 & 1.9427\end{array}$

\section{Structure DMAPppb}

\begin{tabular}{lrrrr} 
& Atom & $\mathrm{x}$ & $\mathrm{y}$ & $\mathrm{z}$ \\
\hline 1 & $\mathrm{~N}$ & -1.5483 & 0.0007 & -0.0906 \\
2 & $\mathrm{C}$ & -0.1828 & -0.0013 & -0.0458 \\
3 & $\mathrm{C}$ & 0.5730 & 1.1976 & -0.0305 \\
4 & $\mathrm{C}$ & 0.5733 & -1.1997 & -0.0164 \\
5 & $\mathrm{H}$ & 0.0935 & 2.1663 & -0.0358 \\
6 & $\mathrm{H}$ & 0.0947 & -2.1689 & -0.0063 \\
7 & $\mathrm{~N}$ & 2.6741 & -0.0005 & 0.0061 \\
8 & $\mathrm{C}$ & 1.9571 & -1.1362 & 0.0044 \\
9 & $\mathrm{C}$ & 1.9569 & 1.1349 & -0.0097 \\
10 & $\mathrm{H}$ & 2.5267 & -2.0618 & 0.0254 \\
11 & $\mathrm{H}$ & 2.5263 & 2.0608 & -0.0022 \\
12 & $\mathrm{C}$ & -2.2807 & 1.2538 & 0.0631 \\
13 & $\mathrm{C}$ & -2.2886 & -1.2505 & 0.0316 \\
14 & $\mathrm{H}$ & -3.3453 & 1.0540 & -0.0438 \\
15 & $\mathrm{H}$ & -3.3427 & -1.0554 & -0.1588 \\
16 & $\mathrm{H}$ & -2.1920 & -1.6944 & 1.0313 \\
17 & $\mathrm{H}$ & -1.9466 & -1.9810 & -0.7054 \\
18 & $\mathrm{H}$ & -1.9946 & 1.9748 & -0.7075 \\
19 & $\mathrm{H}$ & -2.1125 & 1.7119 & 1.0462
\end{tabular}

\section{Structure $\mathrm{NEt}_{2} \mathrm{Ac}$}

\begin{tabular}{lrrrr} 
& Atom & $\mathrm{x}$ & $\mathrm{y}$ & $\mathrm{z}$ \\
\hline 1 & $\mathrm{C}$ & -0.7771 & 0.1586 & -0.9661 \\
2 & $\mathrm{C}$ & -1.9121 & 0.5836 & -0.0321 \\
3 & $\mathrm{C}$ & 1.2858 & -0.7783 & 0.0901 \\
4 & $\mathrm{C}$ & 0.6602 & -2.1605 & 0.0368 \\
5 & $\mathrm{C}$ & 1.1762 & 1.6163 & -0.3311 \\
6 & $\mathrm{C}$ & 1.1172 & 2.2882 & 1.0424 \\
7 & $\mathrm{~N}$ & 0.5649 & 0.2777 & -0.3792 \\
8 & $\mathrm{O}$ & 2.4270 & -0.6281 & 0.5429 \\
9 & $\mathrm{H}$ & -2.8741 & 0.4406 & -0.5340 \\
10 & $\mathrm{H}$ & -1.8322 & 1.6374 & 0.2451 \\
11 & $\mathrm{H}$ & -1.9136 & -0.0127 & 0.8847 \\
12 & $\mathrm{H}$ & 0.5566 & -2.5045 & -0.9967 \\
13 & $\mathrm{H}$ & -0.3290 & -2.1868 & 0.4993 \\
14 & $\mathrm{H}$ & 1.3193 & -2.8469 & 0.5664 \\
15 & $\mathrm{H}$ & 0.6581 & 2.2296 & -1.0714 \\
16 & $\mathrm{H}$ & 2.2174 & 1.5238 & -0.6468 \\
17 & $\mathrm{H}$ & 1.5964 & 3.2711 & 0.9914 \\
18 & $\mathrm{H}$ & 1.6455 & 1.6933 & 1.7902 \\
19 & $\mathrm{H}$ & 0.0865 & 2.4310 & 1.3758 \\
20 & $\mathrm{H}$ & -0.9303 & -0.8692 & -1.2895 \\
21 & $\mathrm{H}$ & -0.7938 & 0.7751 & -1.8700
\end{tabular}


Table S18. continuation.

\section{Structure Path1c}

\begin{tabular}{|c|c|c|c|c|}
\hline & & $\mathrm{x}$ & 1 & \\
\hline 1 & C & -0.5537 & 0.4565 & -0.7591 \\
\hline 2 & C & -1.8744 & & \\
\hline 3 & $\Omega$ & 1720 & 8 & \\
\hline 4 & c & 0.7762 & -2 . & . \\
\hline b & c & 1.3193 & 77 & 0 \\
\hline & c & 0 & 09 & 6 \\
\hline & $\mathrm{N}$ & 0 & 88 & 6 \\
\hline & $c$ & 2.3154 & -0 . & 60 \\
\hline & c & 3.6239 & -1 & 1. \\
\hline 0 & c & 4.3116 & -1 & -0 . \\
\hline & o & 4.2015 & -1 & 122 \\
\hline & $\mathrm{H}$ & -2.6713 & 76 & -0.9111 \\
\hline & $\mathrm{H}$ & -2.1542 & 331 & 982 \\
\hline 14 & $\mathrm{H}$ & -1.8234 & -1.0693 & 0.1664 \\
\hline [3] & $\mathrm{H}$ & 0.1459 & -2 . & -0.8895 \\
\hline$[6$ & $\mathrm{F}$ & 0.1673 & -2 . & 0.8894 \\
\hline L & $\mathrm{H}$ & 1.5307 & -2.8206 & -0.0466 \\
\hline L & $\mathrm{H}$ & 1.5628 & 214 & -0.8706 \\
\hline$[5$ & $\mathrm{H}$ & 2.2651 & 1. & $89^{\circ}$ \\
\hline 20 & $\mathrm{H}$ & 1.1 & 3 . & 0 . \\
\hline 21 & $\mathrm{H}$ & 0.3 & 2.6 & 52 \\
\hline 22 & $\mathrm{H}$ & -0. & 320 & 781 \\
\hline 23 & $\mathrm{H}$ & -0.3047 & -0.1242 & -1.6579 \\
\hline 24 & $\mathrm{H}$ & -0.6934 & 1.4823 & -1.1053 \\
\hline 25 & $\mathrm{H}$ & 2.1250 & -0 . & -0.8275 \\
\hline 26 & $\mathrm{H}$ & 54 & -0 . & -0.7145 \\
\hline 27 & 1. & 3.8102 & -2.1351 & -0.5888 \\
\hline 28 & $\mathrm{H}$ & 5.3391 & -1.6098 & R \\
\hline
\end{tabular}

\section{Structure Path1dccF}

$\begin{array}{lrrrr} & \text { Atom } & x & y & z \\ 1 & \mathrm{C} & -0.4342 & 1.3038 & 0.8765 \\ 2 & \mathrm{C} & -1.7264 & 0.9065 & 1.5850 \\ 3 & \mathrm{C} & -0.8347 & -0.3333 & -1.0944 \\ 4 & \mathrm{C} & -2.1916 & 0.2461 & -1.4756 \\ 5 & \mathrm{C} & 1.1828 & 1.1532 & -0.9532 \\ 6 & \mathrm{C} & 2.3224 & 1.6646 & -0.0780 \\ 7 & \mathrm{~N} & 0.1085 & 0.4291 & -0.2125 \\ 8 & \mathrm{O} & -0.8826 & -1.4930 & -0.2812 \\ 9 & \mathrm{C} & 0.4079 & -1.2370 & 0.4513 \\ 10 & \mathrm{C} & 1.5597 & -1.8675 & -0.3250 \\ 11 & \mathrm{O} & 0.3810 & -1.3185 & 1.6775 \\ 12 & \mathrm{H} & -1.8725 & 1.6283 & 2.3956 \\ 13 & \mathrm{H} & -1.6494 & -0.0857 & 2.0229 \\ 14 & \mathrm{H} & -2.6065 & 0.9575 & 0.9478 \\ 15 & \mathrm{H} & -2.1944 & 1.3363 & -1.4017 \\ 16 & \mathrm{H} & -2.9804 & -0.1506 & -0.8366 \\ 17 & \mathrm{H} & -2.4245 & -0.0146 & -2.5102 \\ 18 & \mathrm{H} & 0.7150 & 1.9847 & -1.4936 \\ 19 & \mathrm{H} & 1.5757 & 0.4637 & -1.6992 \\ 20 & \mathrm{H} & 3.1208 & 2.0275 & -0.7310\end{array}$


Table S18. continuation.

$\begin{array}{rrrrr}21 & \mathrm{H} & 2.7364 & 0.8702 & 0.5472 \\ 22 & \mathrm{H} & 2.0218 & 2.4927 & 0.5654 \\ 23 & \mathrm{H} & -0.5340 & 2.3110 & 0.4602 \\ 24 & \mathrm{H} & 0.3608 & 1.3310 & 1.6233 \\ 25 & \mathrm{H} & 2.5158 & -1.4523 & 0.0002 \\ 26 & \mathrm{H} & 1.4723 & -1.7737 & -1.4083 \\ 27 & \mathrm{H} & 1.5477 & -2.9315 & -0.0728 \\ 28 & \mathrm{H} & -0.2669 & -0.5254 & -2.0123\end{array}$

\section{Structure Path1h}

$\begin{array}{lrrrr} & \text { Atom } & x & y & z \\ 1 & \mathrm{C} & -4.3365 & 2.1219 & 3.1041 \\ 2 & \mathrm{C} & -3.2452 & 3.1703 & 3.0107 \\ 3 & \mathrm{~N} & -4.7128 & 1.3892 & 1.8797 \\ 4 & \mathrm{C} & -5.9245 & 0.7231 & 1.9424 \\ 5 & \mathrm{C} & -6.4073 & -0.0626 & 0.9607 \\ 6 & \mathrm{C} & -5.6386 & -0.2431 & -0.2514 \\ 7 & \mathrm{~N} & -4.4409 & 0.4759 & -0.2540 \\ 8 & \mathrm{C} & -3.9185 & 1.3054 & 0.7270 \\ 9 & \mathrm{O} & -2.8745 & 1.9018 & 0.5562 \\ 10 & \mathrm{O} & -5.9522 & -0.9316 & -1.2154 \\ 11 & \mathrm{H} & -3.2121 & 3.6771 & 3.9784 \\ 12 & \mathrm{H} & -3.5015 & 3.9066 & 2.2478 \\ 13 & \mathrm{H} & -2.2715 & 2.7503 & 2.7816 \\ 14 & \mathrm{H} & -3.8707 & 0.4056 & -1.0906 \\ 15 & \mathrm{H} & -7.3631 & -0.5561 & 1.0585 \\ 16 & \mathrm{H} & -6.4749 & 0.8771 & 2.8615 \\ 17 & \mathrm{O} & -4.1001 & 1.1840 & 4.1942 \\ 18 & \mathrm{C} & -3.0759 & 0.2915 & 4.1484 \\ 19 & \mathrm{C} & -3.0092 & -0.4987 & 5.4239 \\ 20 & \mathrm{O} & -2.3418 & 0.1649 & 3.1984 \\ 21 & \mathrm{H} & -2.8191 & 0.1739 & 6.2645 \\ 22 & \mathrm{H} & -2.2131 & -1.2380 & 5.3565 \\ 23 & \mathrm{H} & -3.9648 & -0.9958 & 5.6077 \\ 24 & \mathrm{H} & -5.2523 & 2.6056 & 3.4384\end{array}$

\section{Structure Path2prodN1}

$\begin{array}{lrrrr} & \text { Atom } & x & y & z \\ 1 & \mathrm{C} & -0.7415 & -0.4966 & 1.5071 \\ 2 & \mathrm{O} & 0.0953 & -0.2887 & 2.3673 \\ 3 & \mathrm{~N} & -0.7100 & 0.1202 & 0.2507 \\ 4 & \mathrm{~N} & -1.8059 & -1.3531 & 1.6870 \\ 5 & \mathrm{C} & -2.8130 & -1.6861 & 0.7691 \\ 6 & 0 & -3.6914 & -2.4792 & 1.0821 \\ 7 & \mathrm{C} & -2.6816 & -1.0132 & -0.5064 \\ 8 & \mathrm{C} & -1.6578 & -0.1615 & -0.7126 \\ 9 & \mathrm{C} & 0.3870 & 1.0069 & -0.0489 \\ 10 & \mathrm{C} & 2.6151 & 0.8483 & -0.8651 \\ 11 & 0 & 1.4567 & 0.2013 & -0.5953 \\ 12 & 0 & 2.7503 & 2.0390 & -0.6926 \\ 13 & \mathrm{C} & 3.6613 & -0.0959 & -1.3783 \\ 14 & \mathrm{H} & -1.5202 & 0.3593 & -1.6515\end{array}$


Table S18. continuation.

$\begin{array}{rrrrr}15 & \mathrm{H} & -3.4135 & -1.2055 & -1.2770 \\ 16 & \mathrm{H} & 0.7223 & 1.4963 & 0.8610 \\ 17 & \mathrm{H} & 0.0668 & 1.7417 & -0.7850 \\ 18 & \mathrm{H} & 3.2731 & -0.6704 & -2.2224 \\ 19 & \mathrm{H} & 3.9279 & -0.8044 & -0.5890 \\ 20 & \mathrm{H} & 4.5449 & 0.4633 & -1.6805 \\ 21 & \mathrm{H} & -1.8496 & -1.8062 & 2.5943\end{array}$

\section{Structure TEA}

$\begin{array}{lrrrr} & \text { Atom } & \mathrm{x} & \mathrm{y} & \mathrm{z} \\ 1 & \mathrm{C} & 0.1684 & -0.1443 & -0.1785 \\ 2 & \mathrm{C} & 1.3263 & -0.4703 & -1.1228 \\ 3 & \mathrm{H} & 2.1683 & -0.8769 & -0.5531 \\ 4 & \mathrm{H} & 1.0231 & -1.2157 & -1.8640 \\ 5 & \mathrm{H} & 1.6895 & 0.4119 & -1.6566 \\ 6 & \mathrm{~N} & -1.0318 & 0.3451 & -0.8732 \\ 7 & \mathrm{C} & -2.2714 & 0.0689 & -0.1281 \\ 8 & \mathrm{C} & -2.4616 & 0.7907 & 1.2160 \\ 9 & \mathrm{H} & -3.4077 & 0.4754 & 1.6680 \\ 10 & \mathrm{H} & -1.6622 & 0.5537 & 1.9240 \\ 11 & \mathrm{H} & -2.4999 & 1.8774 & 1.0993 \\ 12 & \mathrm{H} & -3.1171 & 0.3022 & -0.7804 \\ 13 & \mathrm{H} & -2.3061 & -1.0121 & 0.0410 \\ 14 & \mathrm{C} & -0.8890 & 1.7409 & -1.3099 \\ 15 & \mathrm{C} & -1.9657 & 2.1953 & -2.2940 \\ 16 & \mathrm{H} & -1.7269 & 3.1974 & -2.6632 \\ 17 & \mathrm{H} & -2.0181 & 1.5197 & -3.1534 \\ 18 & \mathrm{H} & -2.9577 & 2.2438 & -1.8376 \\ 19 & \mathrm{H} & -0.8615 & 2.4331 & -0.4498 \\ 20 & \mathrm{H} & 0.0824 & 1.8295 & -1.8012 \\ 21 & \mathrm{H} & 0.5146 & 0.5711 & 0.5877 \\ 22 & \mathrm{H} & -0.1113 & -1.0578 & 0.3542\end{array}$

\section{Structure TEAHCI}

$\begin{array}{lrrrr} & \text { Atom } & x & y & z \\ 1 & \mathrm{C} & -1.3915 & 0.7392 & 0.1380 \\ 2 & \mathrm{C} & -2.6513 & -0.1043 & 0.2713 \\ 3 & \mathrm{~N} & -0.1912 & -0.0268 & -0.3694 \\ 4 & \mathrm{C} & 0.9534 & 0.9033 & -0.7079 \\ 5 & \mathrm{C} & 1.6002 & 1.5923 & 0.4838 \\ 6 & \mathrm{C} & 0.1863 & -1.1704 & 0.5405 \\ 7 & \mathrm{C} & 1.3867 & -1.9704 & 0.0570 \\ 8 & \mathrm{H} & -3.4856 & 0.5687 & 0.4859 \\ 9 & \mathrm{H} & -2.8778 & -0.6346 & -0.6564 \\ 10 & \mathrm{H} & -2.5926 & -0.8254 & 1.0882 \\ 11 & \mathrm{H} & 2.4081 & 2.2243 & 0.1059 \\ 12 & \mathrm{H} & 0.9043 & 2.2379 & 1.0230 \\ 13 & \mathrm{H} & 2.0399 & 0.8827 & 1.1878 \\ 14 & \mathrm{H} & 1.6802 & 0.3067 & -1.2570 \\ 15 & \mathrm{H} & 0.5379 & 1.6328 & -1.4040 \\ 16 & \mathrm{H} & 1.4979 & -2.8405 & 0.7091 \\ 17 & \mathrm{H} & 1.2413 & -2.3335 & -0.9631\end{array}$


Table S18. continuation.

$\begin{array}{rrrrr}18 & \mathrm{H} & 2.3175 & -1.4025 & 0.1006 \\ 19 & \mathrm{H} & 0.3550 & -0.7514 & 1.5336 \\ 20 & \mathrm{H} & -0.6894 & -1.8150 & 0.5883 \\ 21 & \mathrm{H} & -1.1142 & 1.1855 & 1.0929 \\ 22 & \mathrm{H} & -1.5508 & 1.5407 & -0.5844 \\ 23 & \mathrm{Cl} & -1.0062 & -1.0752 & -3.2047 \\ 24 & \mathrm{H} & -0.4761 & -0.4440 & -1.2955\end{array}$

\section{Structure $\mathrm{UCH}_{2} \mathrm{Cl}_{2}$}

$\begin{array}{lrrrr} & \text { Atom } & \text { x } & y & z \\ 1 & \mathrm{C} & -0.3732 & 1.3776 & -1.0163 \\ 2 & \mathrm{O} & 0.8322 & 1.2194 & -1.1240 \\ 3 & \mathrm{~N} & -1.0253 & 2.5229 & -1.4332 \\ 4 & \mathrm{H} & -0.4467 & 3.2458 & -1.8431 \\ 5 & \mathrm{H} & -2.7472 & 3.6557 & -1.6860 \\ 6 & \mathrm{~N} & -1.2185 & 0.4337 & -0.4677 \\ 7 & \mathrm{H} & -4.2473 & 1.9189 & -0.6848 \\ 8 & \mathrm{H} & -0.7768 & -0.4224 & -0.1493 \\ 9 & \mathrm{C} & -2.6104 & 0.5228 & -0.3014 \\ 10 & \mathrm{O} & -3.2251 & -0.4076 & 0.2069 \\ 11 & \mathrm{C} & -3.1819 & 1.7673 & -0.7748 \\ 12 & \mathrm{C} & -2.3778 & 2.7082 & -1.3158\end{array}$

\section{Details of reactions considered in the text.}

All the molecular structures were optimized with the Berny Optimization Algorithm (default) implemented in the Gaussian 16 suite of programs. All the harmonic frequencies were positive except the case of the transition state structure for which one negative frequency was obtained.

The Gibbs free energies were labeled with $\mathrm{L}-$ and $\mathrm{R}$-codes for convenience. $\mathrm{L}$ corresponds to the lefthand side and $\mathrm{R}$ to the right-hand side of the reaction considered.

The codes $\mathrm{L} 1, \mathrm{~L} 2, \ldots \mathrm{R} 1, \mathrm{R} 2, \ldots$ are explained in the Table S17.

\section{Eq.1}

$\mathrm{U}+2 \mathrm{Ac}_{2} \mathrm{O}+\mathrm{TEA}+\mathrm{CH}_{2} \mathrm{Cl}_{2}+2 \mathrm{DMAP} \rightarrow 3 \mathrm{c}+\mathrm{N}$-Ac-diethylamine $+\mathrm{CH}_{3} \mathrm{Cl}+\left[N^{1}-\mathrm{Ac} \mathrm{DMAP}\right]^{(+)} \mathrm{Cl}^{(-)}$ $+[\mathrm{DMAPH}]^{(+)} \mathrm{CH}_{3} \mathrm{COO}^{(-)}$

$\mathrm{U}+2 \mathrm{Ac}_{2} \mathrm{O}+\mathrm{TEA}+\mathrm{CH}_{2} \mathrm{Cl}_{2}+2 \mathrm{DMAP} \rightarrow$
(L1)
(L2)
(L3)
(L4)
(L5)

product 3c $+N$-acyl-diamine $+\mathrm{CH}_{3} \mathrm{Cl}+\left[N^{1}\right.$-acetylDMAP $] \mathrm{Cl}+[$ DMAP*H $] \mathrm{CH} 3 \mathrm{COO}$
(R1)
(R2)
(R3)
(R4)
(R5) 


\section{Uphill Transition state (TS)}

$N$-1-acetyloxyethyl diethylamine

$$
\rightarrow \quad \mathrm{TS}
$$

\section{Downhill Transition state (TS)}

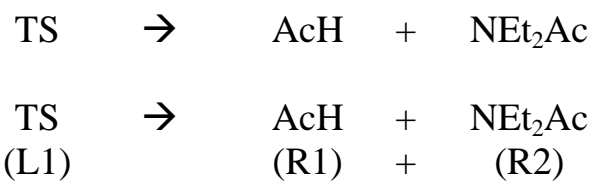

\section{Eq.2}

$\mathrm{U}+\mathrm{TEA}+\mathrm{CH}_{2} \mathrm{Cl}_{2}+\mathrm{Ac}_{2} \mathrm{O} \rightarrow \mathbf{3 b}+[\mathrm{TEA} \times \mathrm{HCl}]+\mathrm{AcCl}$

$$
\mathrm{U}+\mathrm{TEA}+\mathrm{CH}_{2} \mathrm{Cl}_{2}+\mathrm{Ac}_{2} \mathrm{O} \rightarrow \text { product } 3 \mathbf{b}+[\mathrm{TEA} * \mathrm{HCl}]+\mathrm{AcCl}
$$
(L1) (L2)
(L3)
(L4)
(R1)
(R2)
(R3)

The reaction output is calculated with the Gibbs free energy values (Table S17) as follows (1 Hartree $=627.5 \mathrm{kcal} / \mathrm{mol})$ :

\section{Eq.1:}

$\mathrm{L}=\mathrm{L} 1+2 \mathrm{~L} 2+\mathrm{L} 3+\mathrm{L} 4+2 \mathrm{~L} 5$

$\mathrm{R}=\mathrm{R} 1+\mathrm{R} 2+\mathrm{R} 3+\mathrm{R} 4+\mathrm{R} 5$

Output $=\mathrm{R}-\mathrm{L}=-10.07 \mathrm{kcal} / \mathrm{mol}$

\section{Uphill TS:}

$\mathrm{L}=\mathrm{L} 1$

$\mathrm{R}=\mathrm{R} 1$

Output $=\mathrm{R}-\mathrm{L}=26.97 \mathrm{kcal} / \mathrm{mol}$

\section{Downhill TS:}

$\mathrm{L}=\mathrm{L} 1$

$\mathrm{R}=\mathrm{R} 1+\mathrm{R} 2$

Output $=\mathrm{R}-\mathrm{L}=-53.94 \mathrm{kcal} / \mathrm{mol}$

\section{Eq.2:}

$\mathrm{L}=\mathrm{L} 1+\mathrm{L} 2+\mathrm{L} 3+\mathrm{L} 4$

$\mathrm{R}=\mathrm{R} 1+\mathrm{R} 2+\mathrm{R} 3$

Output $=\mathrm{R}-\mathrm{L}=-18.52 \mathrm{kcal} / \mathrm{mol}$ 


\section{References:}

1. CrysAlis CCD and CrysAlis RED; Oxford Diffraction Ltd: Yarnton, 2008.

2. G. M. Sheldrick, Acta Crystallogr. Sect. A, 64 (2008), 112-122.

3. O. V. Dolomanov, L. J. Bourhis, R. J. Gildea, J. A. K. Howard, H. Puschmann, J. Appl. Cryst., 42 (2009), 339-341.

4. C. F. Macrae, P. R. Edgington, P. McCabe, E. Pidcock, G. P. Shields, R. Taylor, M. Towler, J. van de Streek, J. Appl. Cryst., 39 (2006), 453-457. 
1H NMR, $500 \mathrm{MHz}, \mathrm{CDCl} 3$
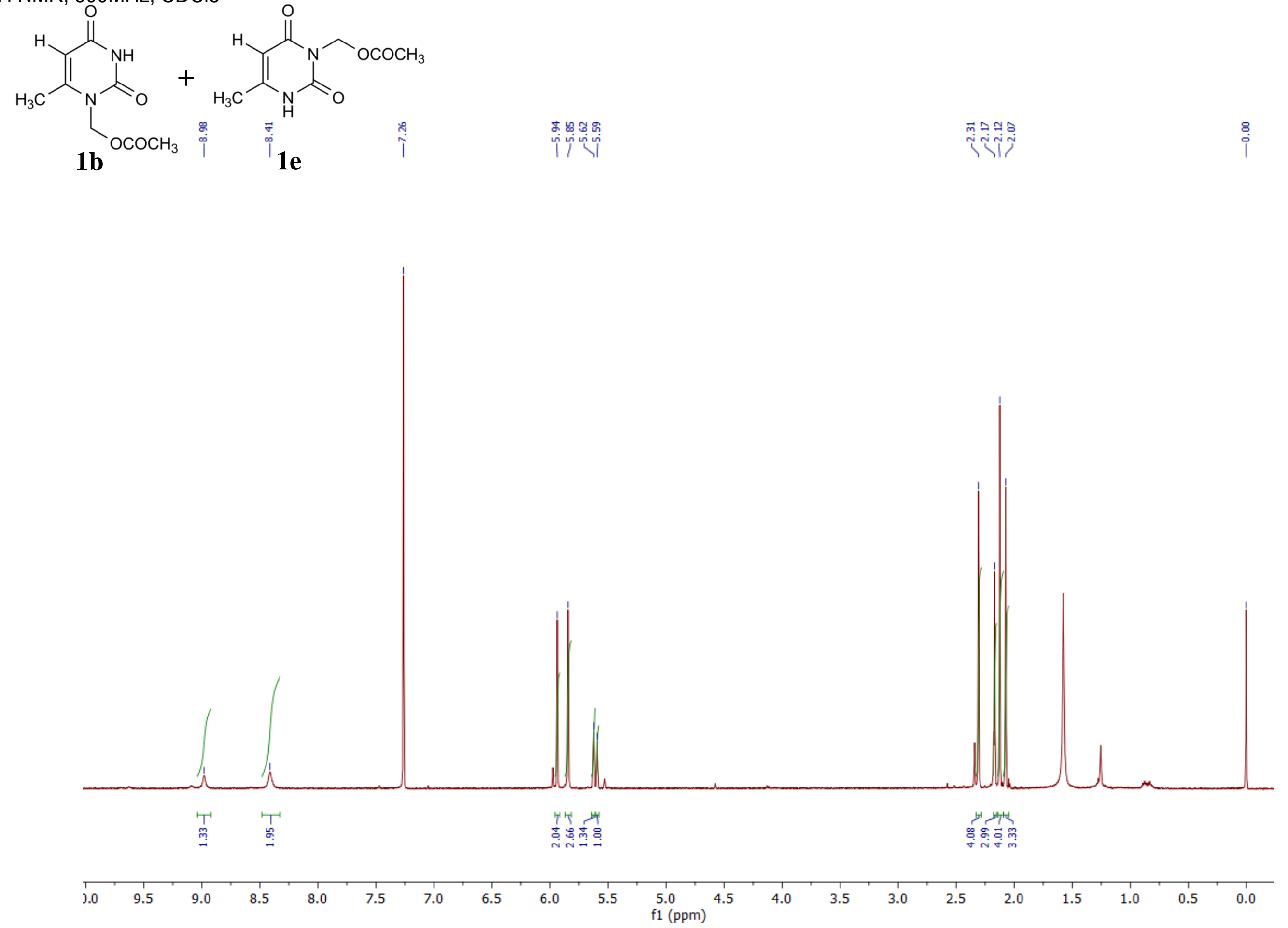


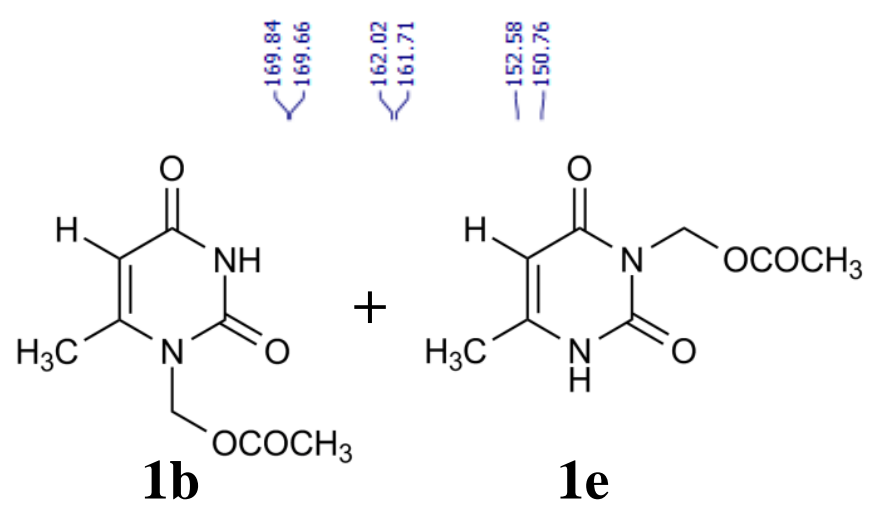



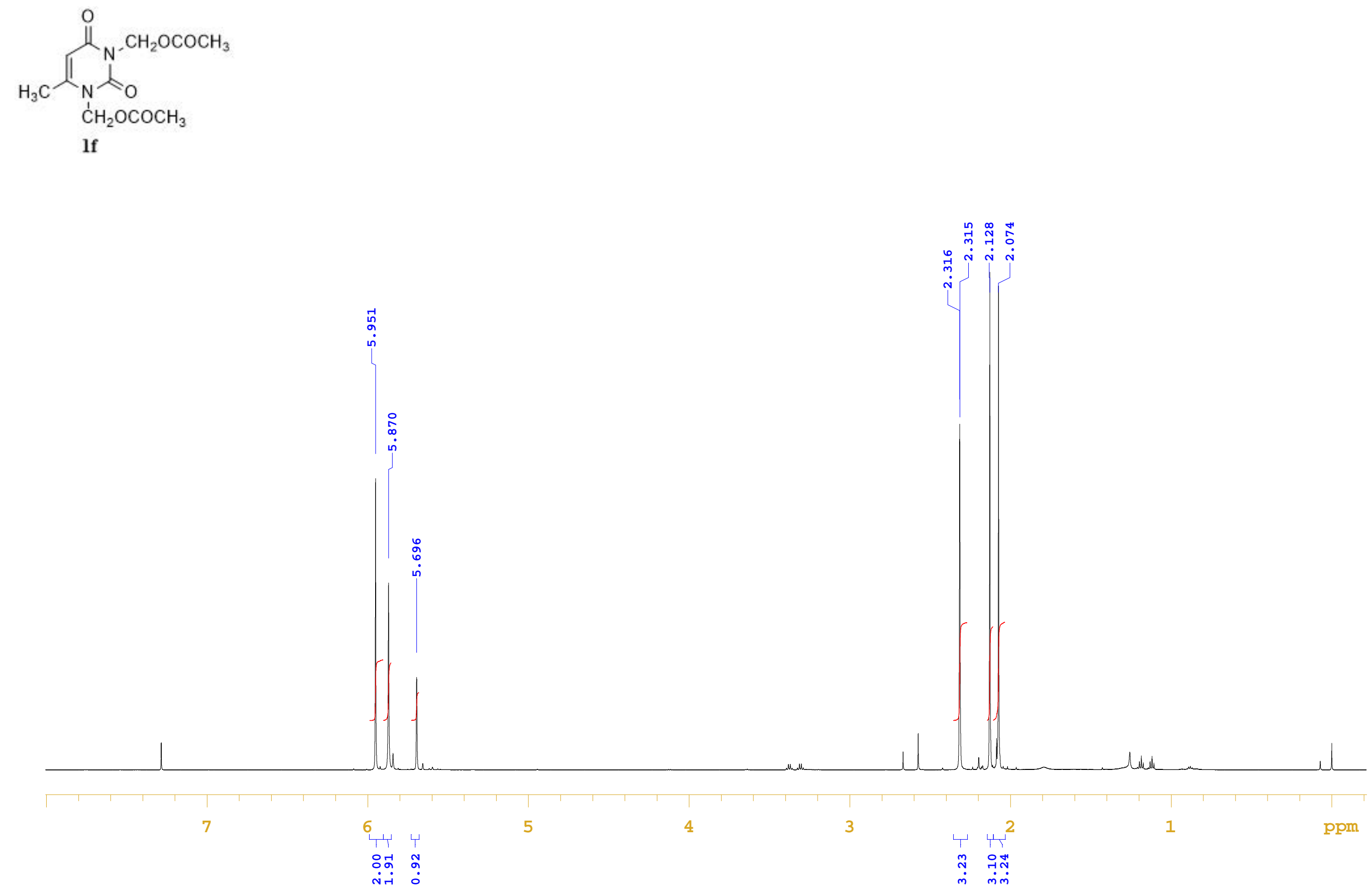

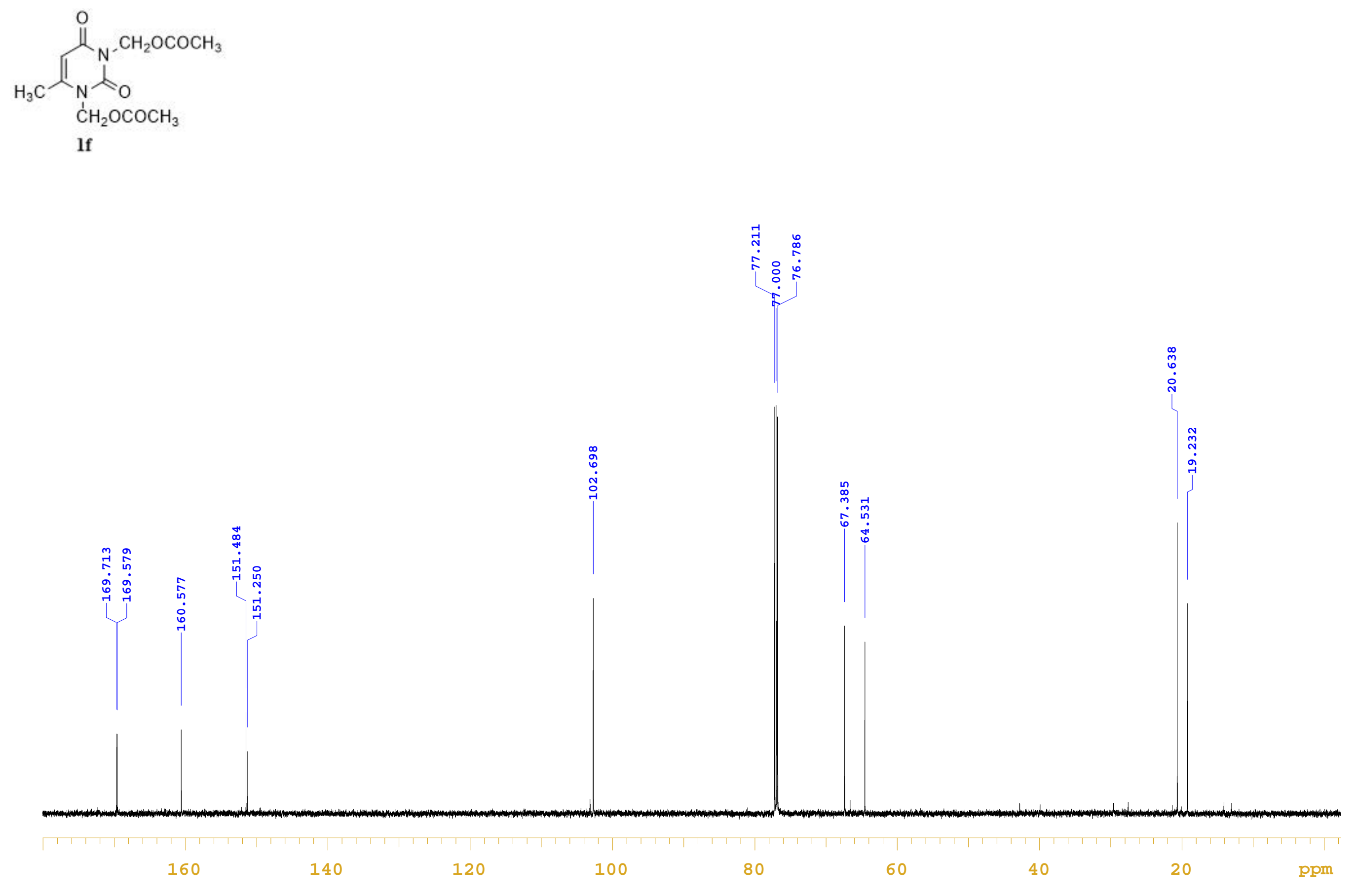

$13 \mathrm{C}\{1 \mathrm{H}\} \mathrm{NMR}, 150 \mathrm{MHz}, \mathrm{CDCl} 3$<smiles>CC(=O)n1cc(C)c(=O)[nH]c1=O</smiles>

2a

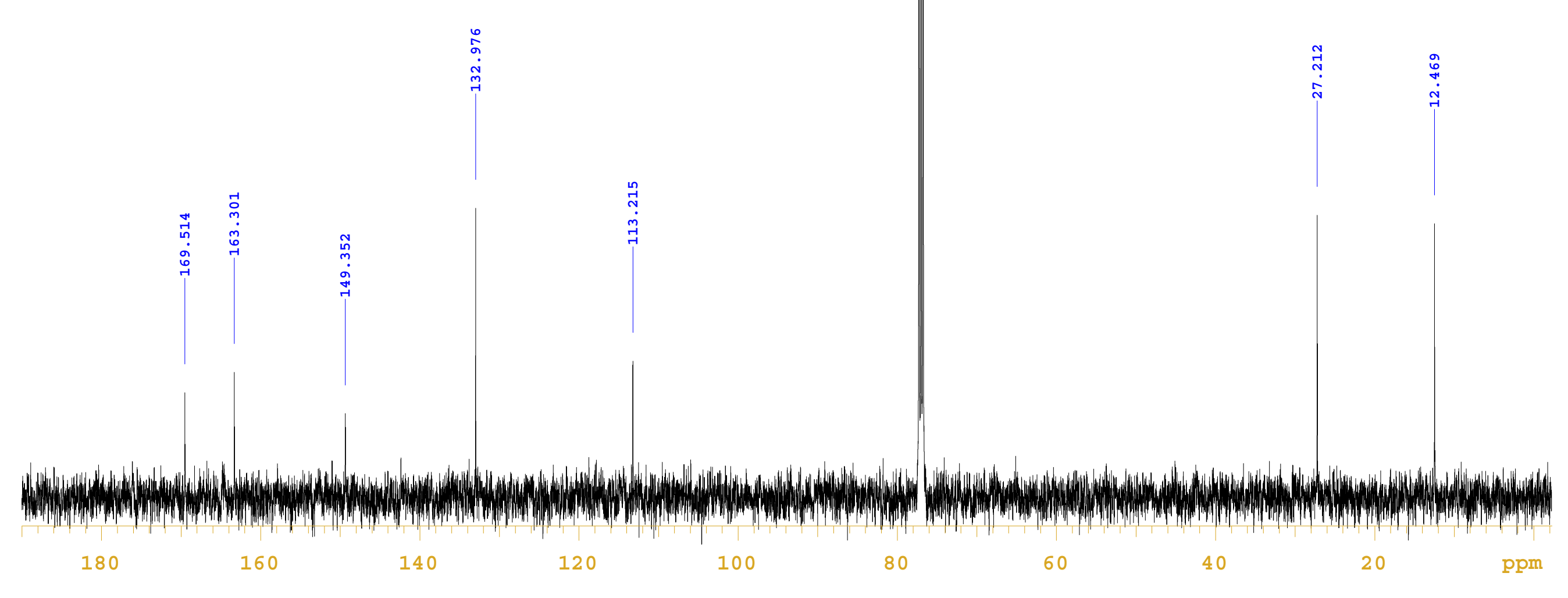


1H NMR, 600MHz, CDCl3
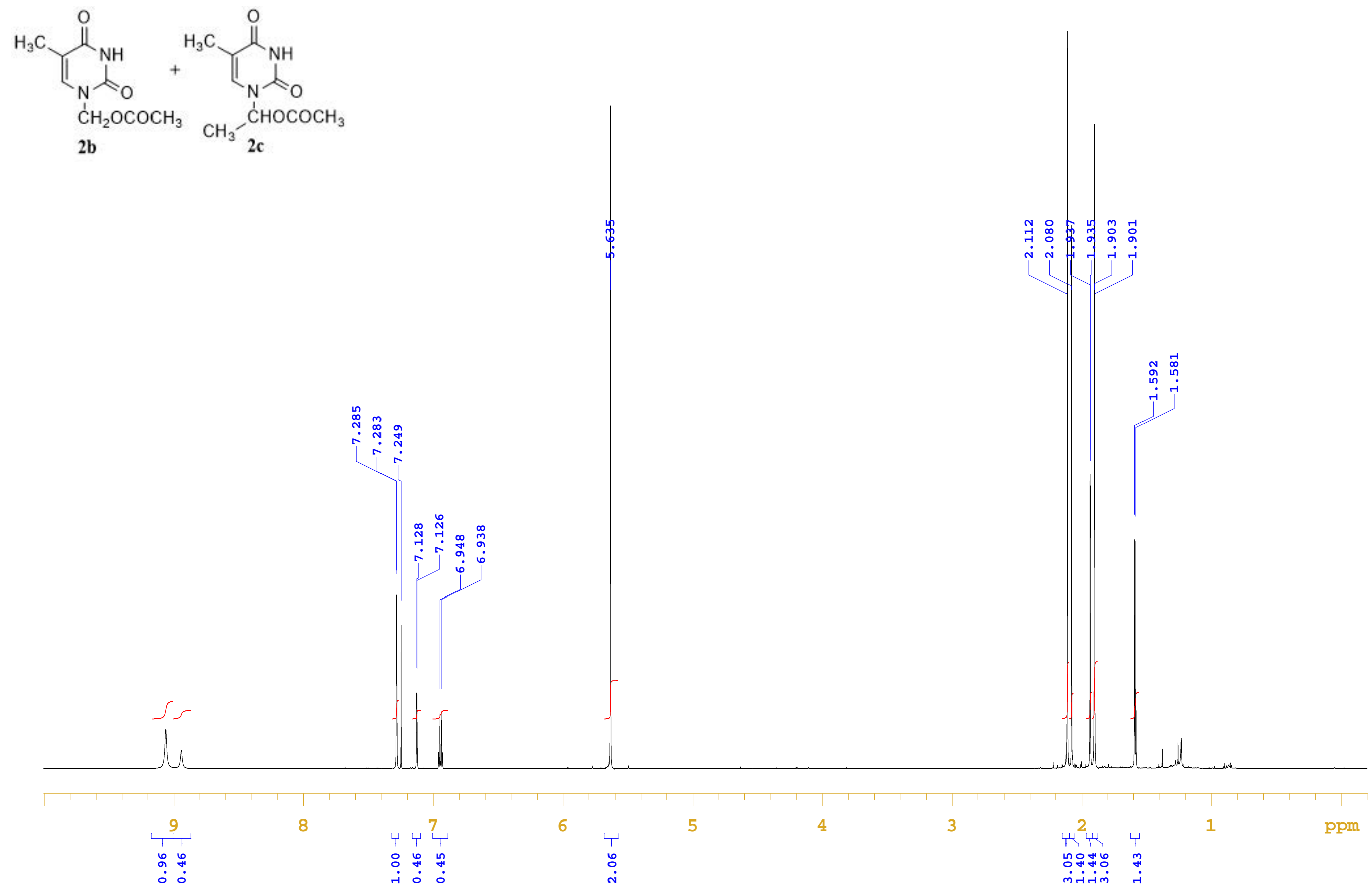
$13 \mathrm{C}\{1 \mathrm{H}\} \mathrm{NMR}, 150 \mathrm{MHz}, \mathrm{CDCl} 3$
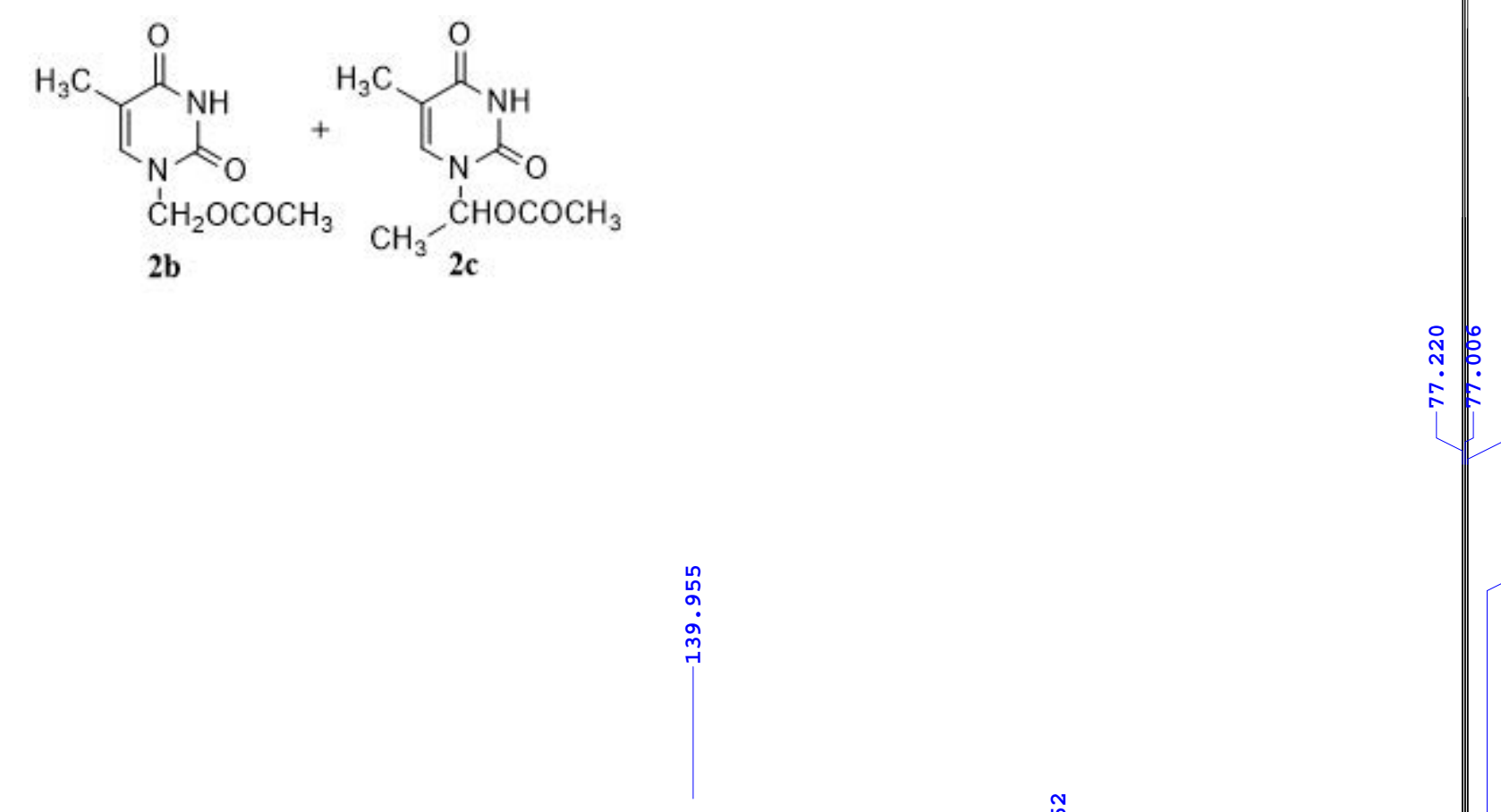

$$
\text { |c }
$$

1 i

เิ

苞

品

ซู 
1H NMR, 600MHz, CDCl
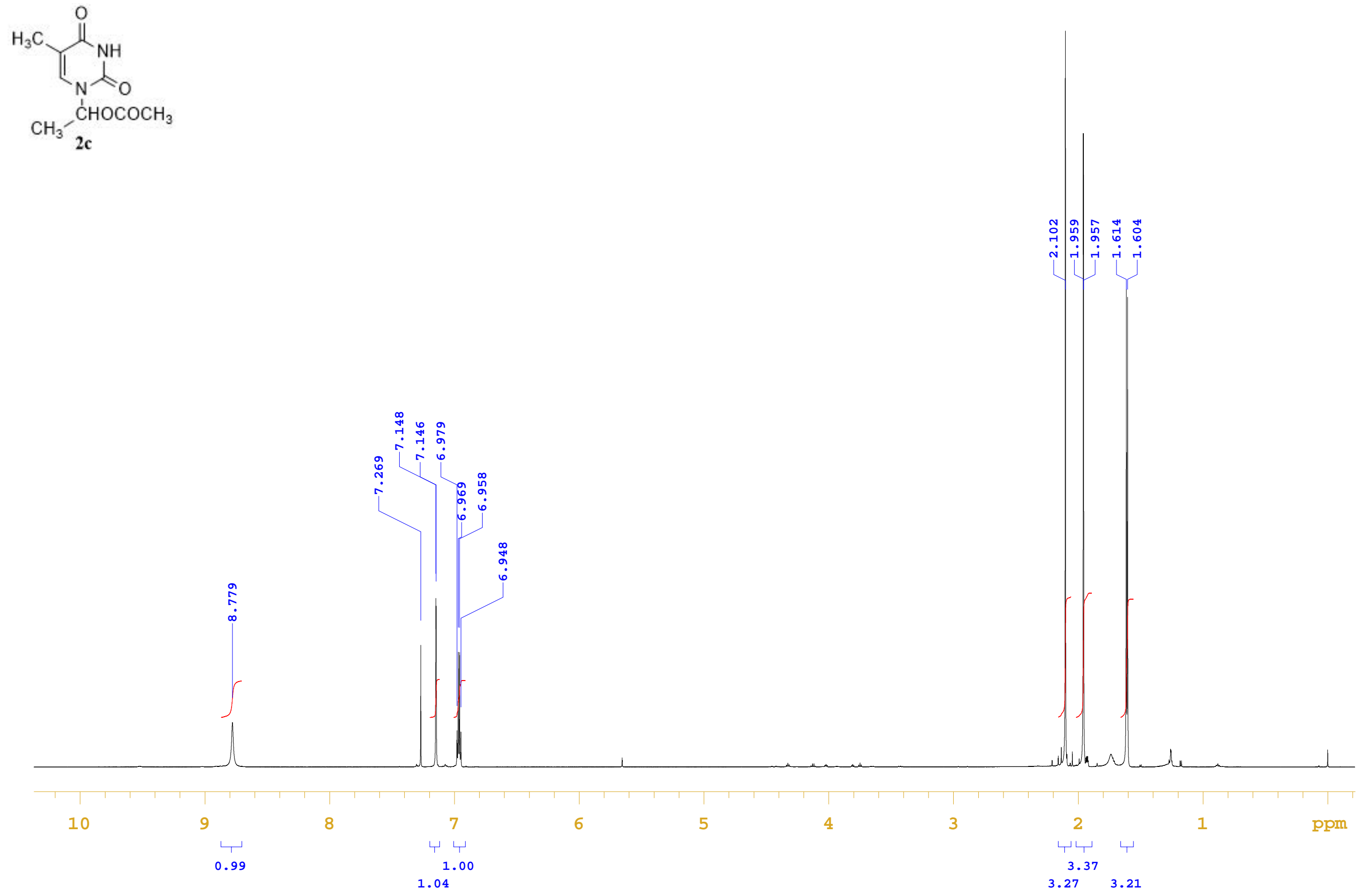
$13 \mathrm{C}\{1 \mathrm{H}\} \mathrm{NMR}, 150 \mathrm{MHz}, \mathrm{CDCl} 3$

$$
\text { 至 }
$$
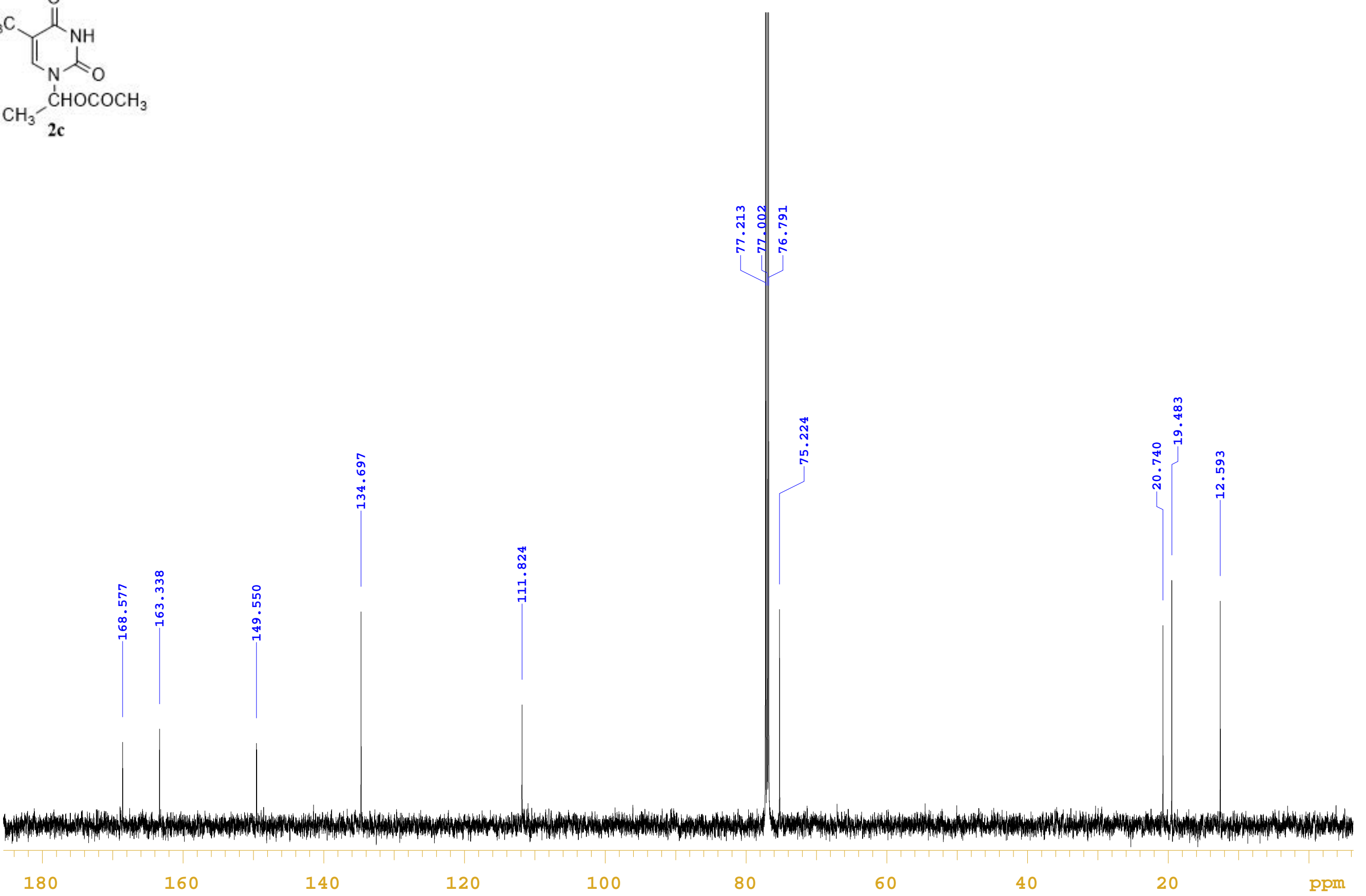


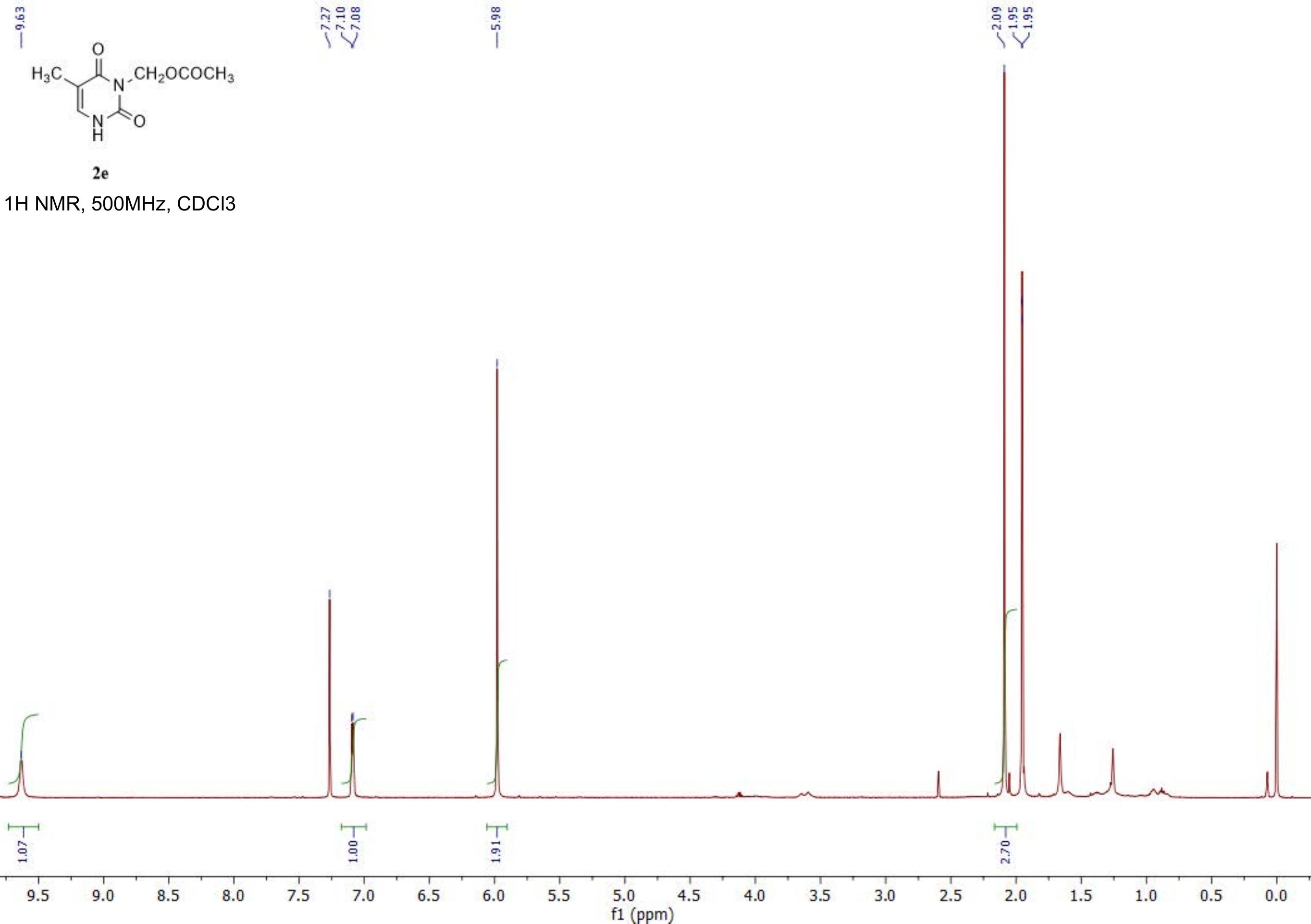




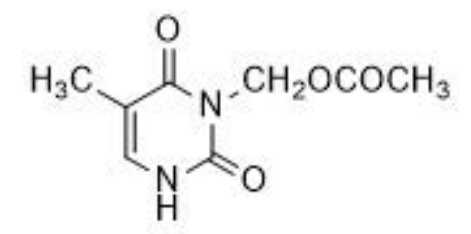

$2 \mathrm{e}$

$13 \mathrm{C}\{1 \mathrm{H}\} \mathrm{NMR}, 125 \mathrm{MHz}, \mathrm{CDCl} 3$ 

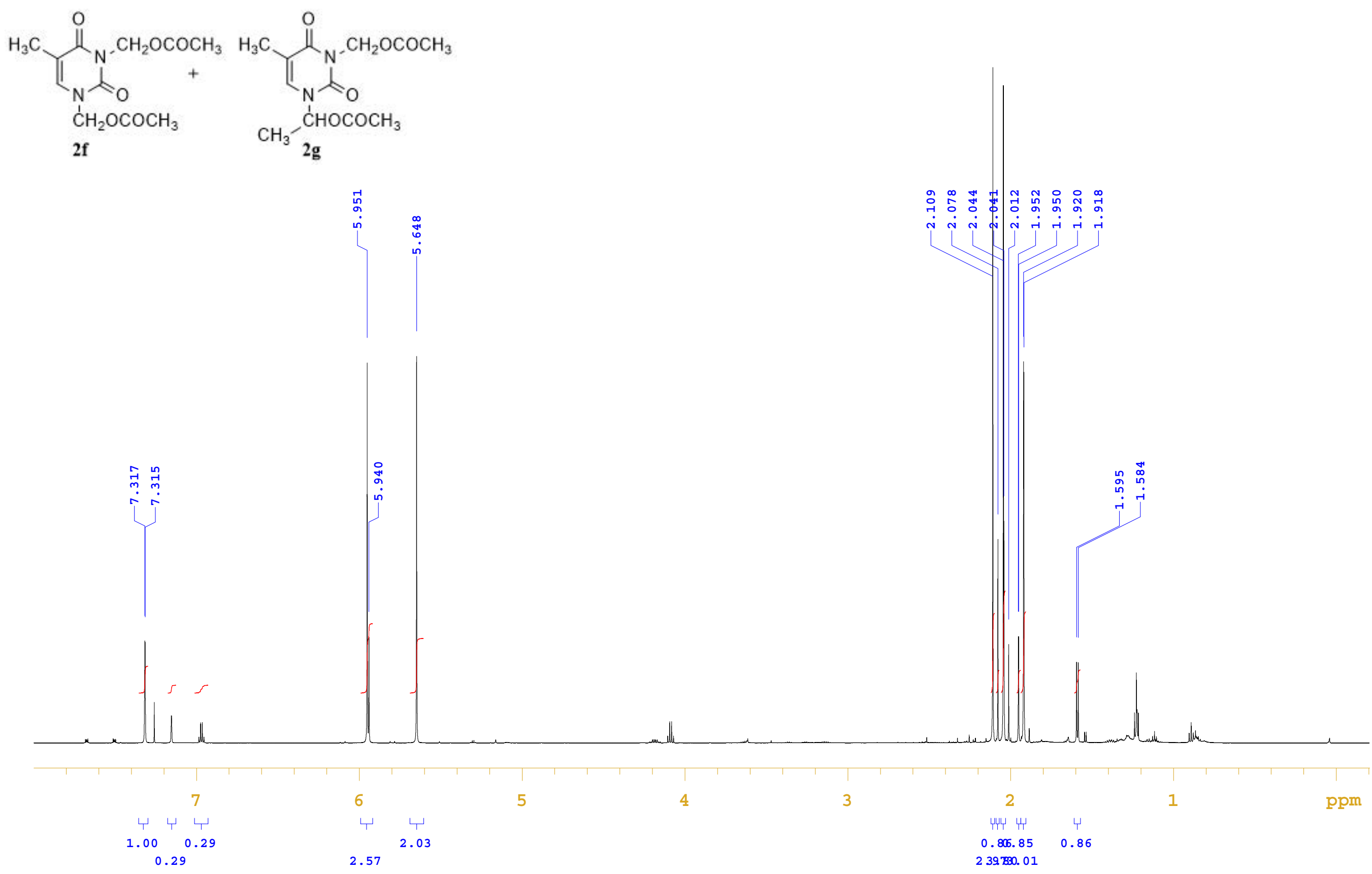

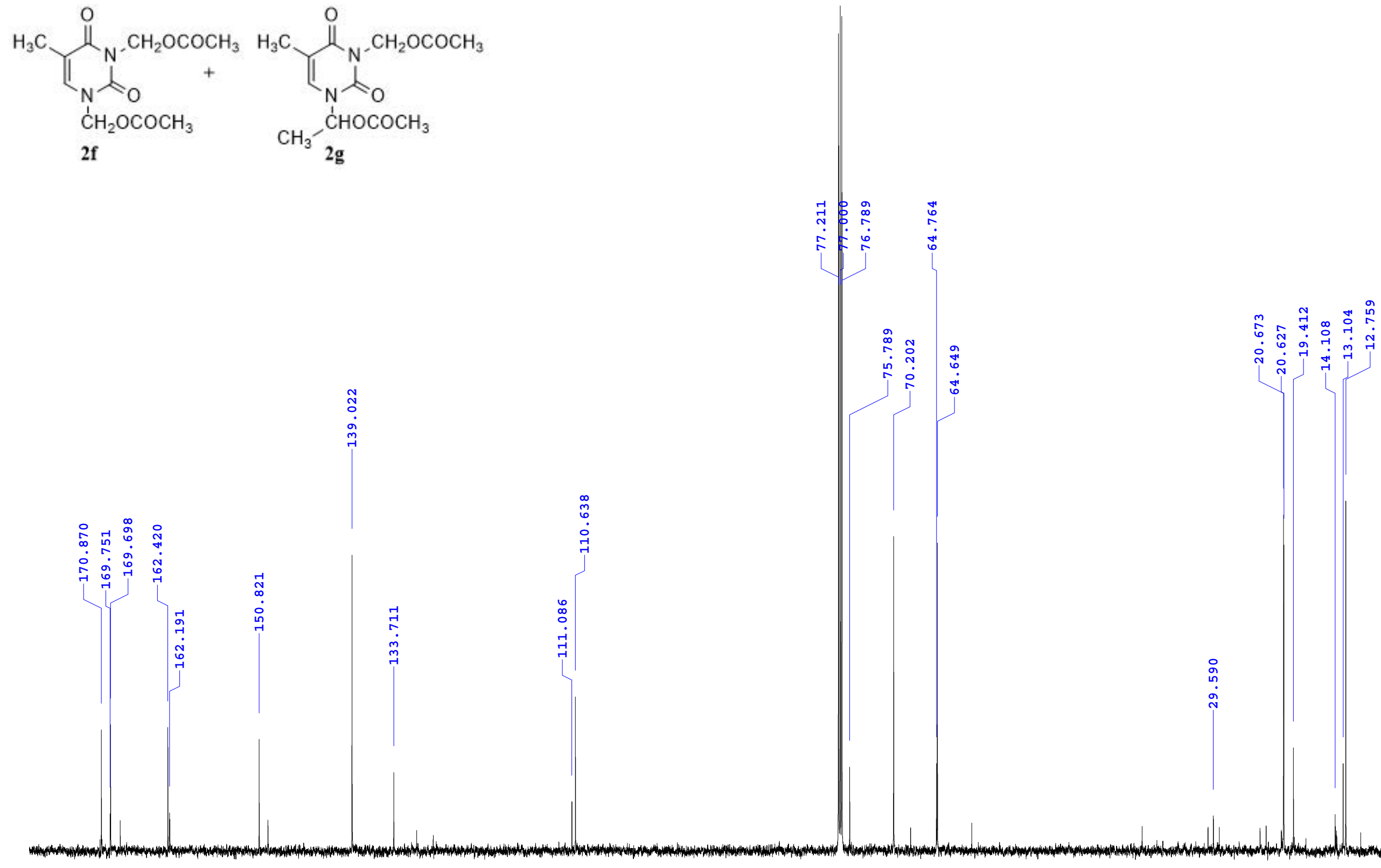

13C $\{1 \mathrm{H}\} \mathrm{NMR}, 150 \mathrm{MHz}, \mathrm{CDCl} 3$

$\mathrm{H}_{3} \mathrm{C}{ }_{\mathrm{O}}^{-\mathrm{CH}_{2} \mathrm{CH}_{2} \mathrm{Cl}}$

$2 \mathbf{i}$

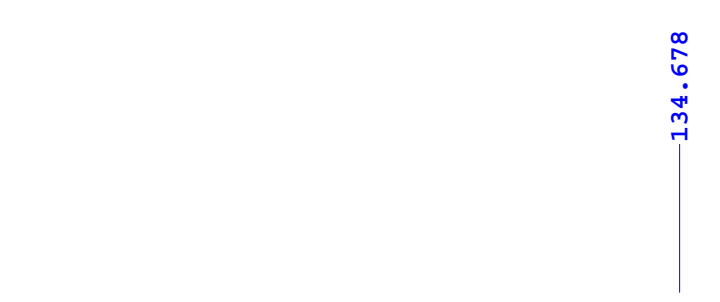

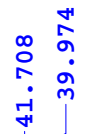

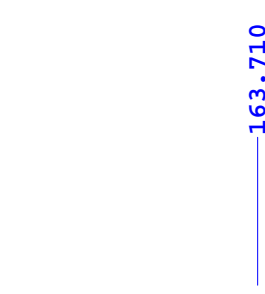

$\stackrel{\leftarrow}{\circ}$

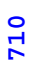

$\stackrel{\leftrightarrow}{\circ}$

กั

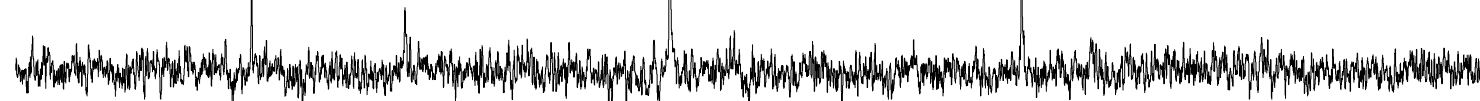

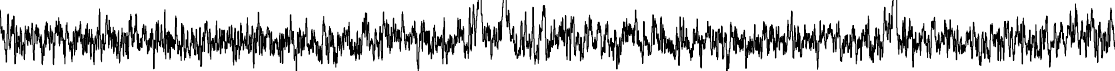

160

140

120

100

80

60

40

20

ppm 


\section{H NMR, 500MHz, CDCl3}

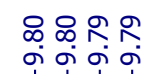

능뉴웜우

氙

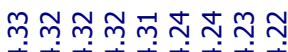

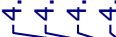

ํํㅇํํㅇํำ

نंi्iन

$\mathrm{H}_{3} \mathrm{C}{ }_{\mathrm{O}}^{\mathrm{C}} \mathrm{CH}_{2} \mathrm{CH}_{2} \mathrm{OCOCH}_{3}$

$2 \mathrm{j}$

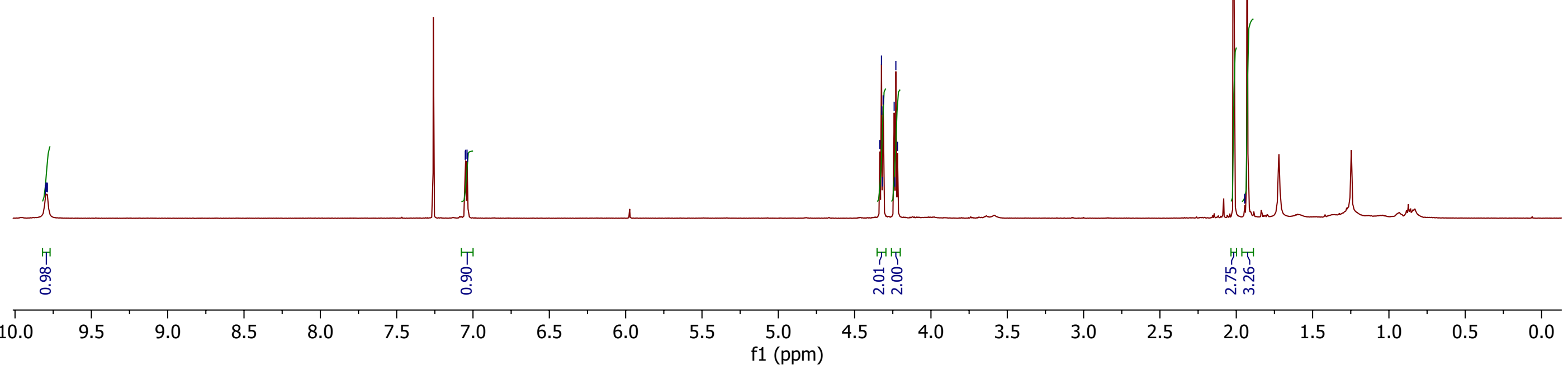


$13 \mathrm{C}\{1 \mathrm{H}\} \mathrm{NMR}, 125 \mathrm{MHz}, \mathrm{CDCl} 3$

\begin{tabular}{|c|c|c|c|c|c|c|}
\hline 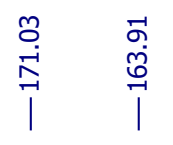 & 离 & 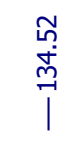 & $\begin{array}{l}0 \\
0 \\
0 \\
0 \\
1\end{array}$ & 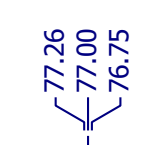 & i & \\
\hline
\end{tabular}<smiles>COCCn1c(=O)[nH]cc(C)c1=O</smiles>

$2 \mathrm{j}$

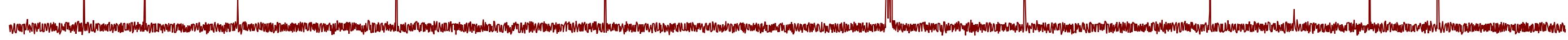



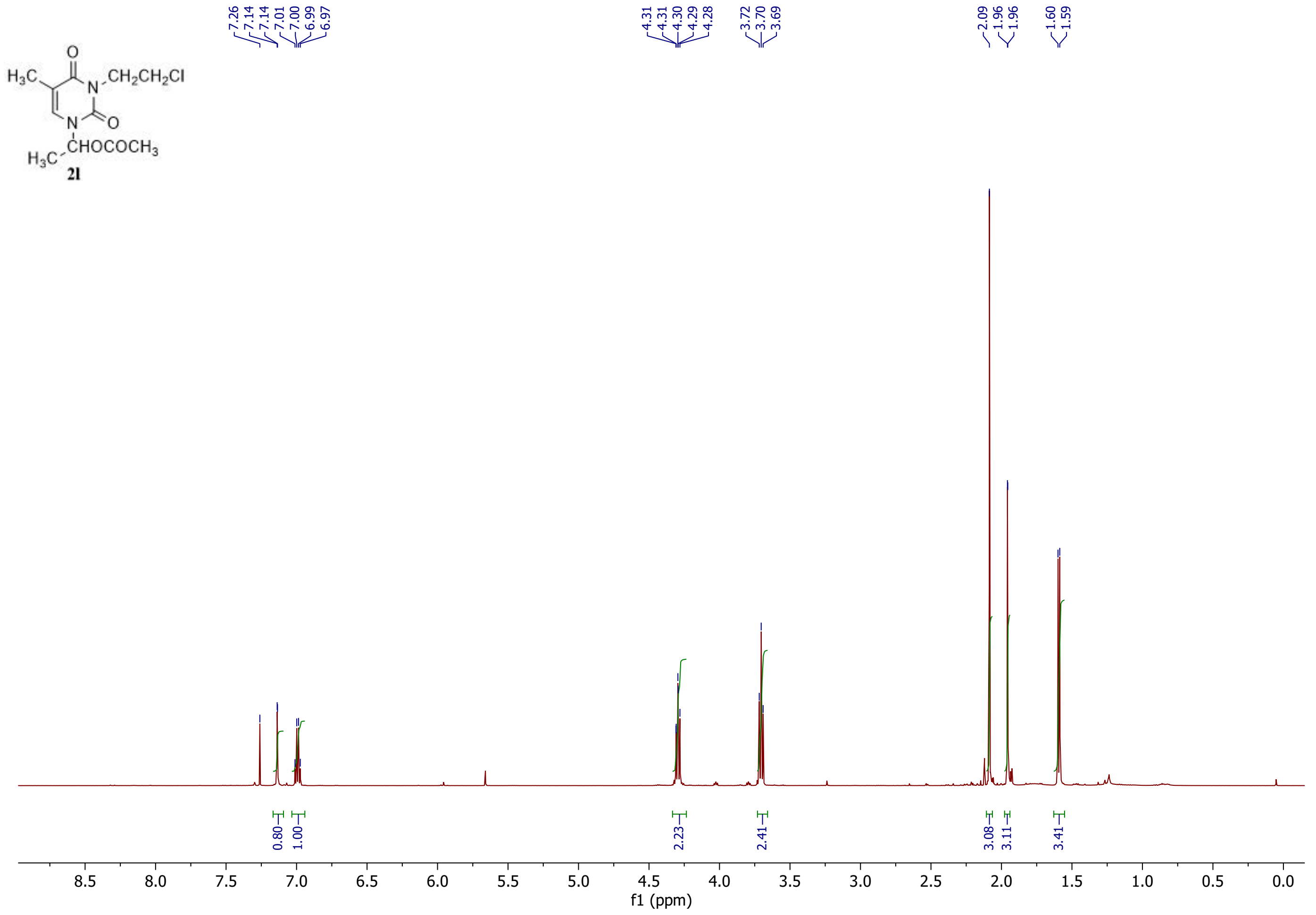
$13 \mathrm{C}\{1 \mathrm{H}\} \mathrm{NMR}, 125 \mathrm{MHz}, \mathrm{CDCl} 3$

$$
\begin{array}{lll}
\infty & m & \\
\infty & \infty & - \\
\infty & 0 & 0 \\
0 & 1 & 1
\end{array}
$$
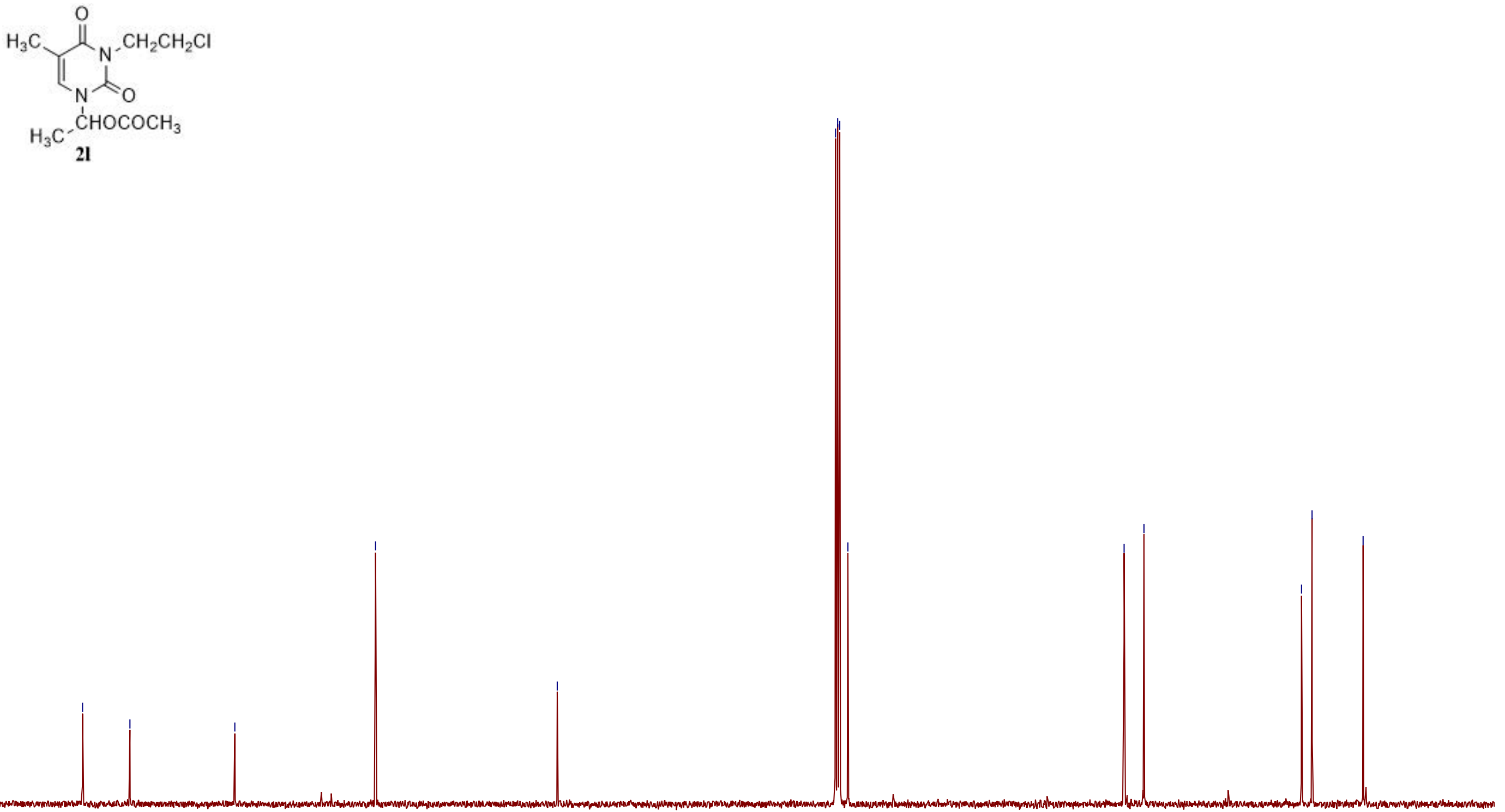
1H NMR, 600MHz, CDCl3
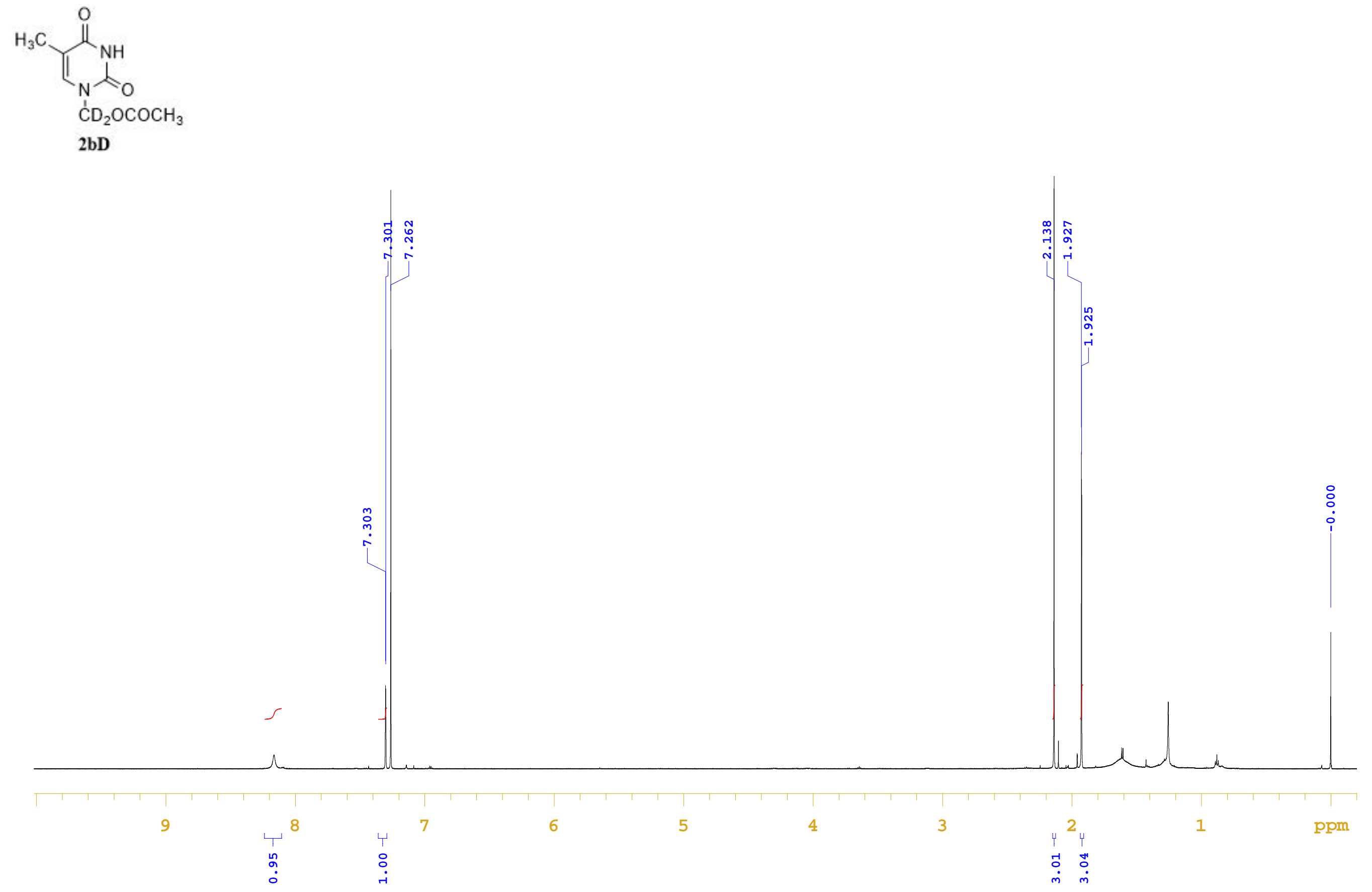
13C $\{1 \mathrm{H}\} \mathrm{NMR}, 150 \mathrm{MHz}, \mathrm{CDCl} 3$
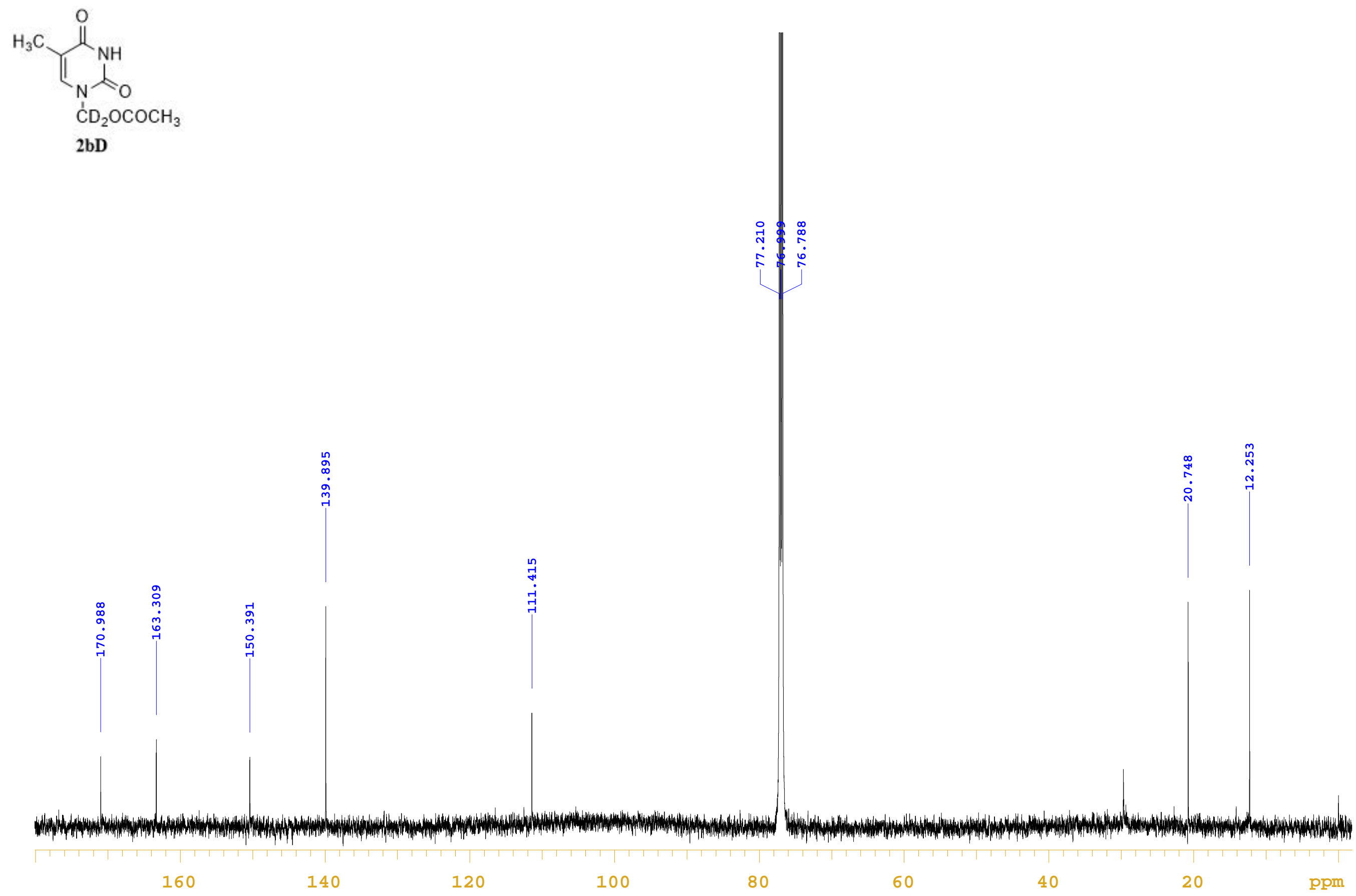
1H NMR, 600MHz, CDCl3

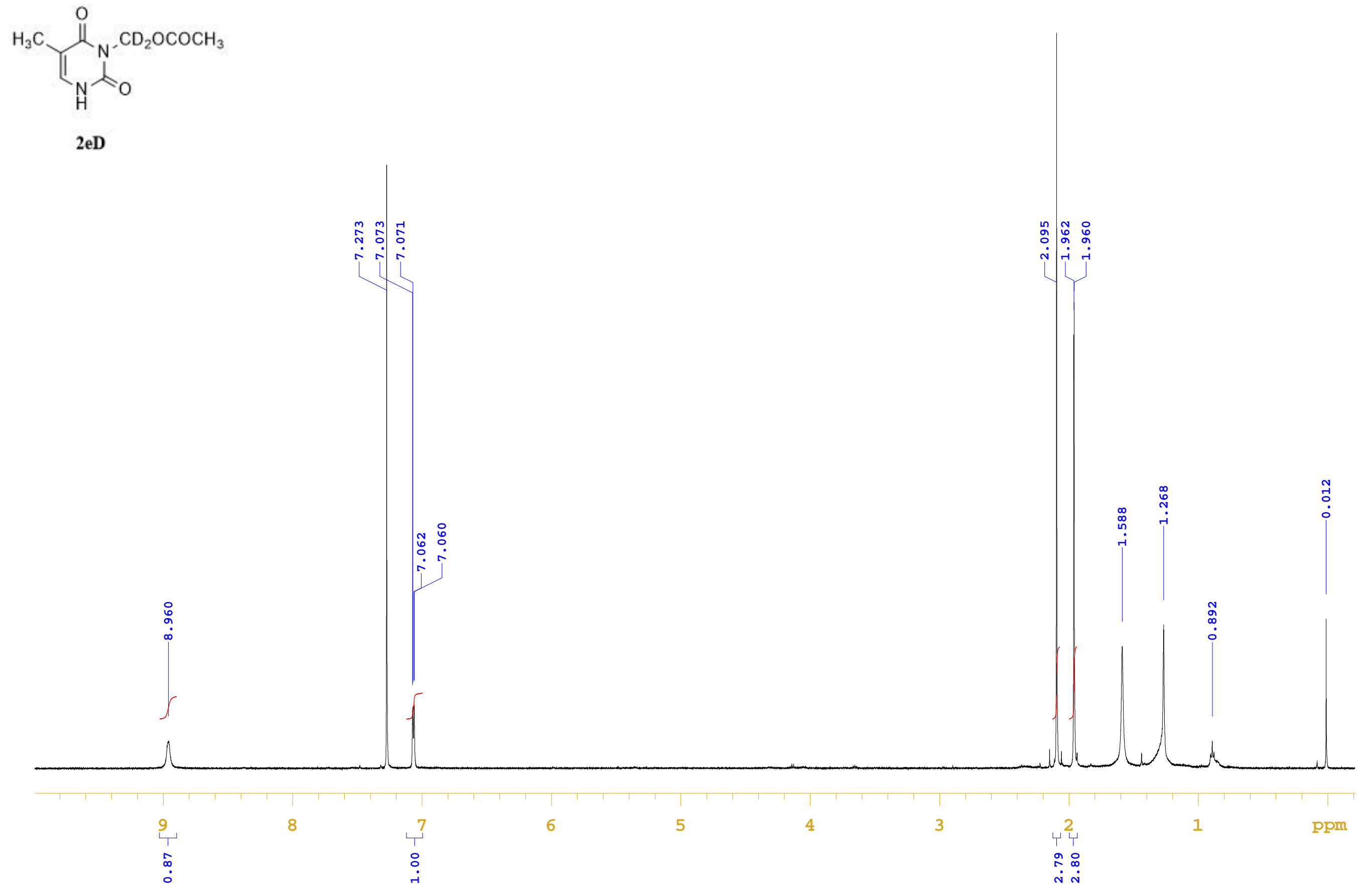


$13 \mathrm{C}\{1 \mathrm{H}\} \mathrm{NMR}, 150 \mathrm{MHz}, \mathrm{CDCl} 3$

${ }_{3} \mathrm{C} \overbrace{\mathrm{N}} \mathrm{CD}_{2} \mathrm{OCOCH}_{3}$

2eD
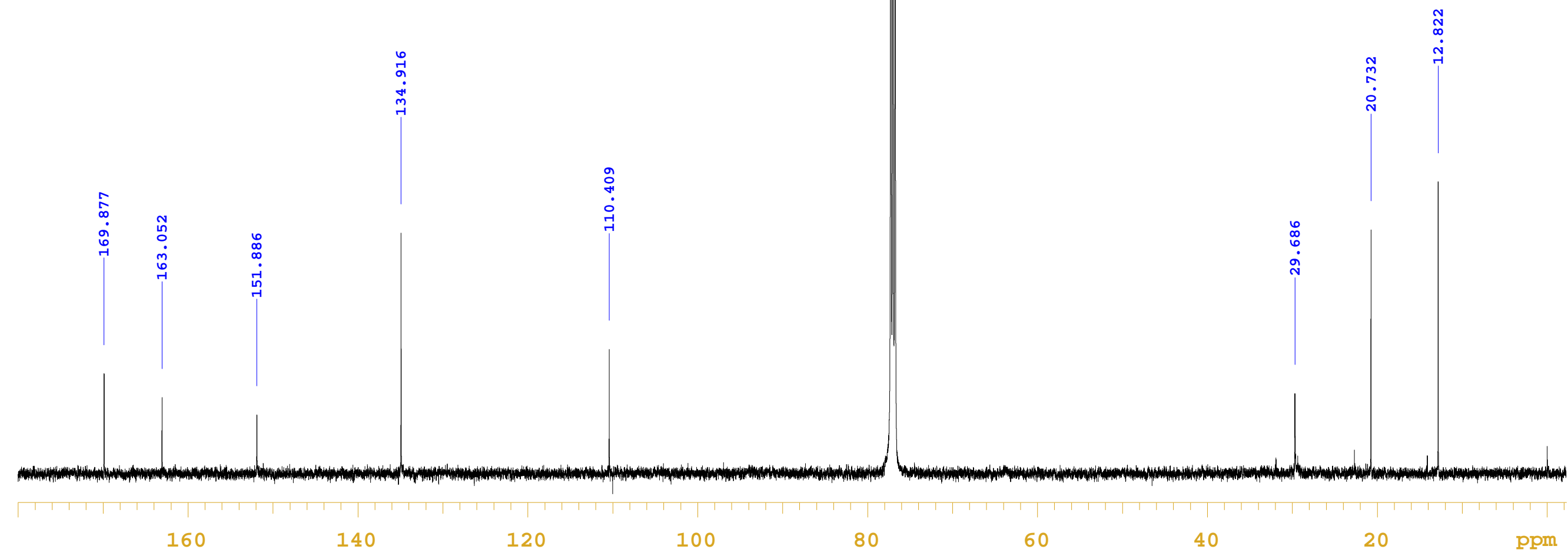
N

2fD

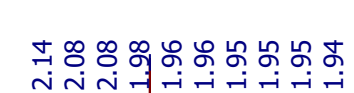
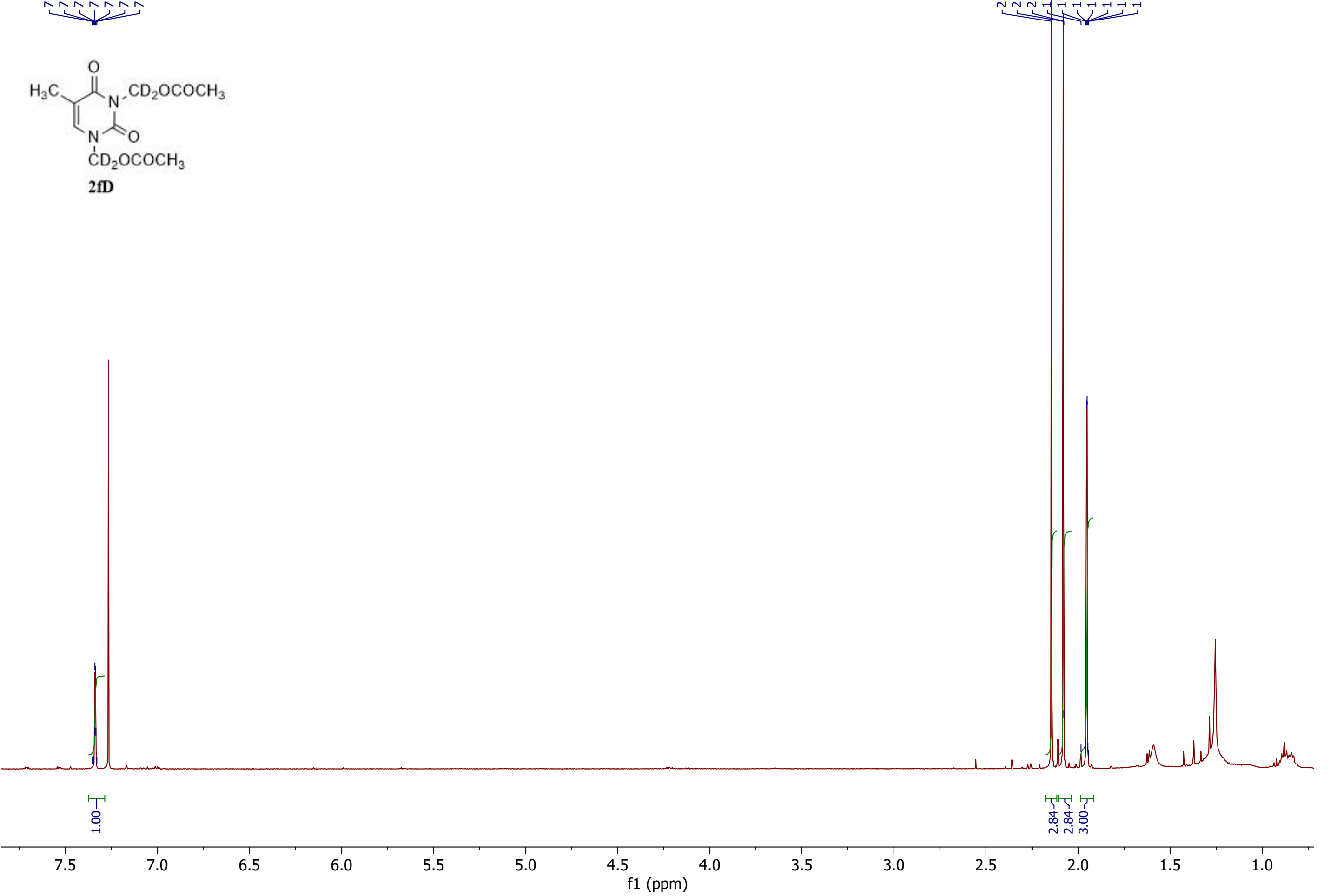
$13 \mathrm{C}\{1 \mathrm{H}\} \mathrm{NMR}, 125 \mathrm{MHz}, \mathrm{CDCl} 3$
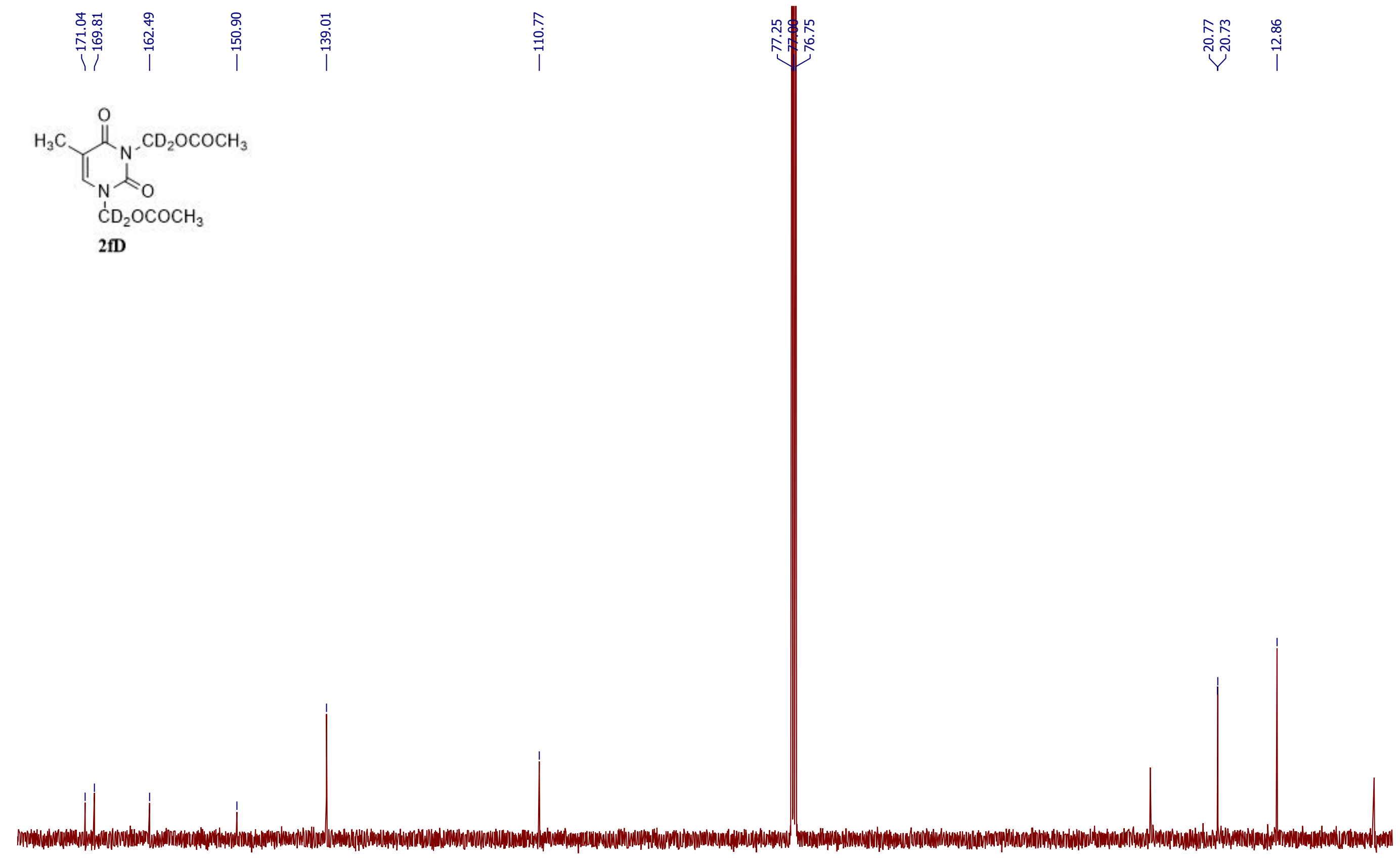
1H NMR, 600MHz, CDCl3
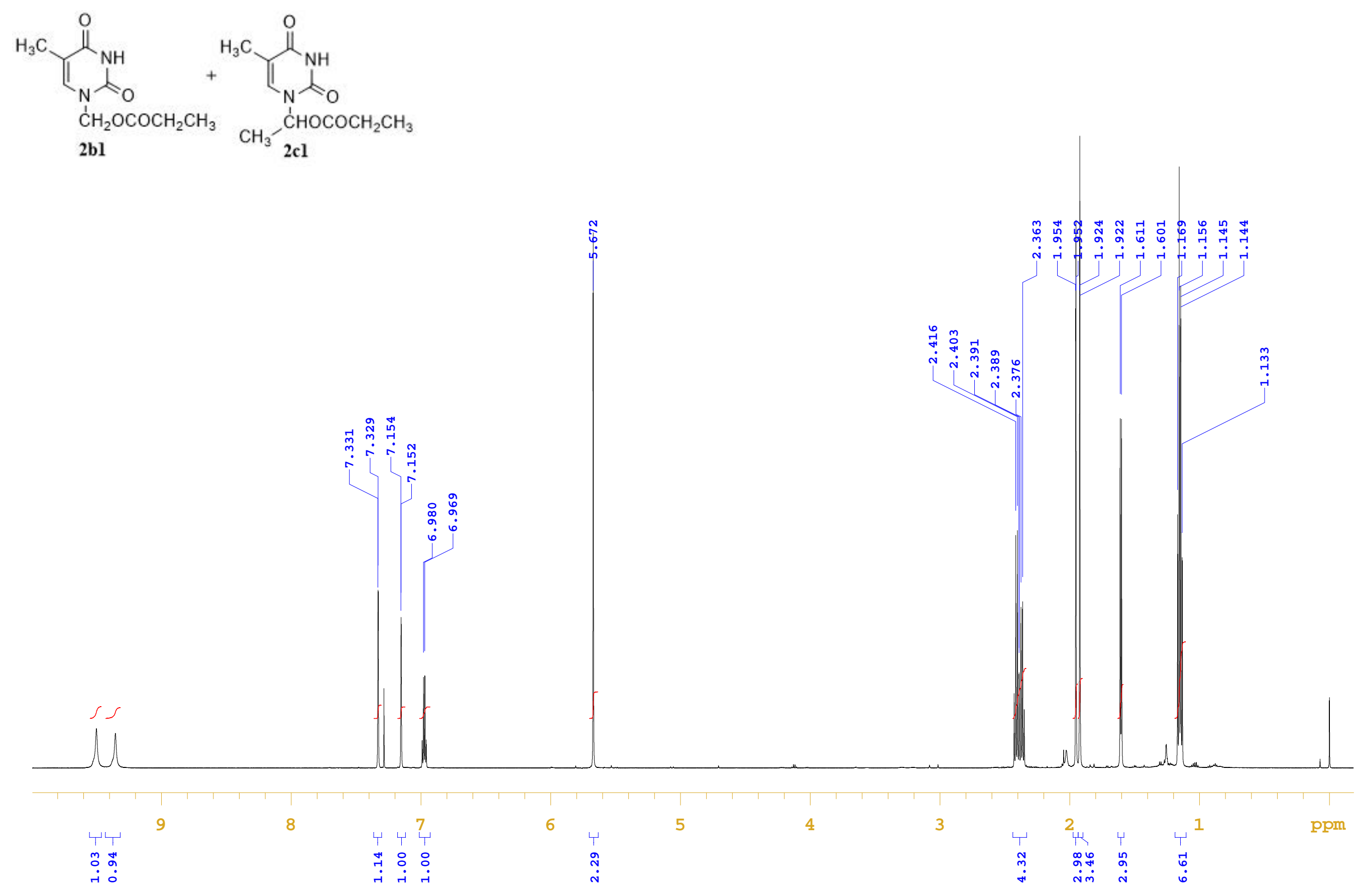
$13 \mathrm{C}\{1 \mathrm{H}\} \mathrm{NMR}, 150 \mathrm{MHz}, \mathrm{CDCl} 3$
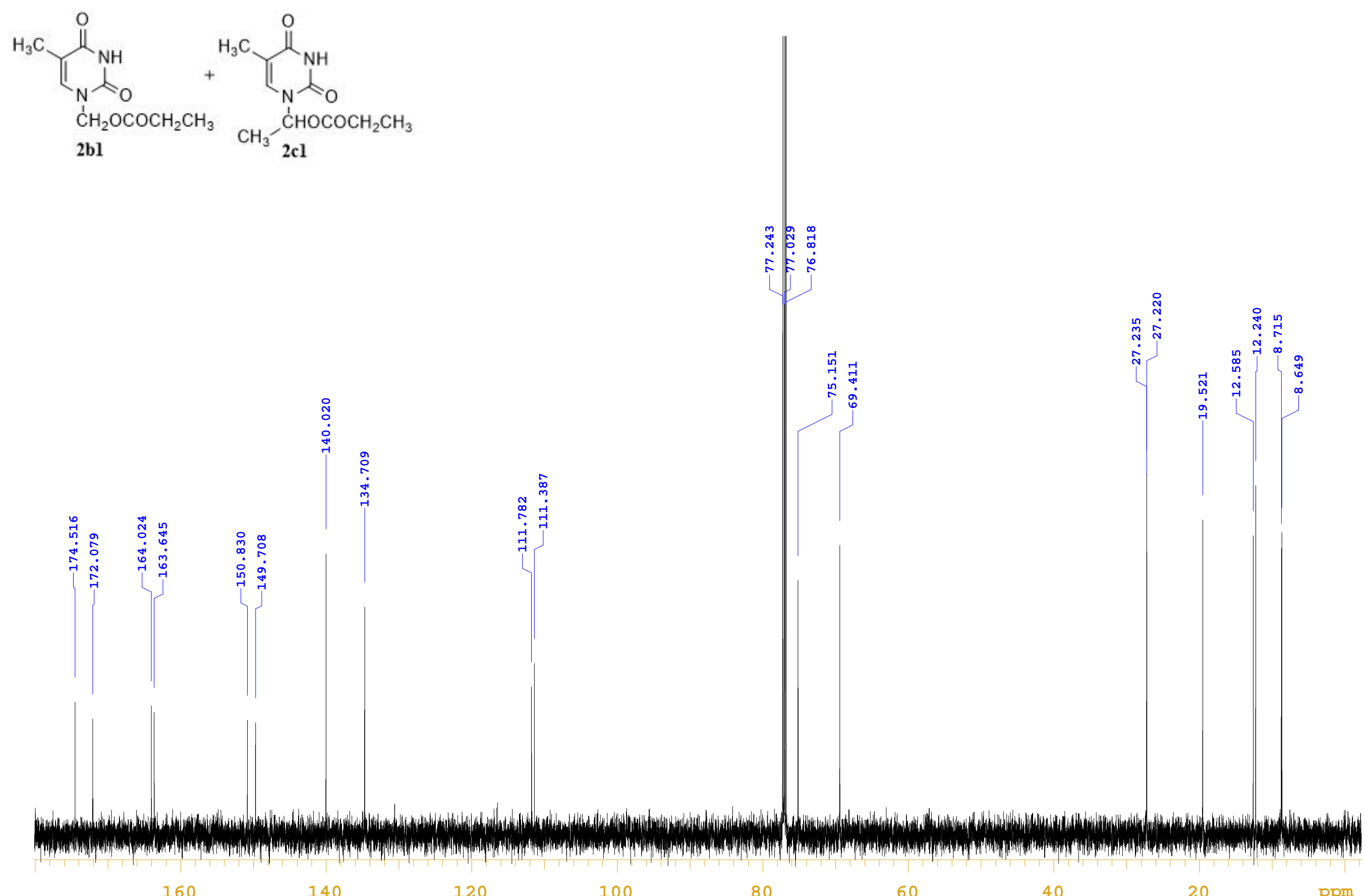

160

120

100

80

60

40

ppm 
1H NMR, 600MHz, CDCl3
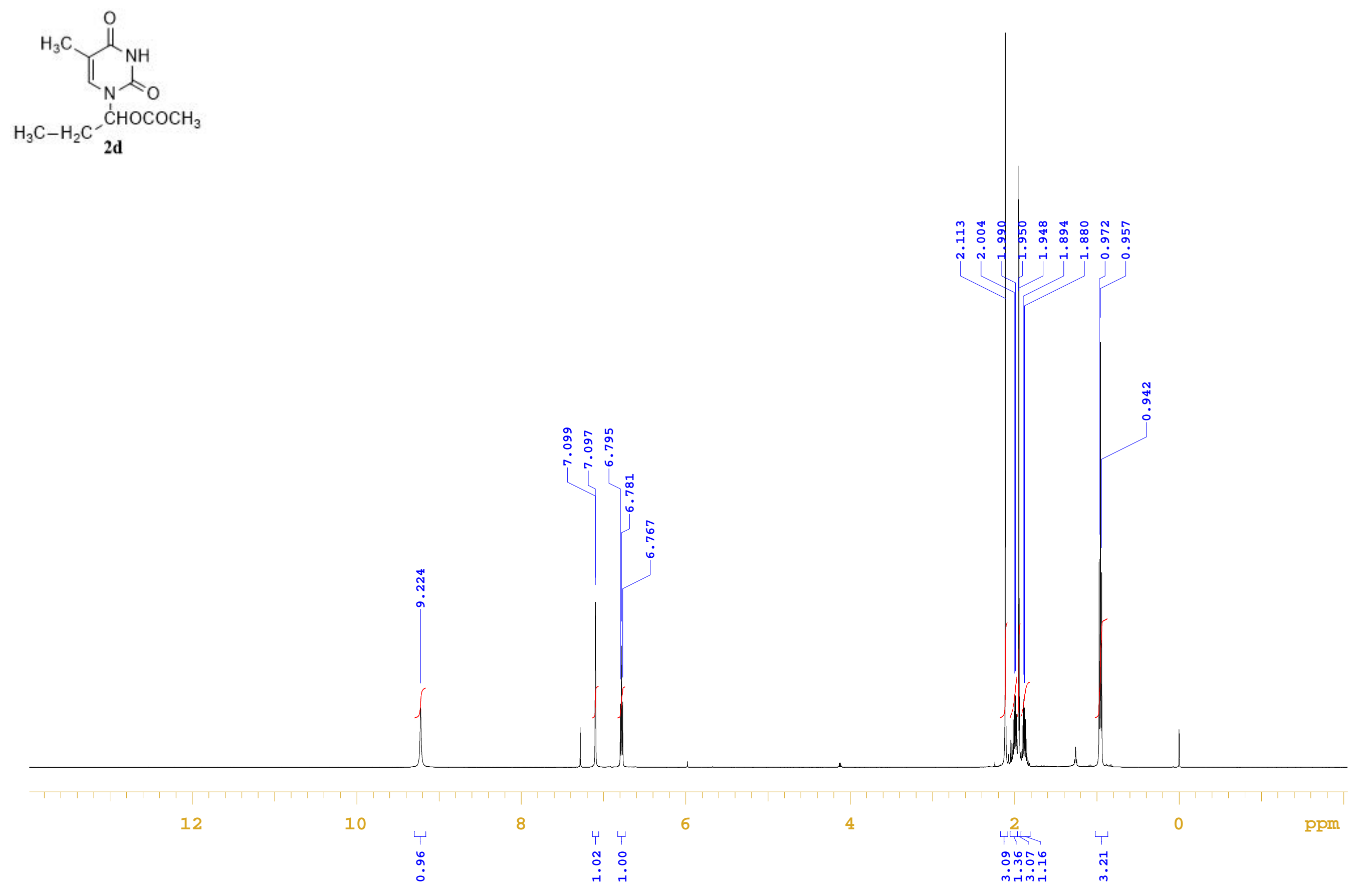
$13 \mathrm{C}\{1 \mathrm{H}\} \mathrm{NMR}, 150 \mathrm{MHz}, \mathrm{CDCl} 3$
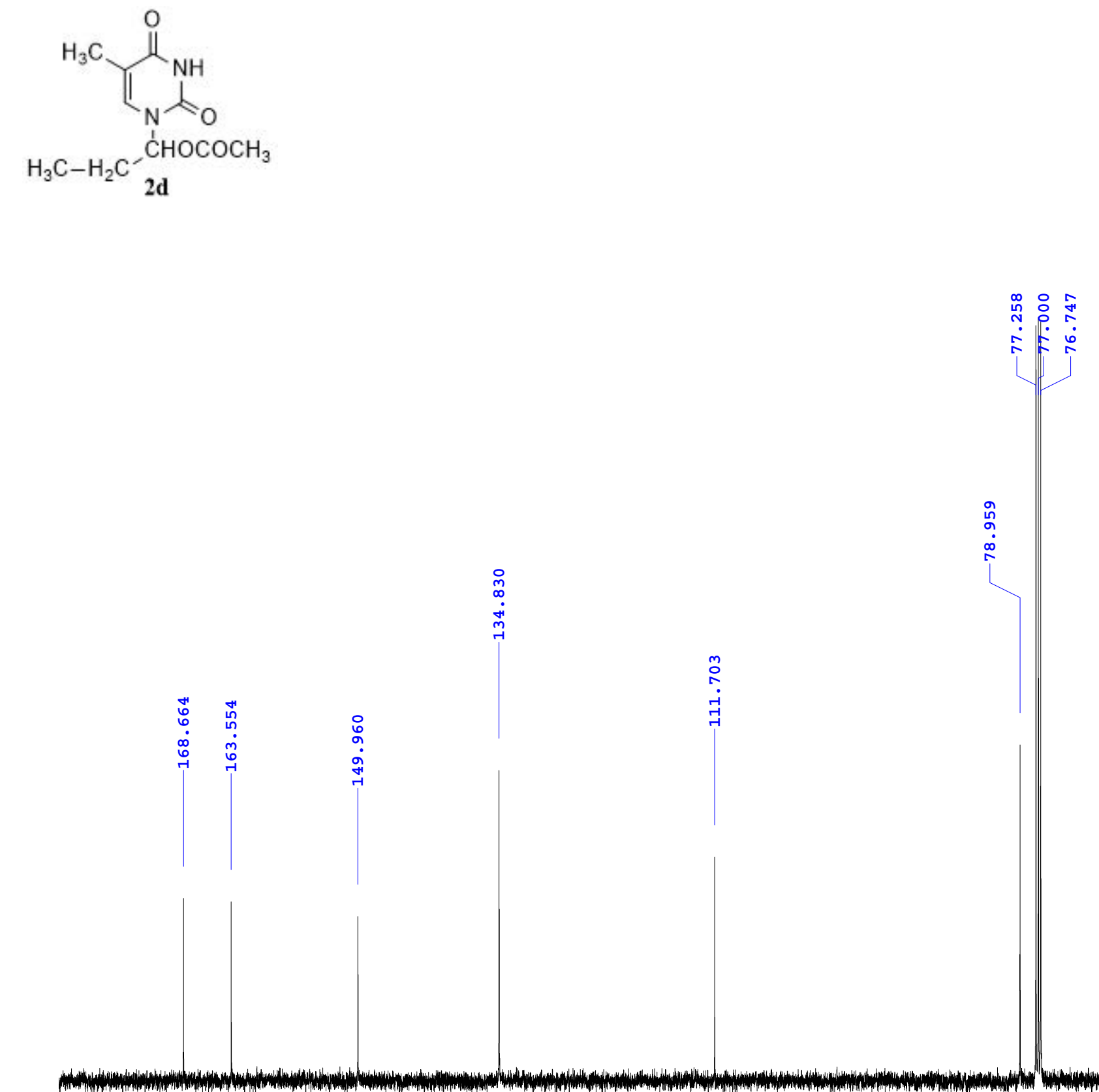

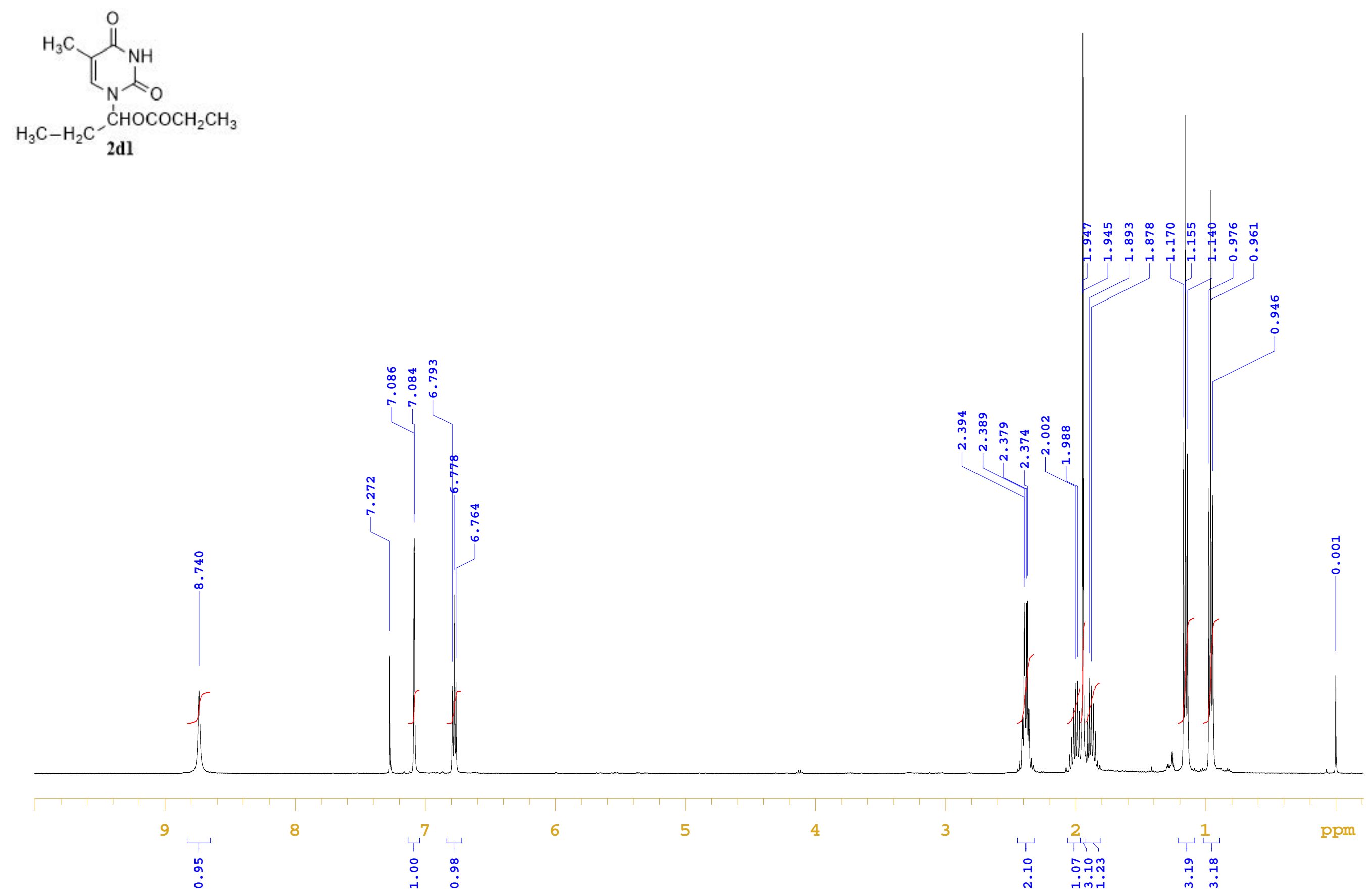
$13 \mathrm{C}\{1 \mathrm{H}\} \mathrm{NMR}, 150 \mathrm{MHz}, \mathrm{CDCl} 3$
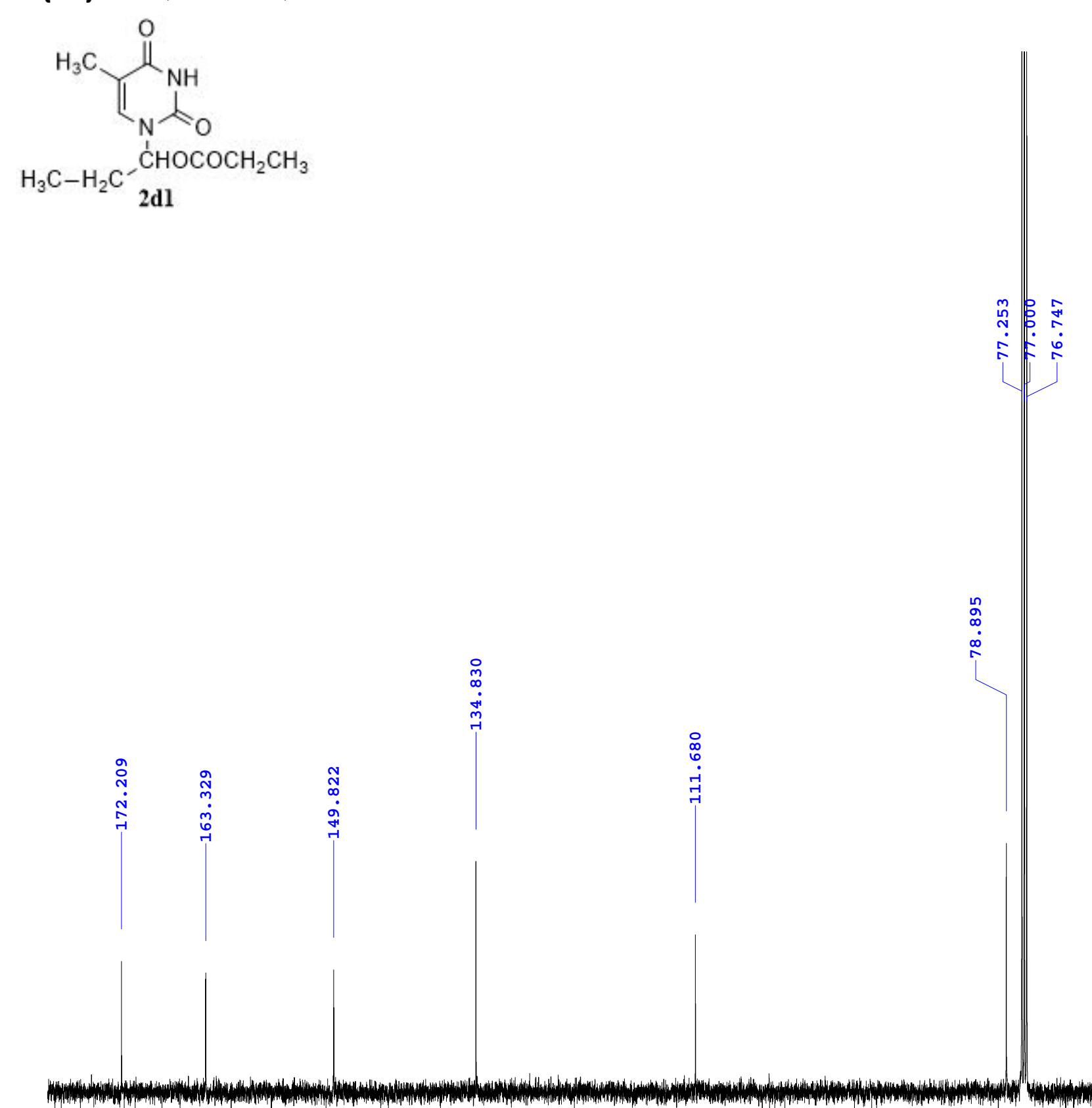

พี

ก

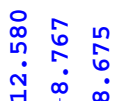

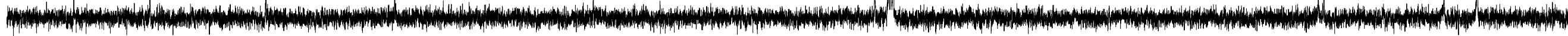





\section{$13 \mathrm{C}\{1 \mathrm{H}\} \mathrm{NMR}, 150 \mathrm{MHz}, \mathrm{CDCl} 3$}

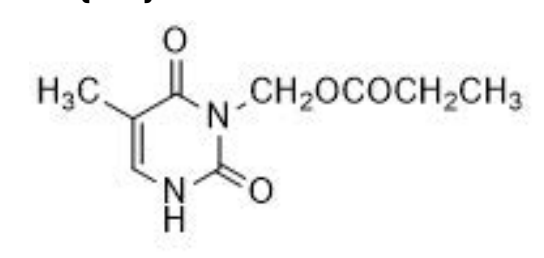

2el

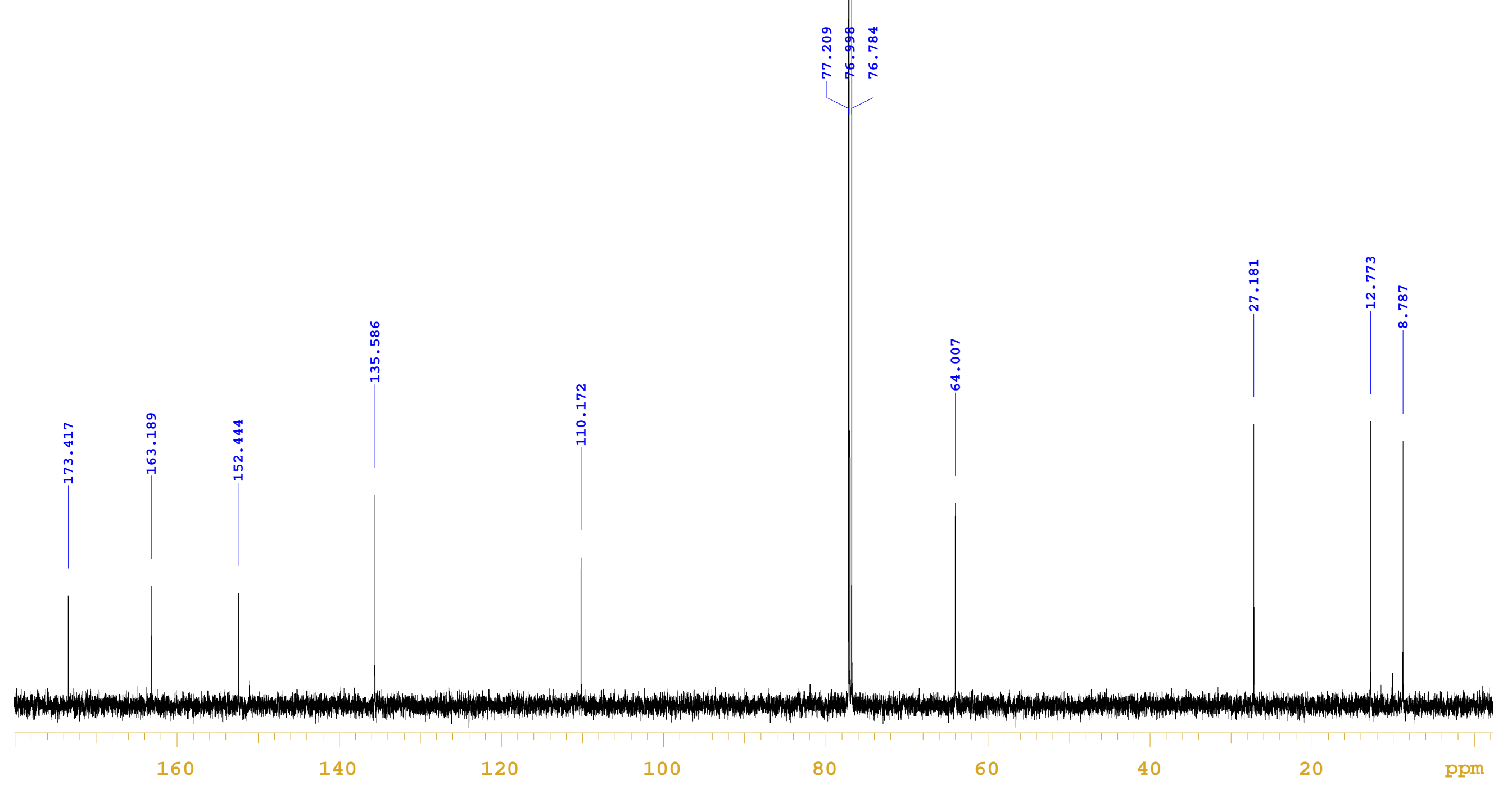


1H NMR, 600MHz, CDCl3
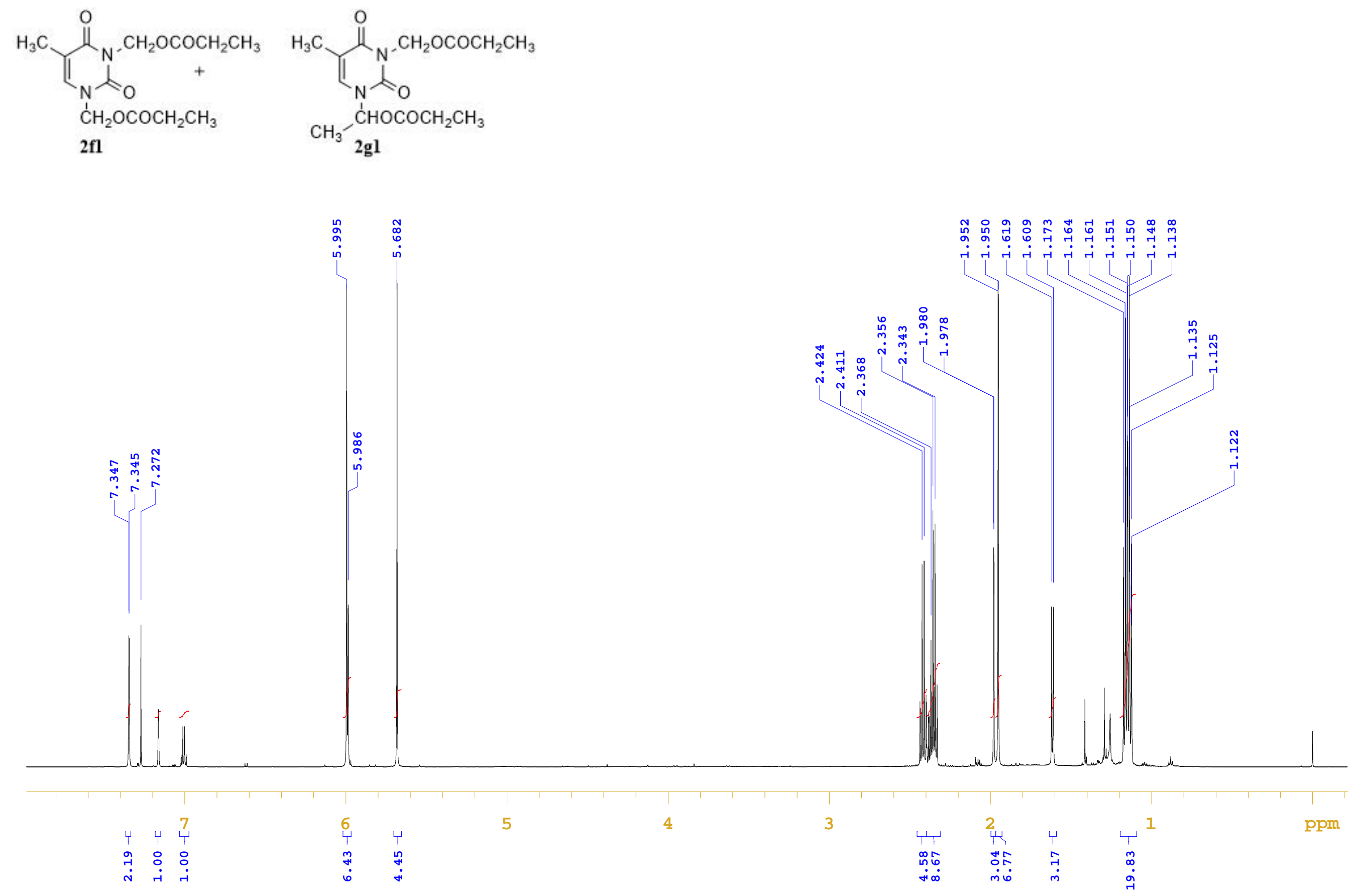
$13 \mathrm{C}\{1 \mathrm{H}\} \mathrm{NMR}, 150 \mathrm{MHz}, \mathrm{CDCl} 3$

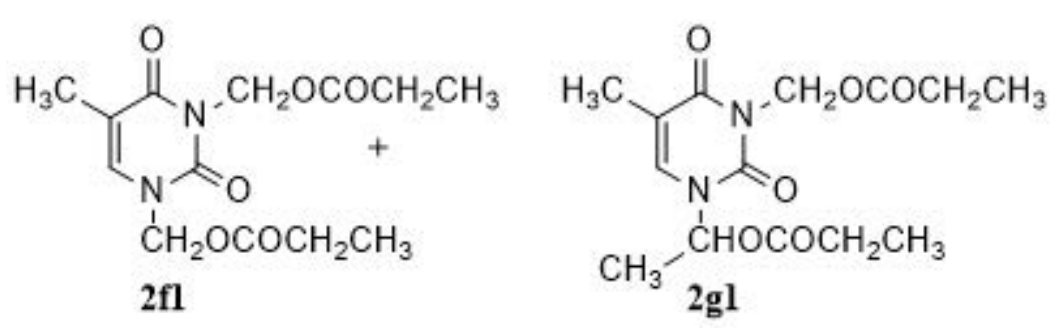

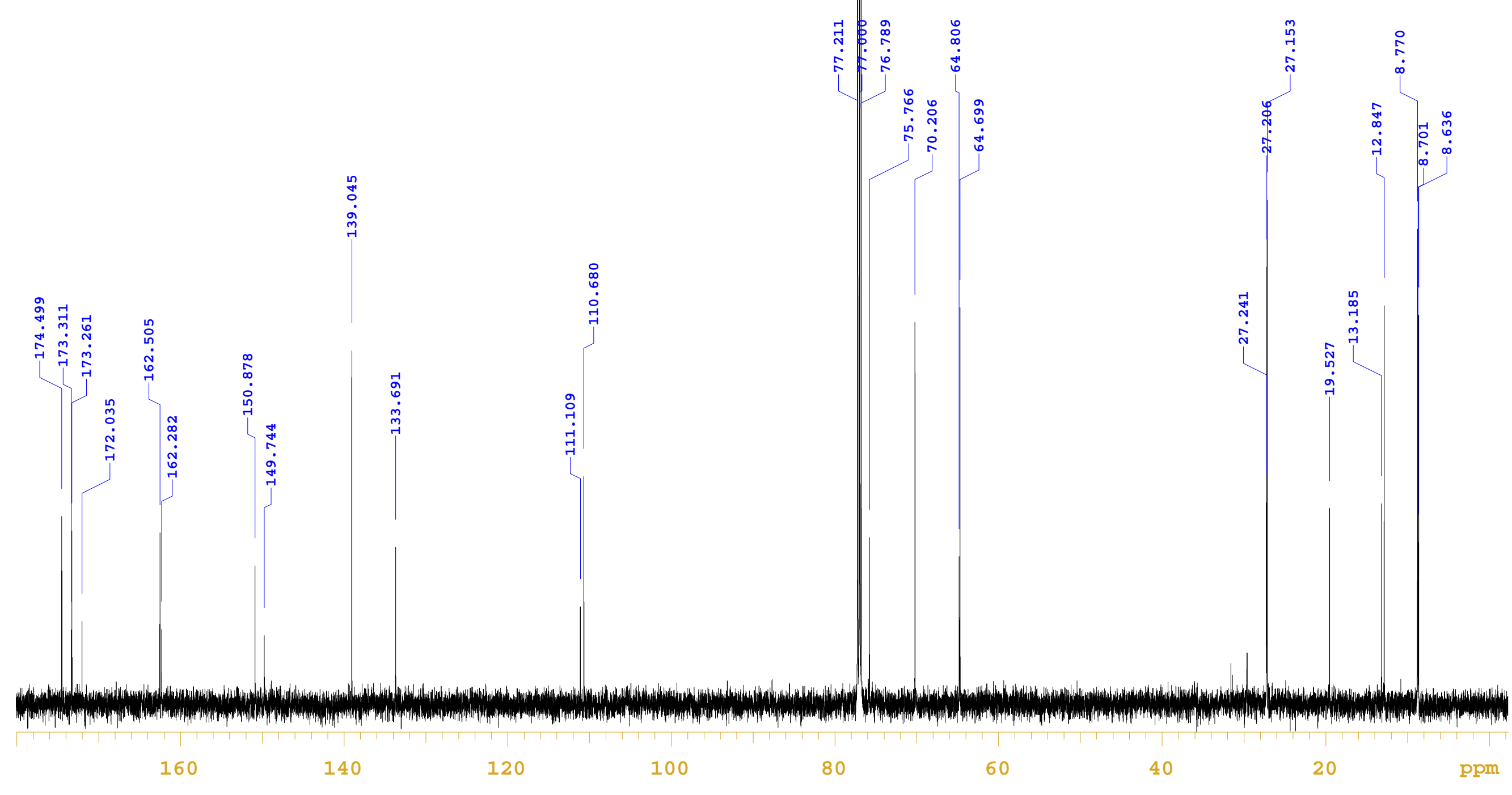


1H NMR, 600MHz, CDCl3

$$
\mathrm{H}_{3} \mathrm{C}-\mathrm{H}_{2} \mathrm{C}_{2 \mathrm{~h}}^{\mathrm{C}}
$$

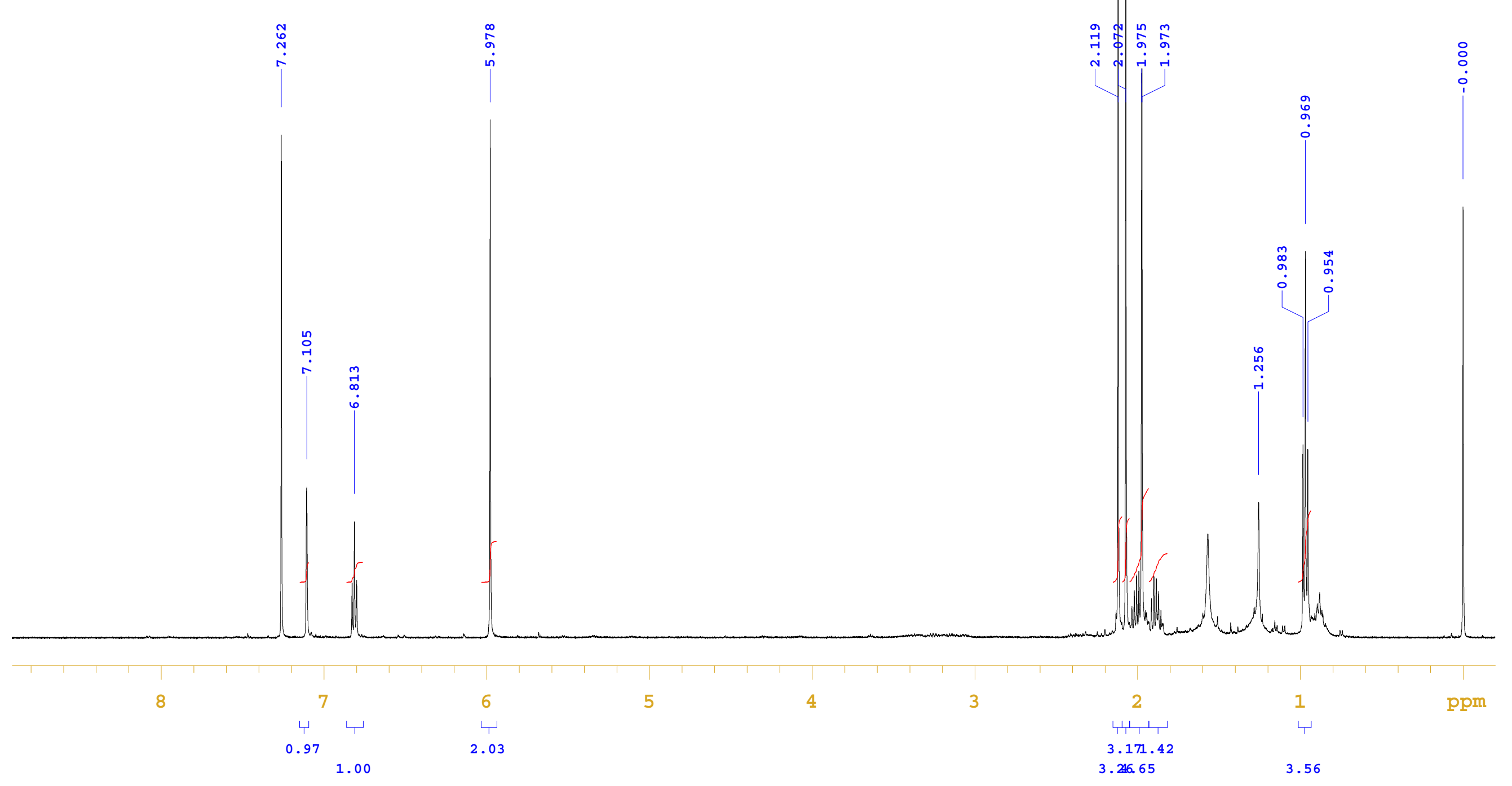


13C $\{1 \mathrm{H}\} \mathrm{NMR}, 150 \mathrm{MHz}, \mathrm{CDCl} 3$
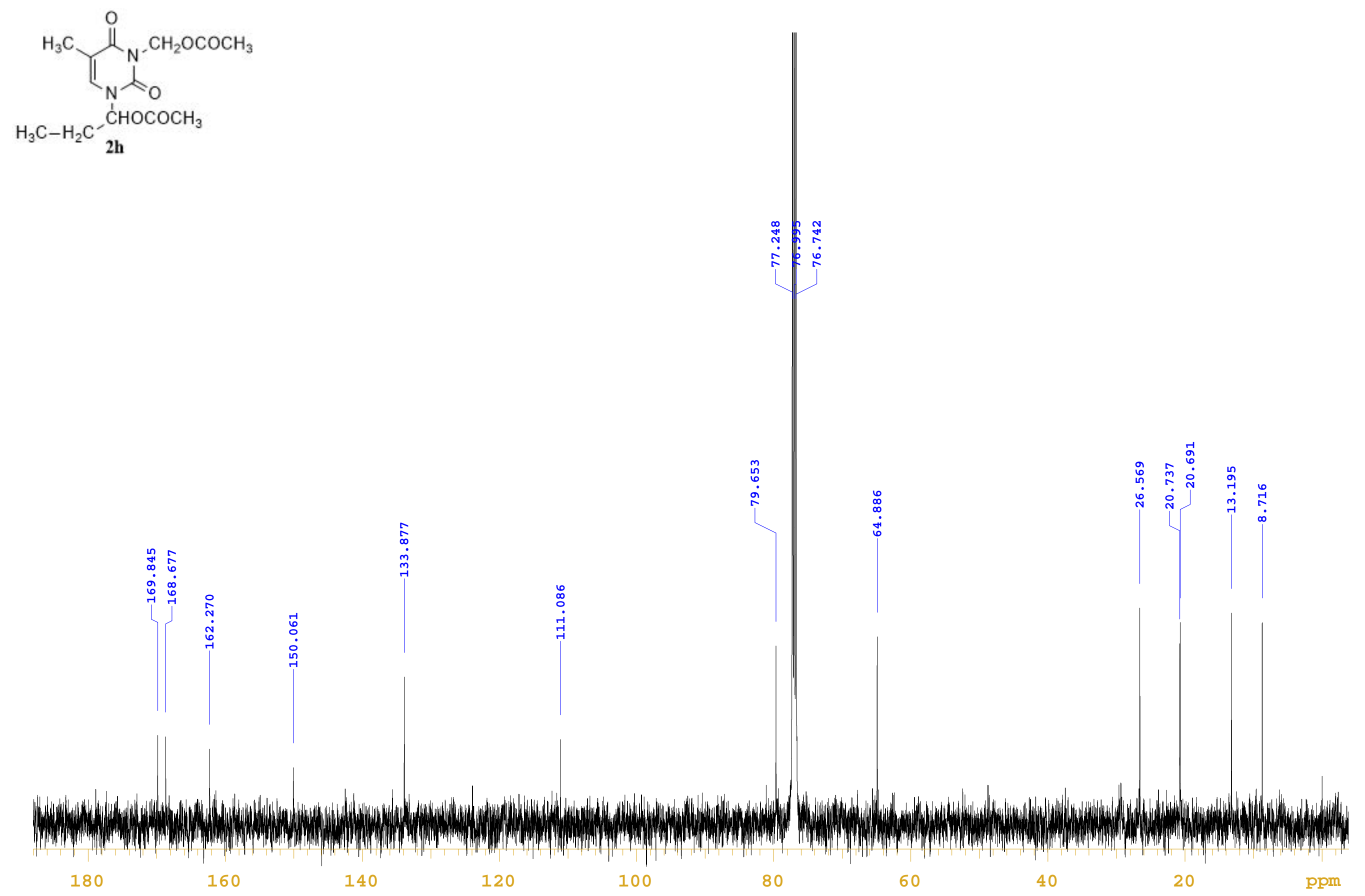


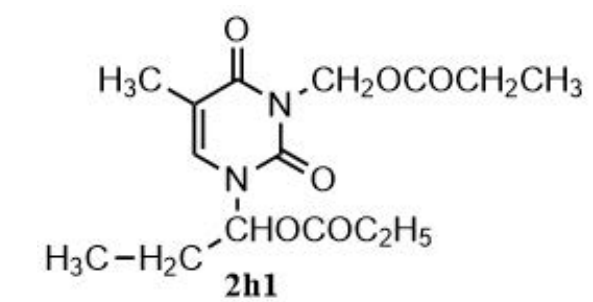

苟苇
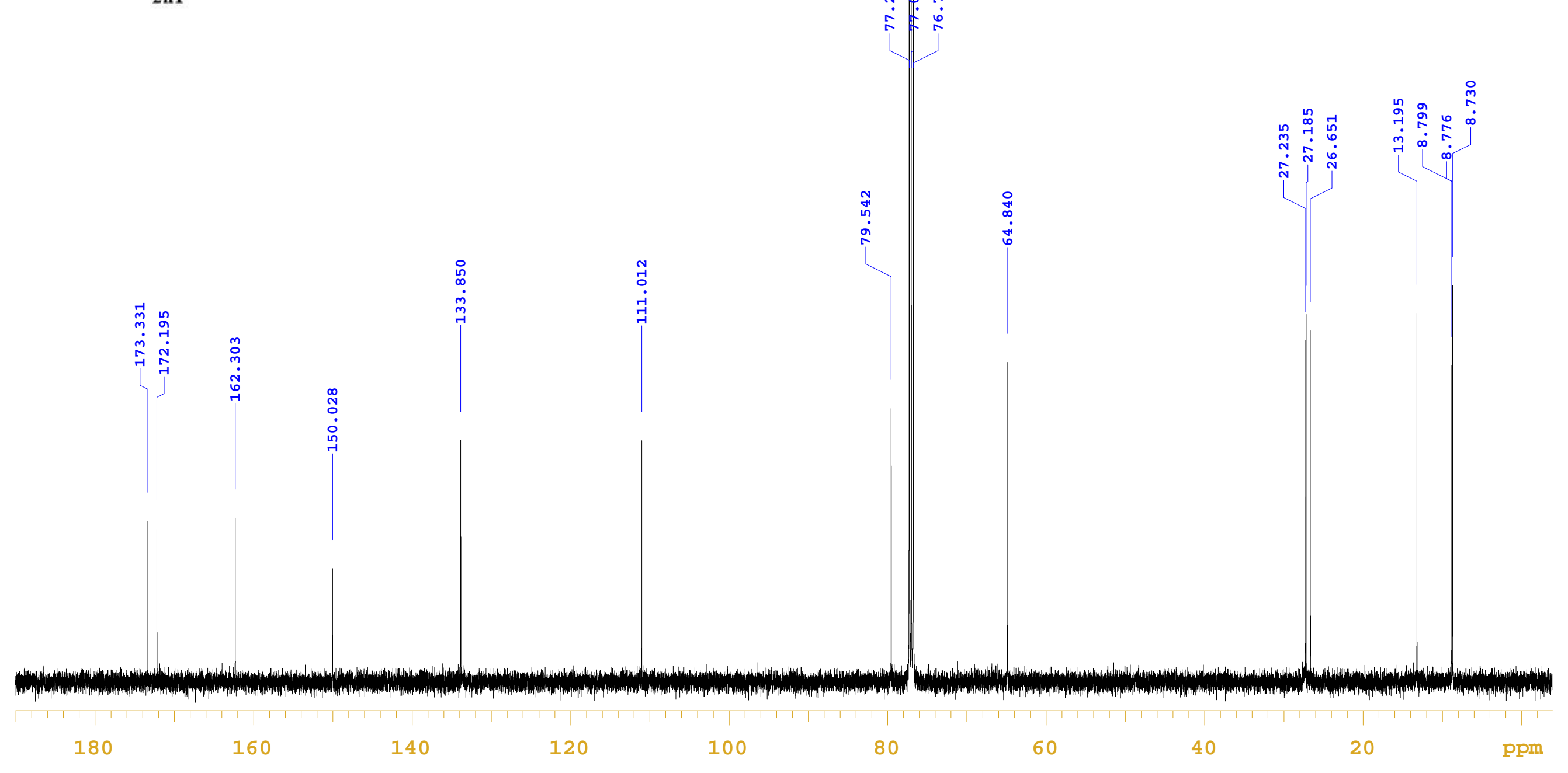


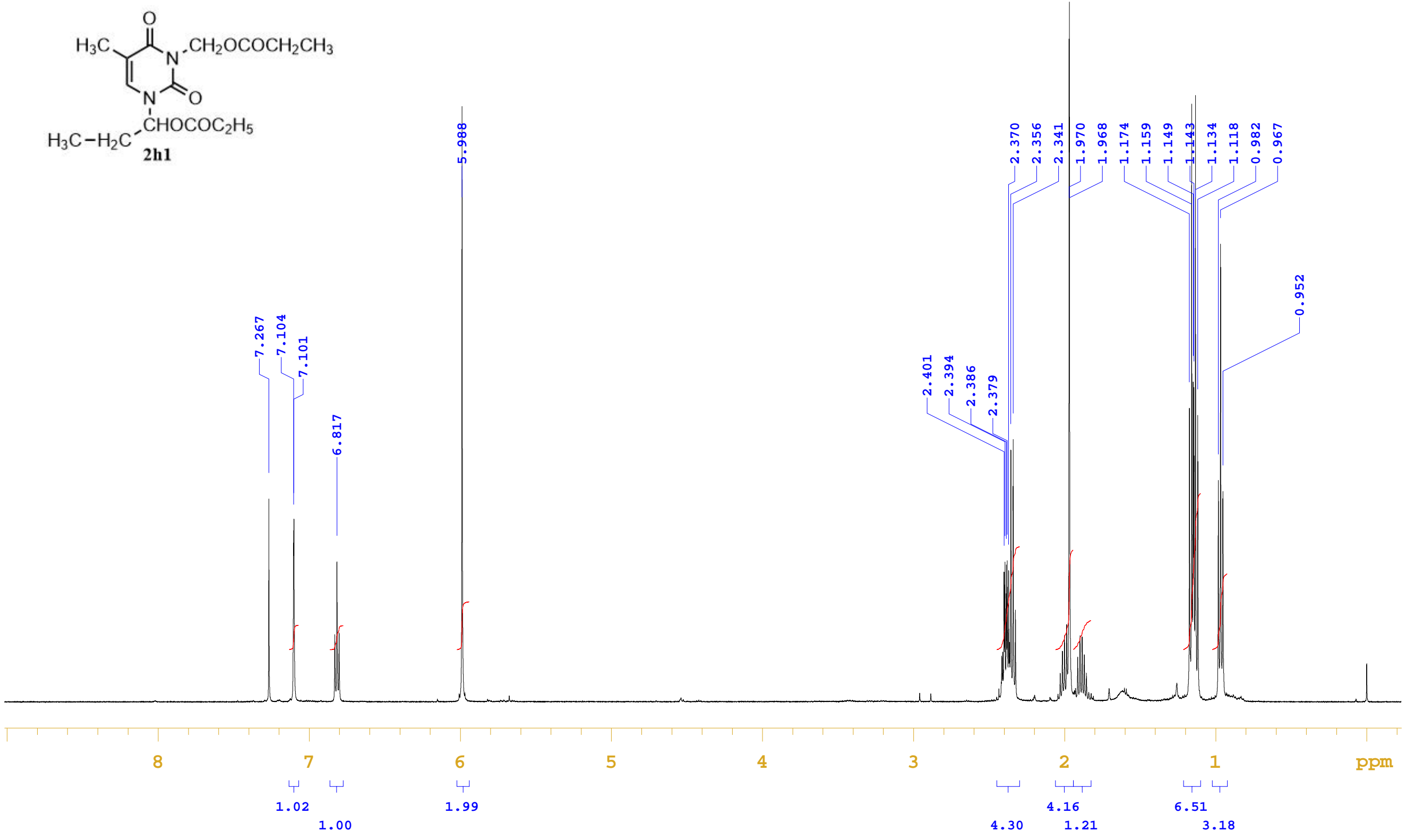



$13 \mathrm{C}\{1 \mathrm{H}\} \mathrm{NMR}, 150 \mathrm{MHz}, \mathrm{CDCl} 3$

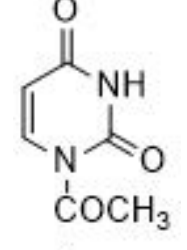

3a

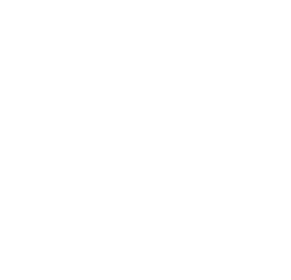

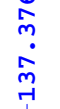

مُ

-

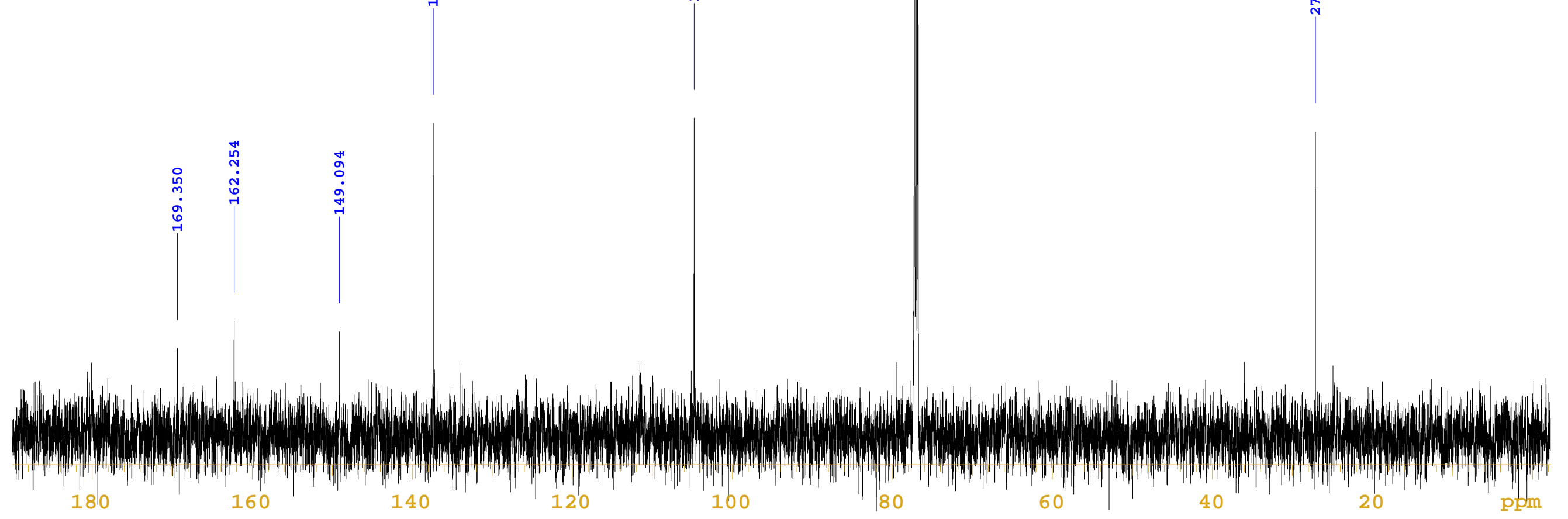


1H NMR, $600 \mathrm{MHz}, \mathrm{CDCl} 3$
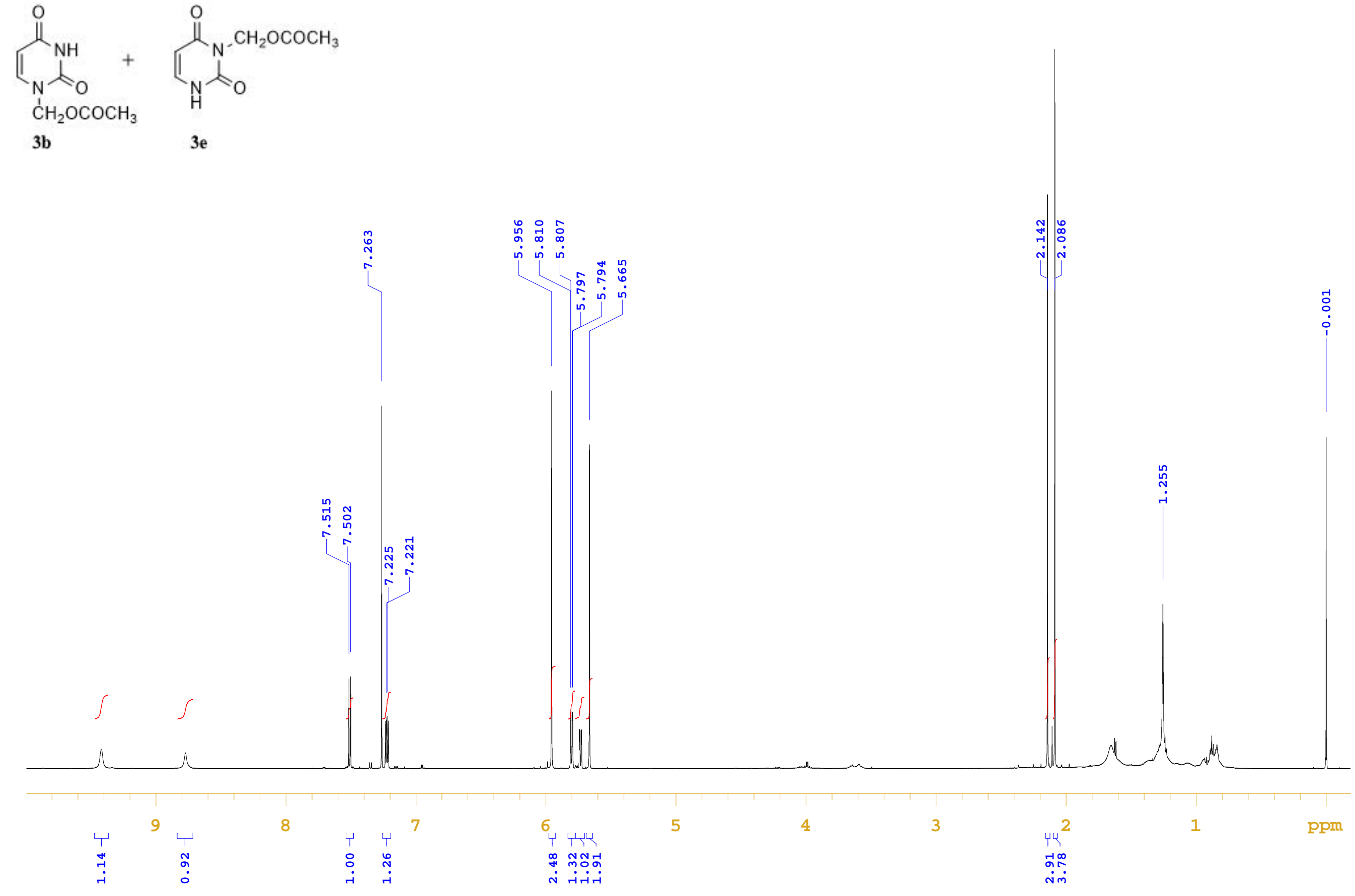
$13 \mathrm{C}\{1 \mathrm{H}\} \mathrm{NMR}, 150 \mathrm{MHz}, \mathrm{CDCl} 3$
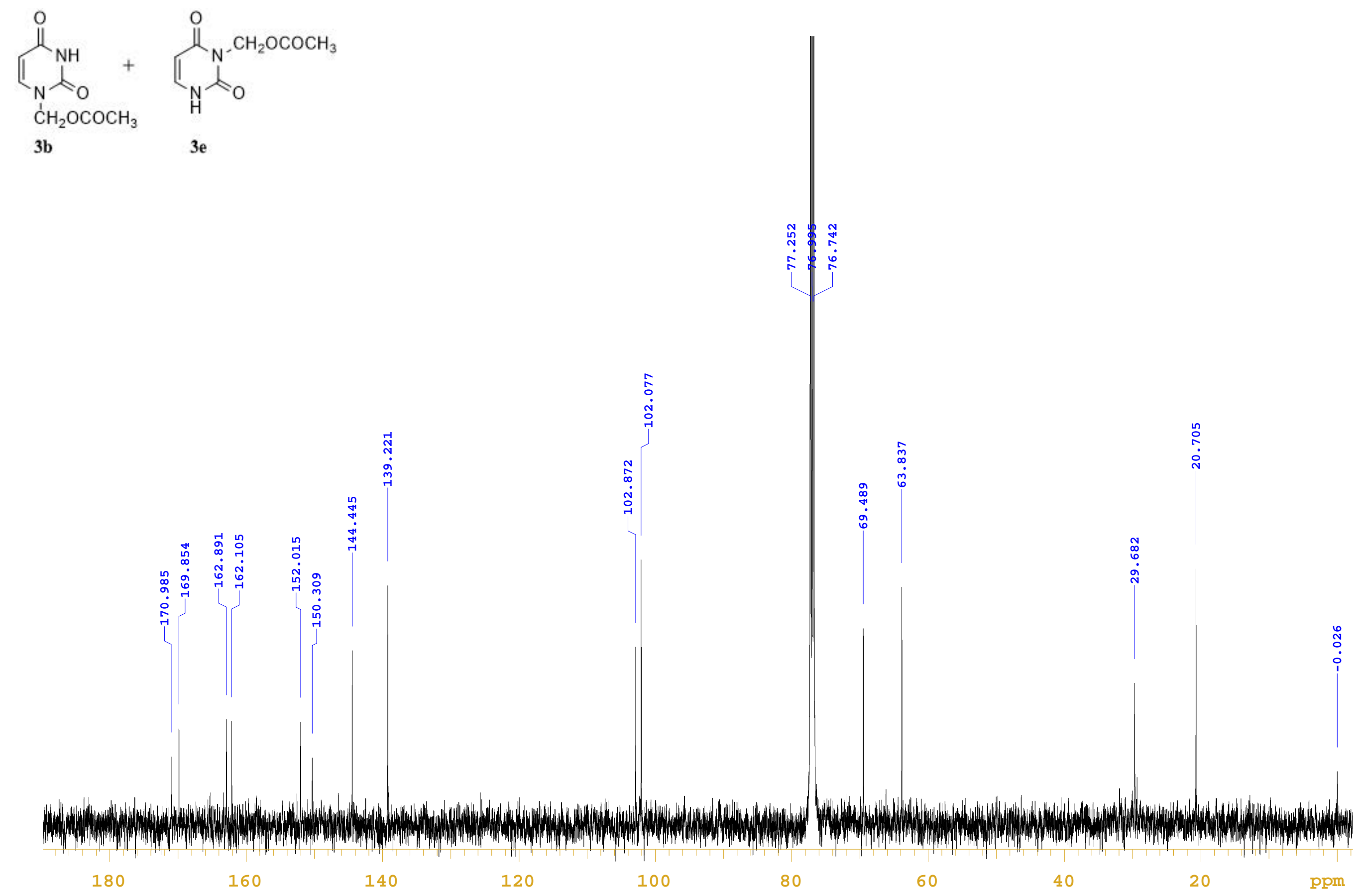

$13 \mathrm{C}\{1 \mathrm{H}\} \mathrm{NMR}, 150 \mathrm{MHz}, \mathrm{CDCl} 3$
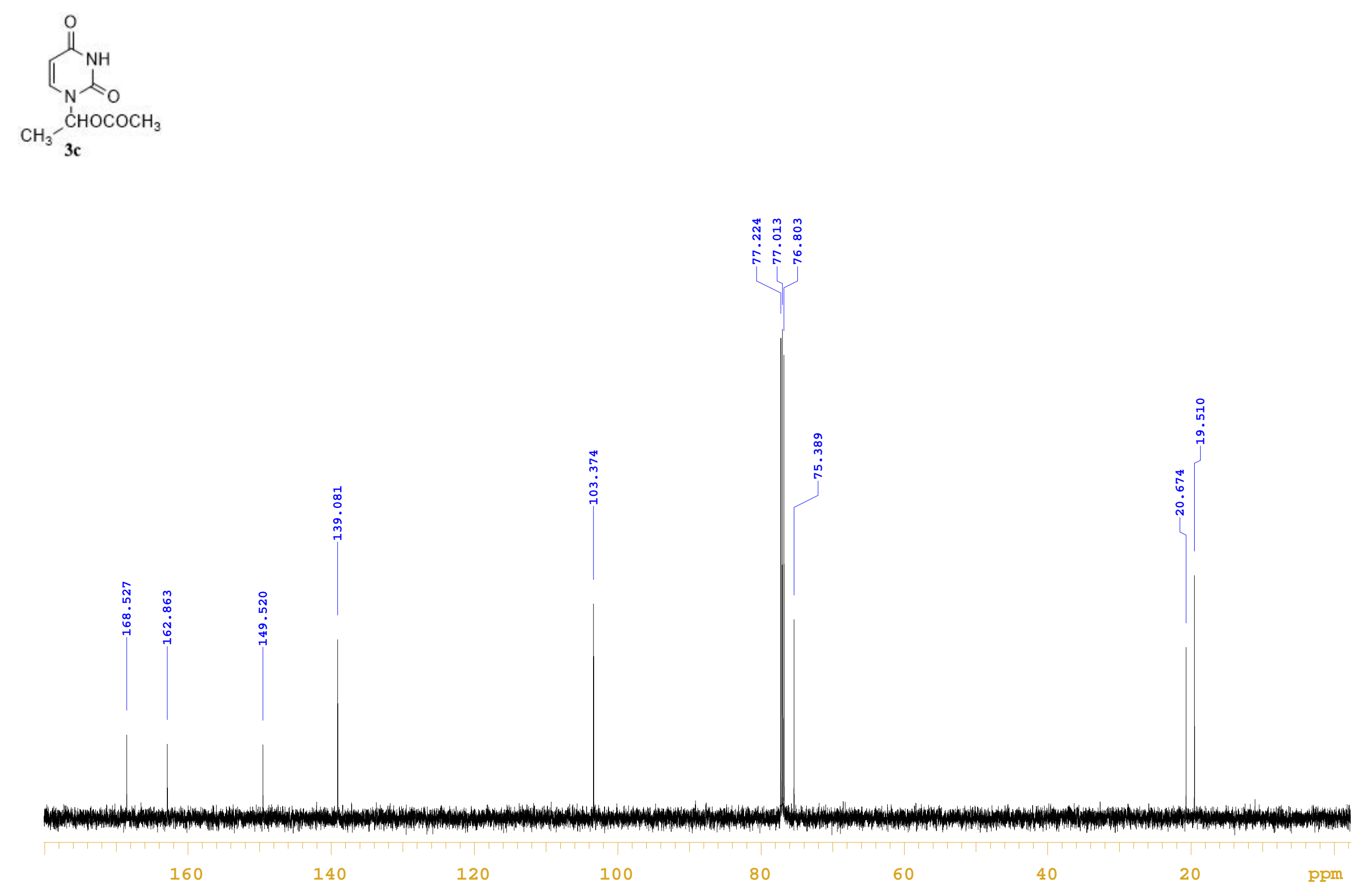
1H NMR, 500MHz, CDCl3

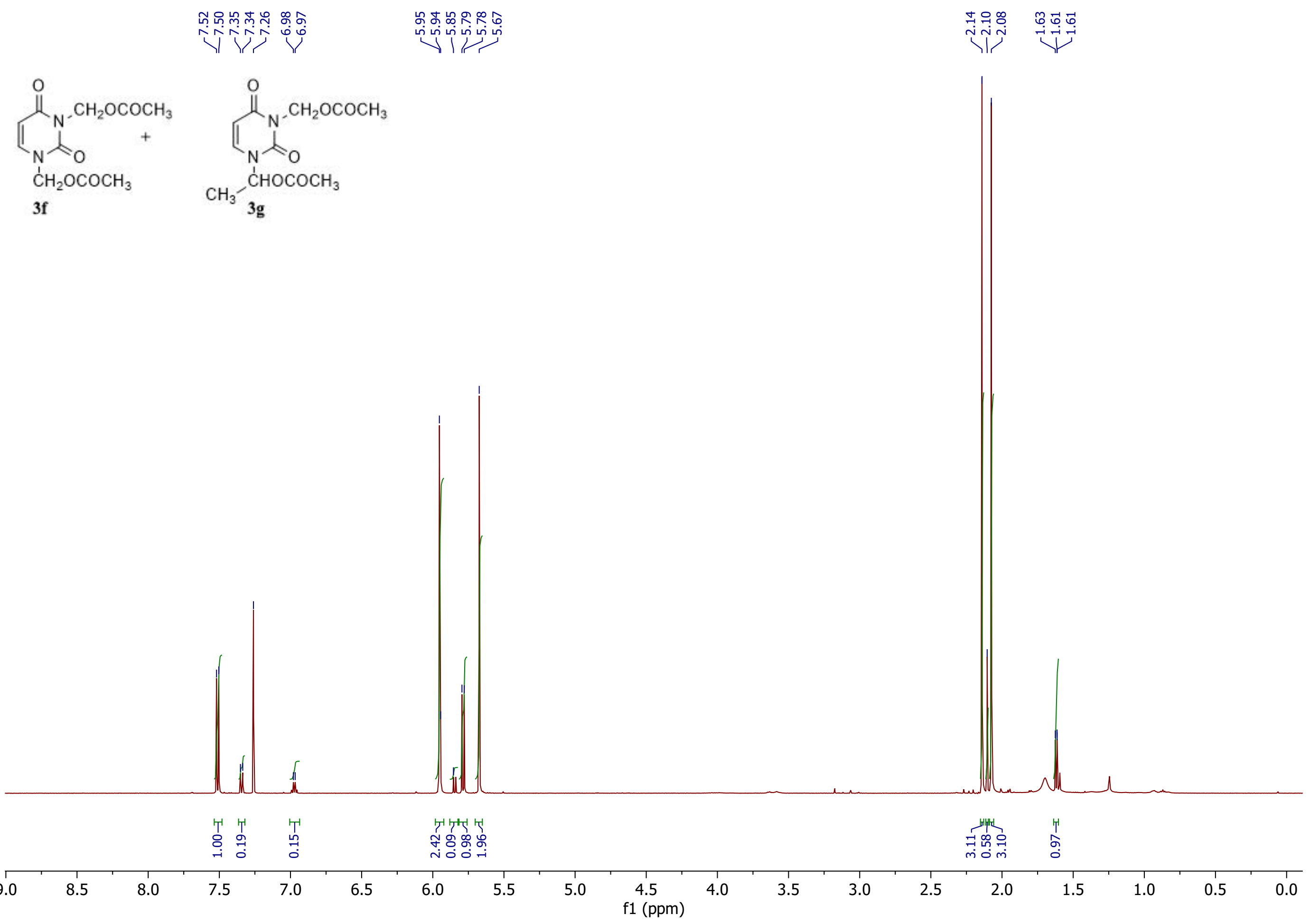


$13 \mathrm{C}\{1 \mathrm{H}\} \mathrm{NMR}, 125 \mathrm{MHz}, \mathrm{CDCl} 3$

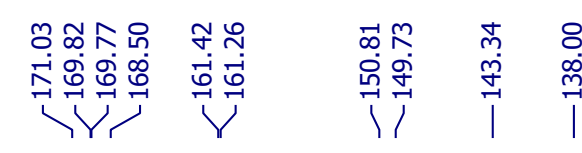<smiles></smiles>
$3 f$<smiles>CC(=O)Cn1c(=O)ccn(COC(C)C)c1=O</smiles>

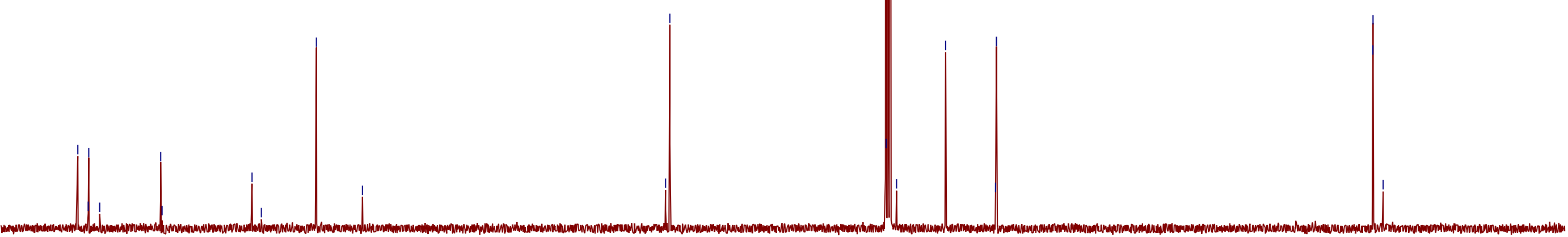


1H NMR, $600 \mathrm{MHz}, \mathrm{CDCl} 3$

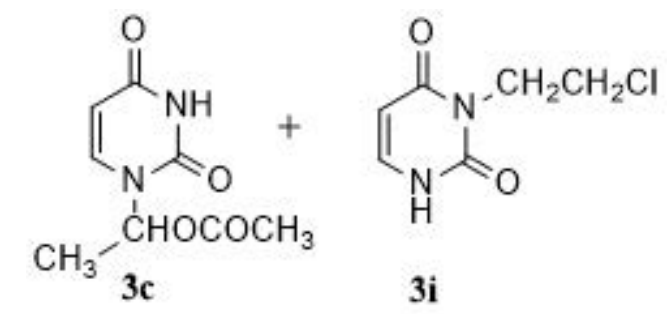

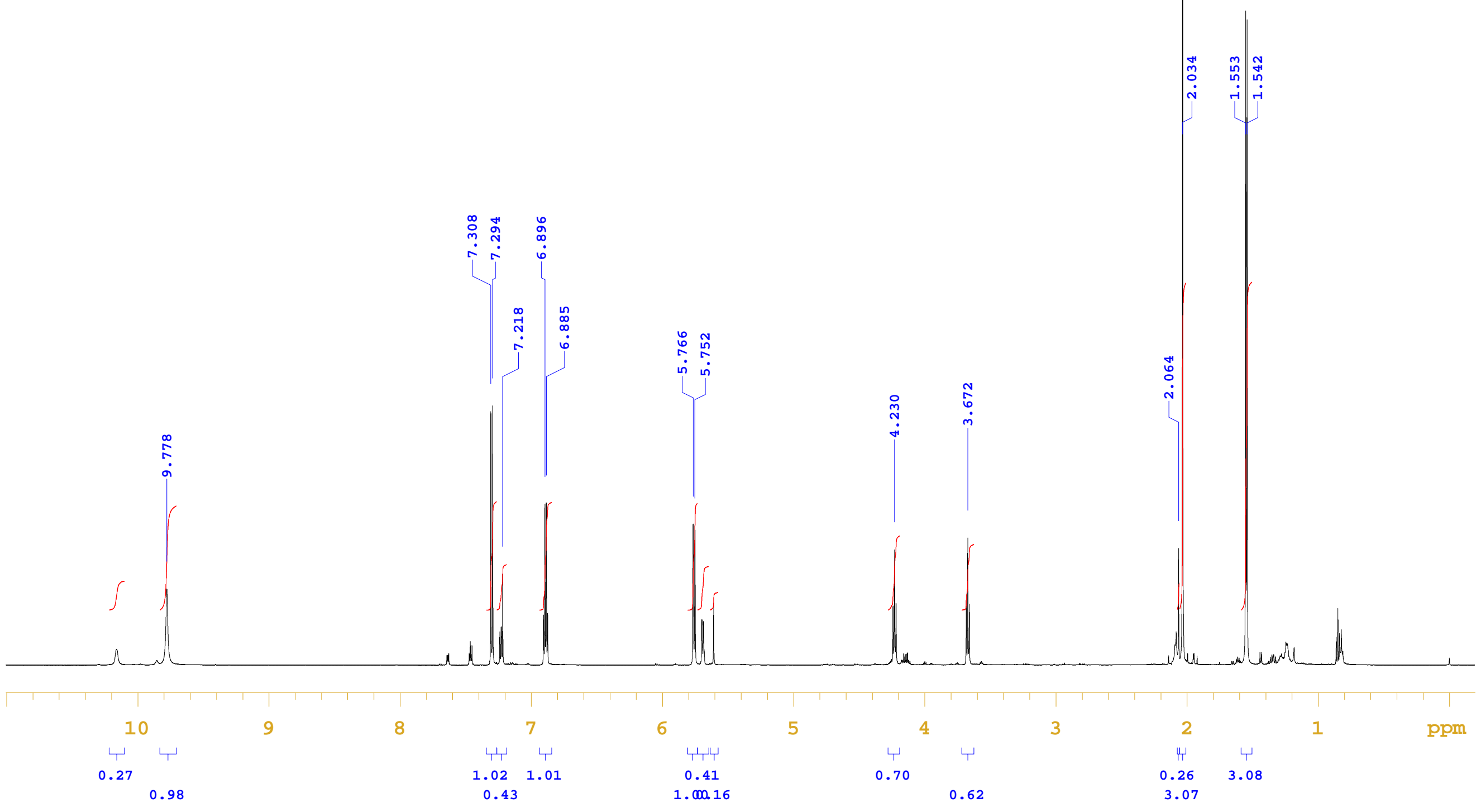


$13 \mathrm{C}\{1 \mathrm{H}\} \mathrm{NMR}, 150 \mathrm{MHz}, \mathrm{CDCl} 3$
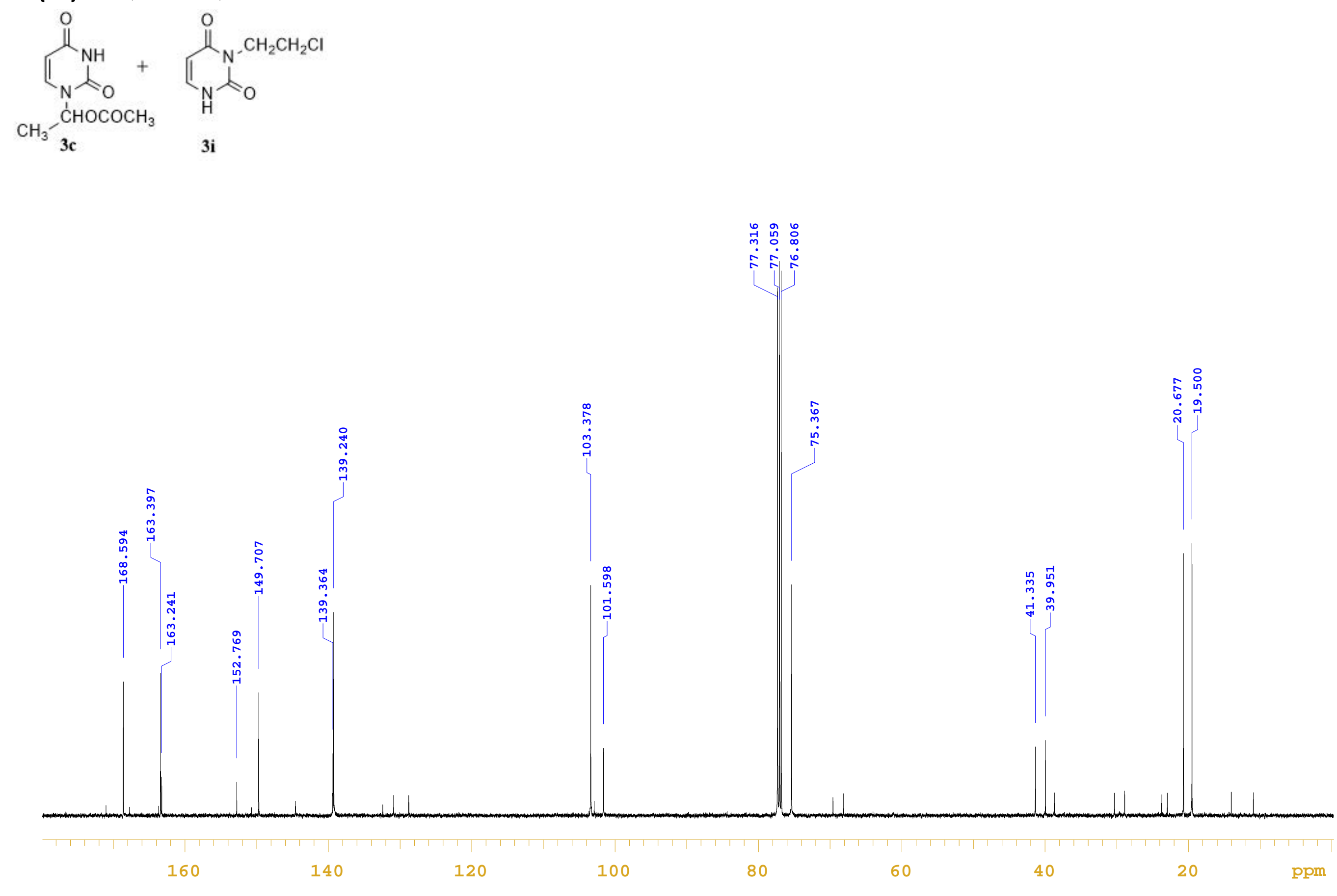
1H NMR, 600MHz, CDCl3
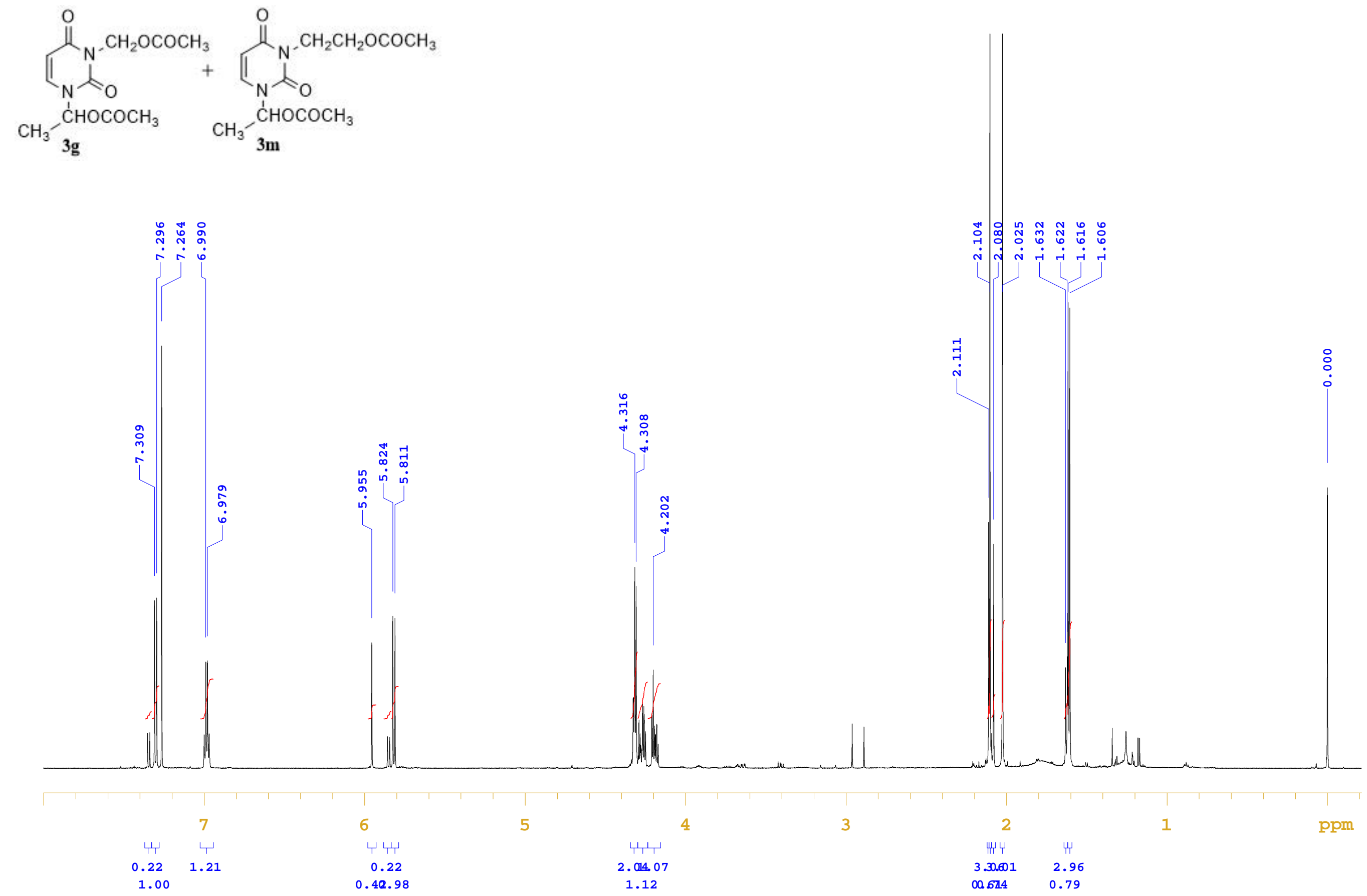
$13 \mathrm{C}\{1 \mathrm{H}\} \mathrm{NMR}, 150 \mathrm{MHz}, \mathrm{CDCl} 3$
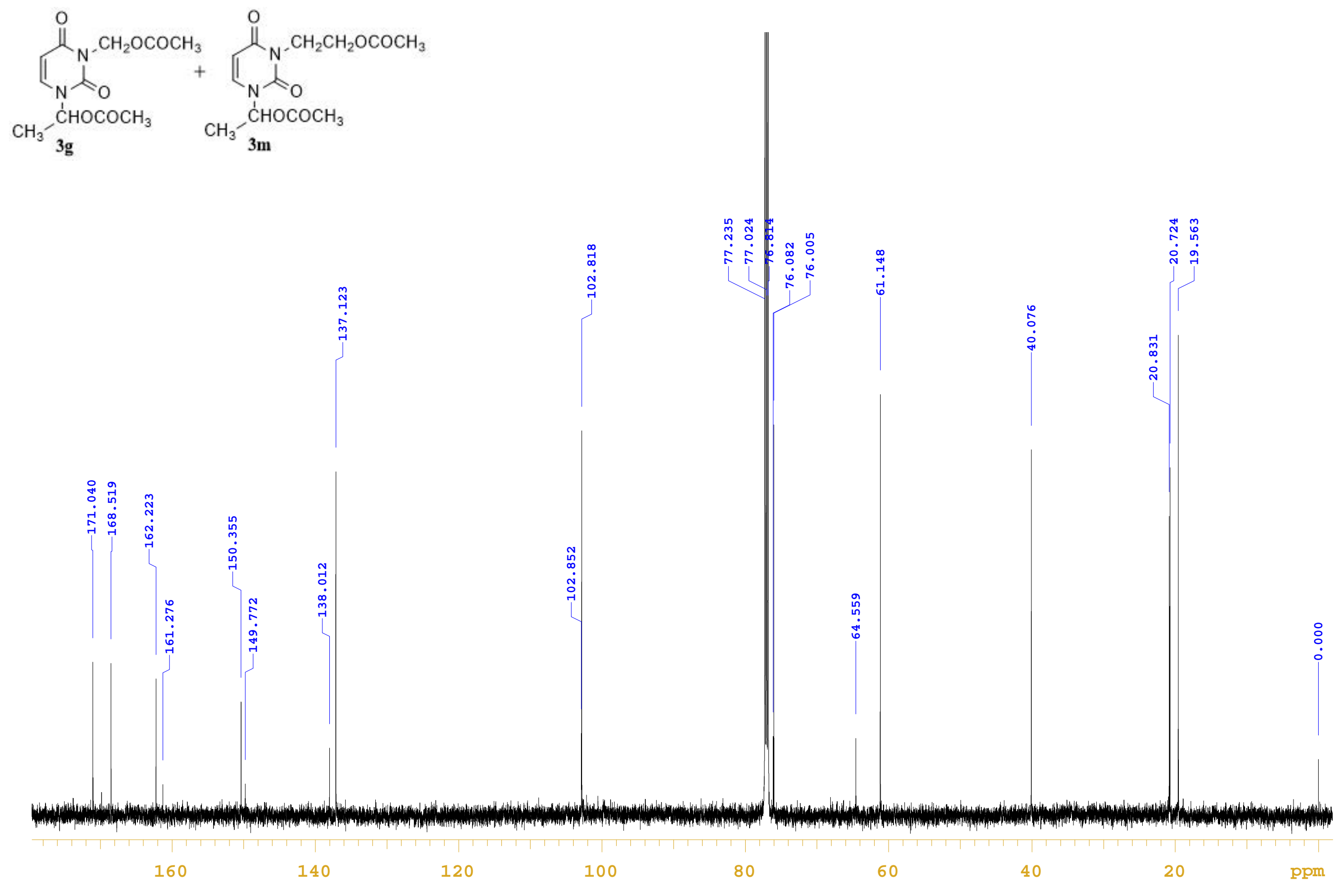

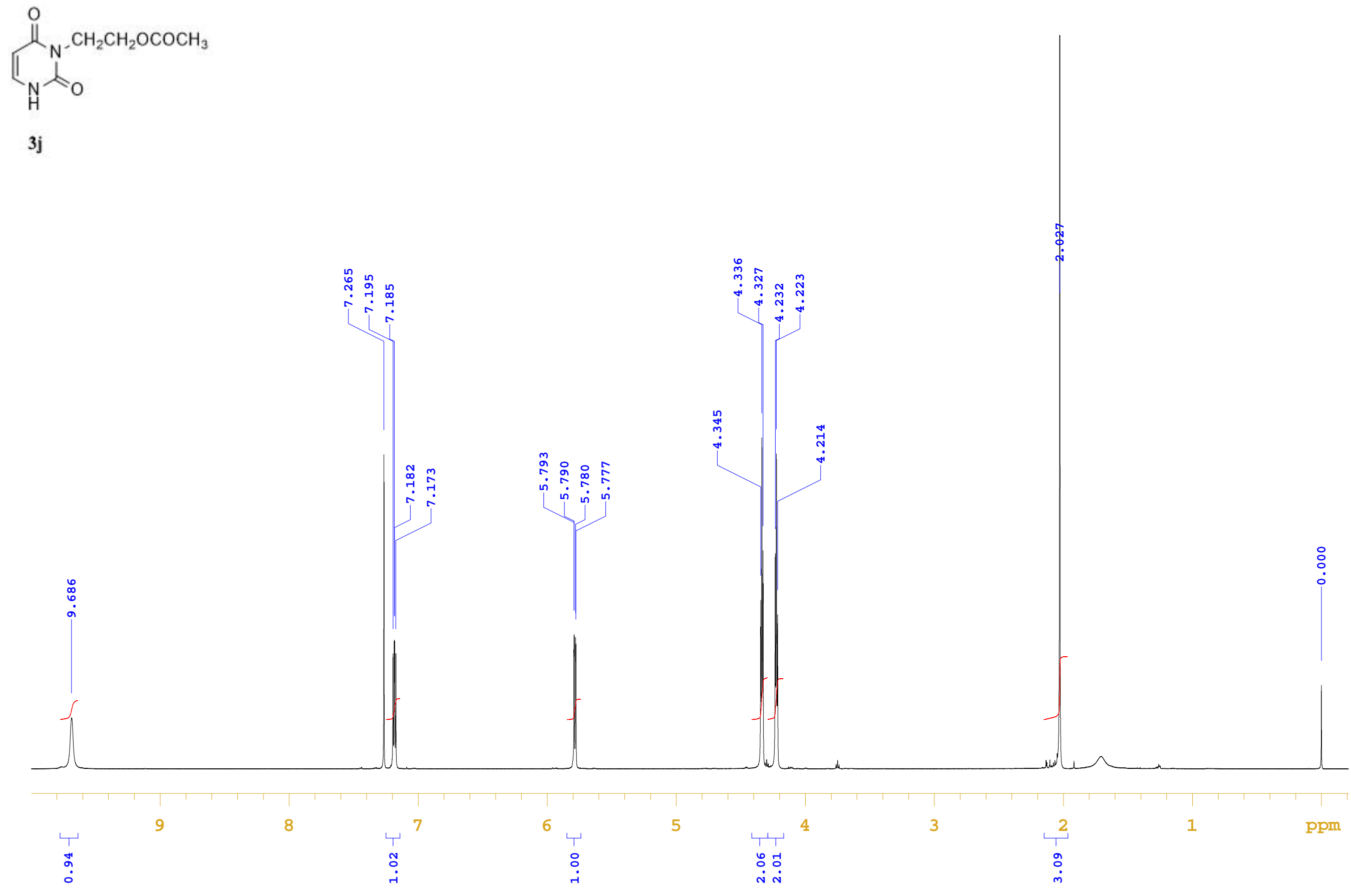
$13 \mathrm{C}\{1 \mathrm{H}\} \mathrm{NMR}, 150 \mathrm{MHz}, \mathrm{CDCl} 3$

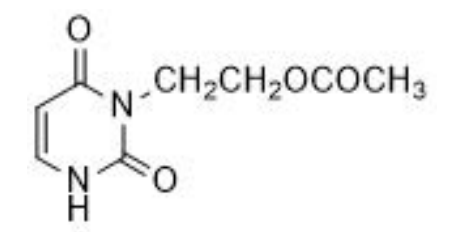

3j

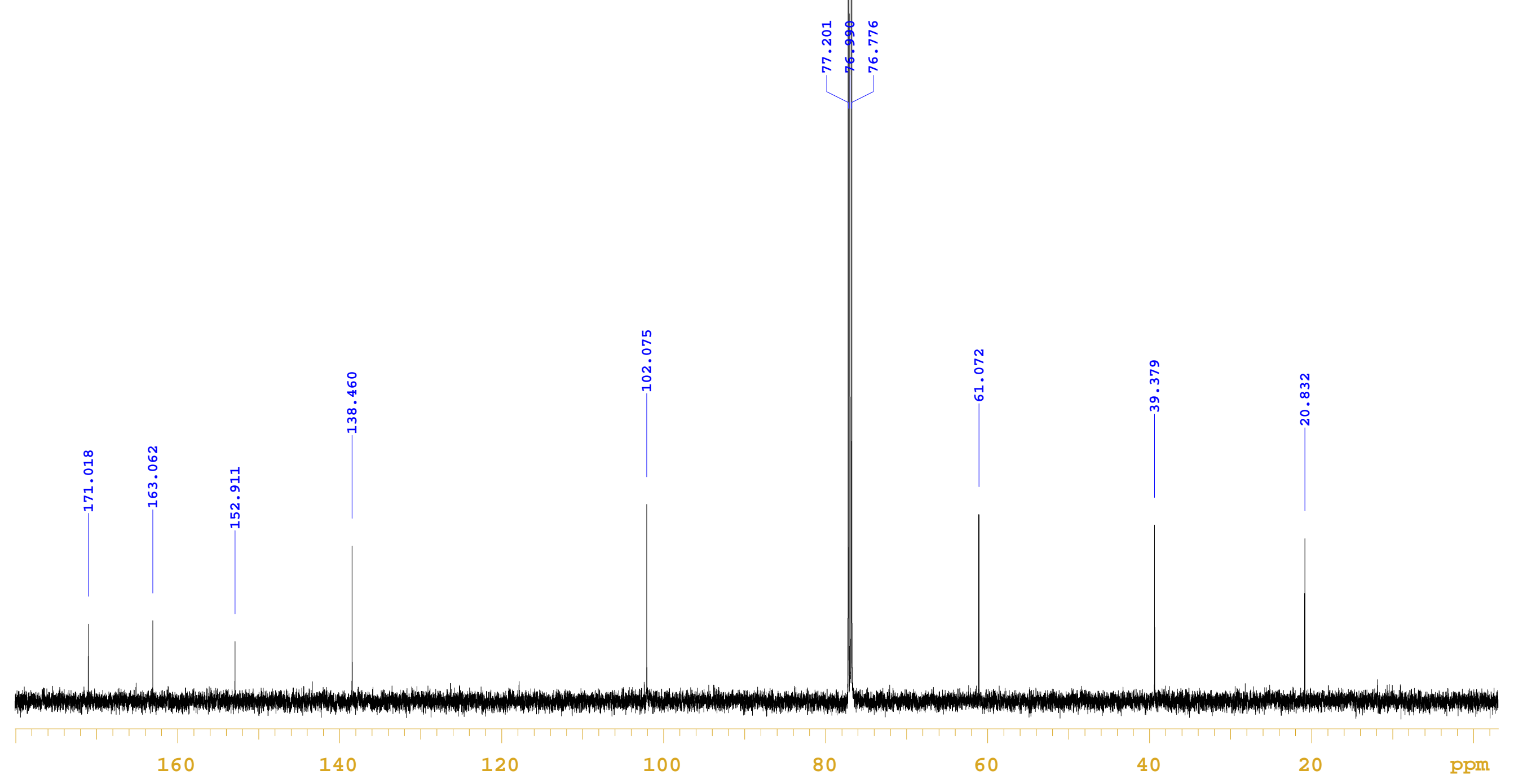


1H NMR, 600MHz, CDCl3
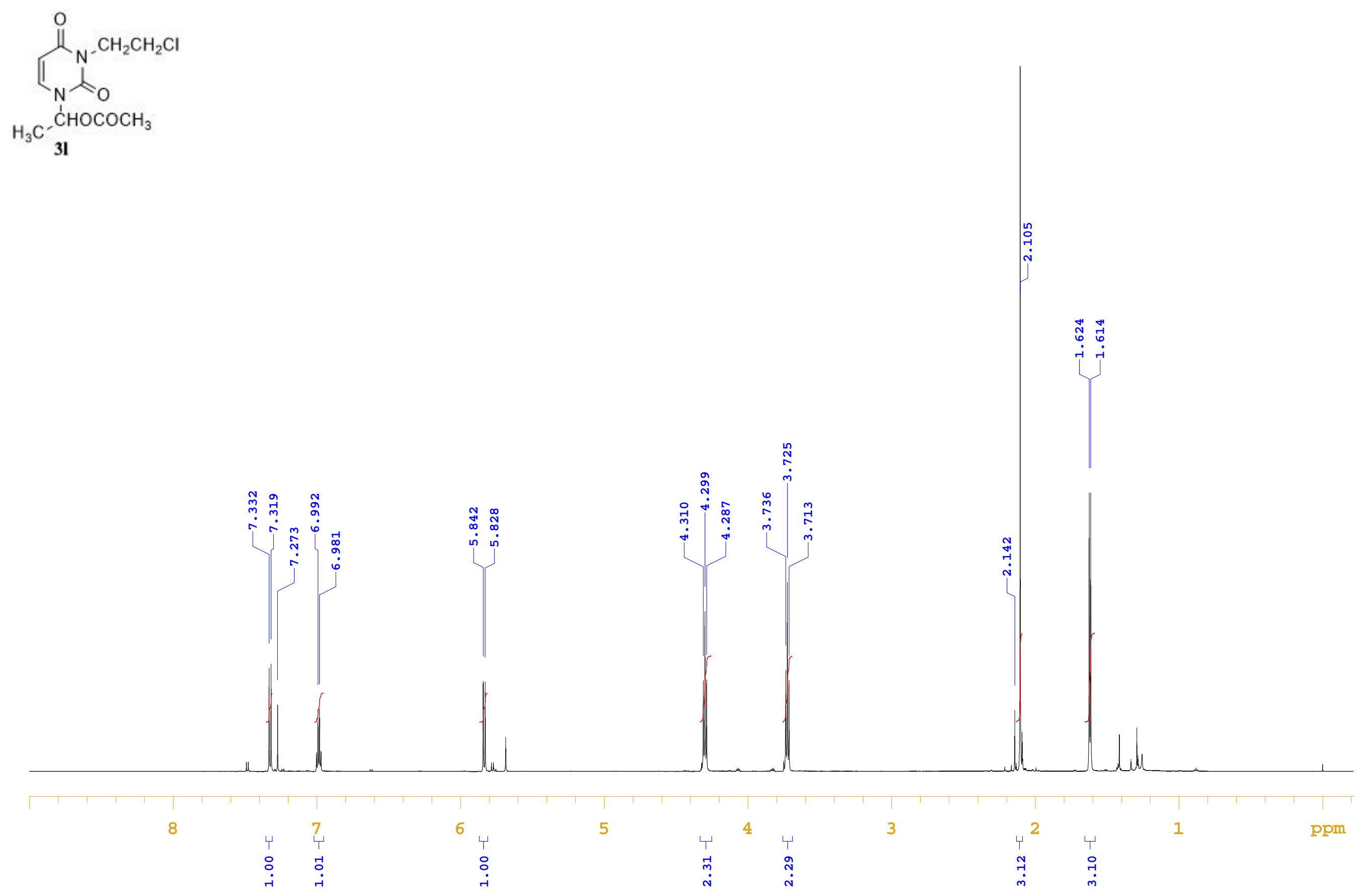
$13 \mathrm{C}\{1 \mathrm{H}\} \mathrm{NMR}, 150 \mathrm{MHz}, \mathrm{CDCl} 3$
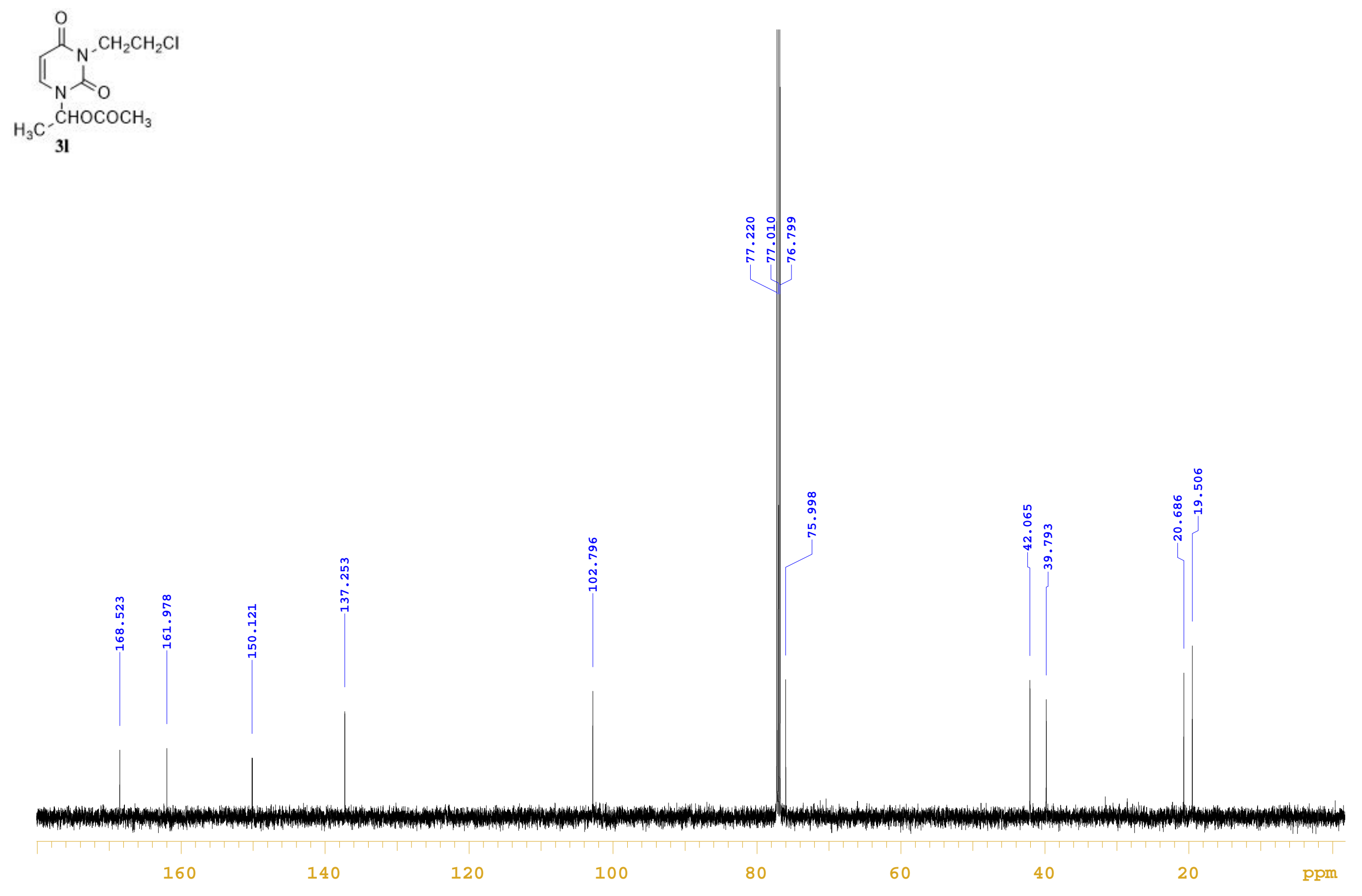
1H NMR, 600MHz, CDCl3

3n

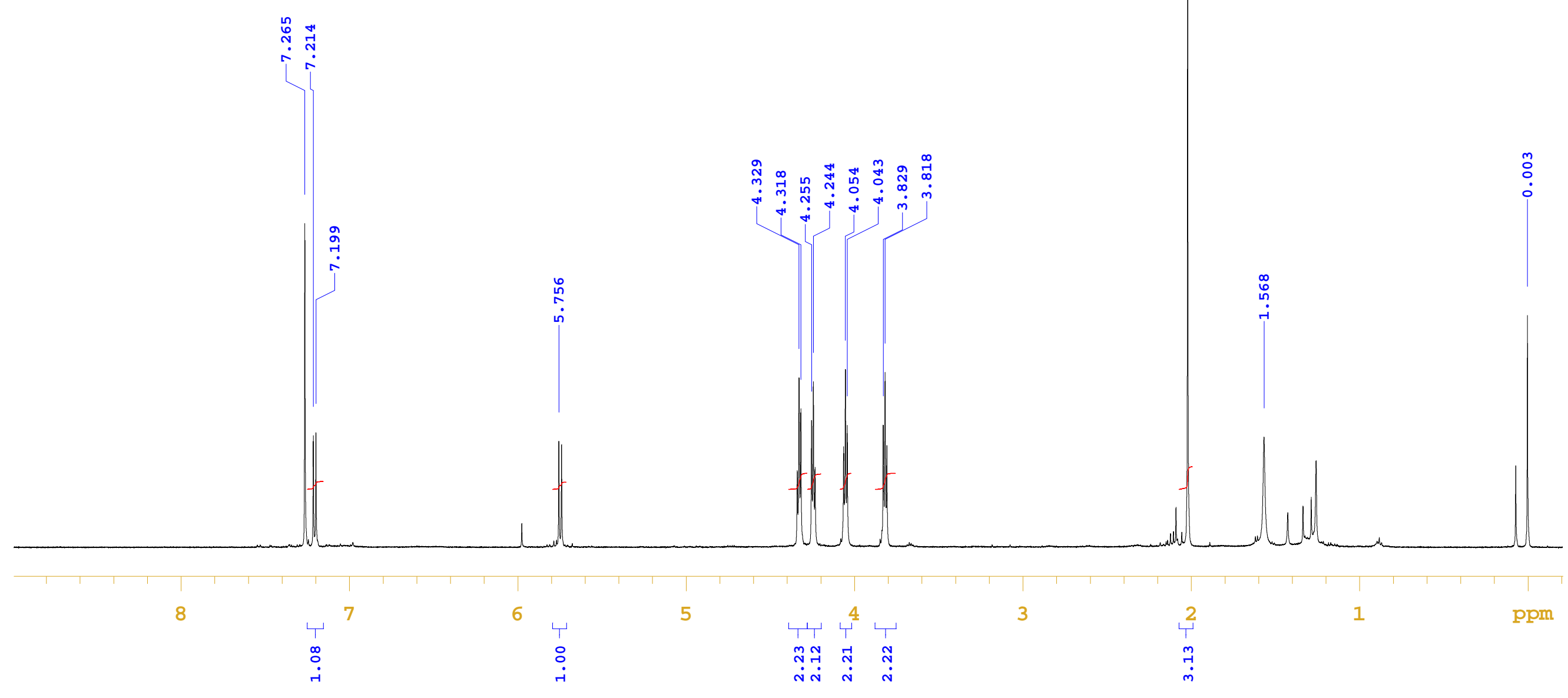


$13 \mathrm{C}\{1 \mathrm{H}\} \mathrm{NMR}, 150 \mathrm{MHz}, \mathrm{CDCl} 3$

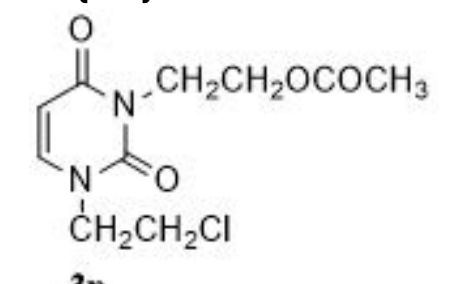

$3 n$

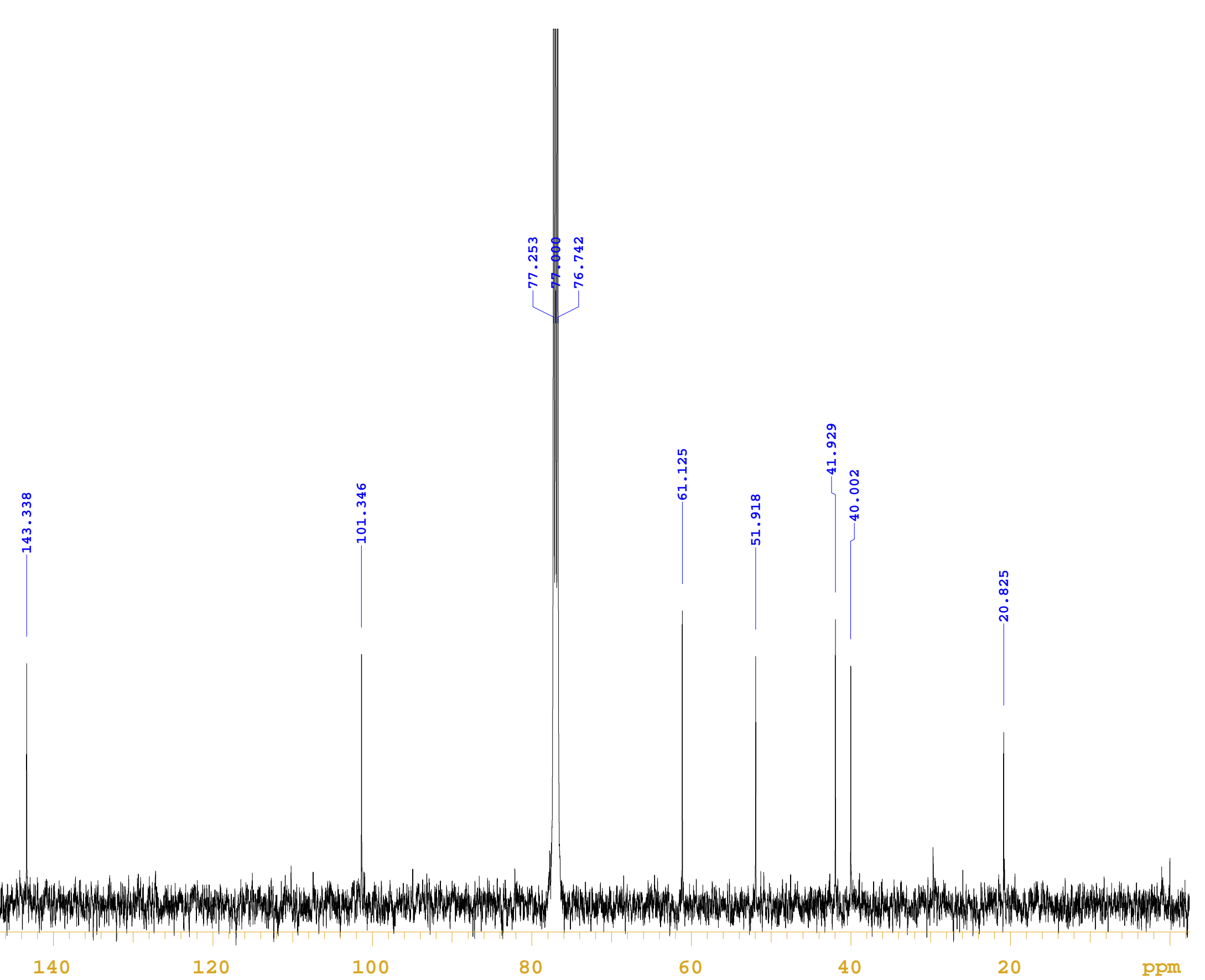


1H NMR, 600MHz, CDCl3
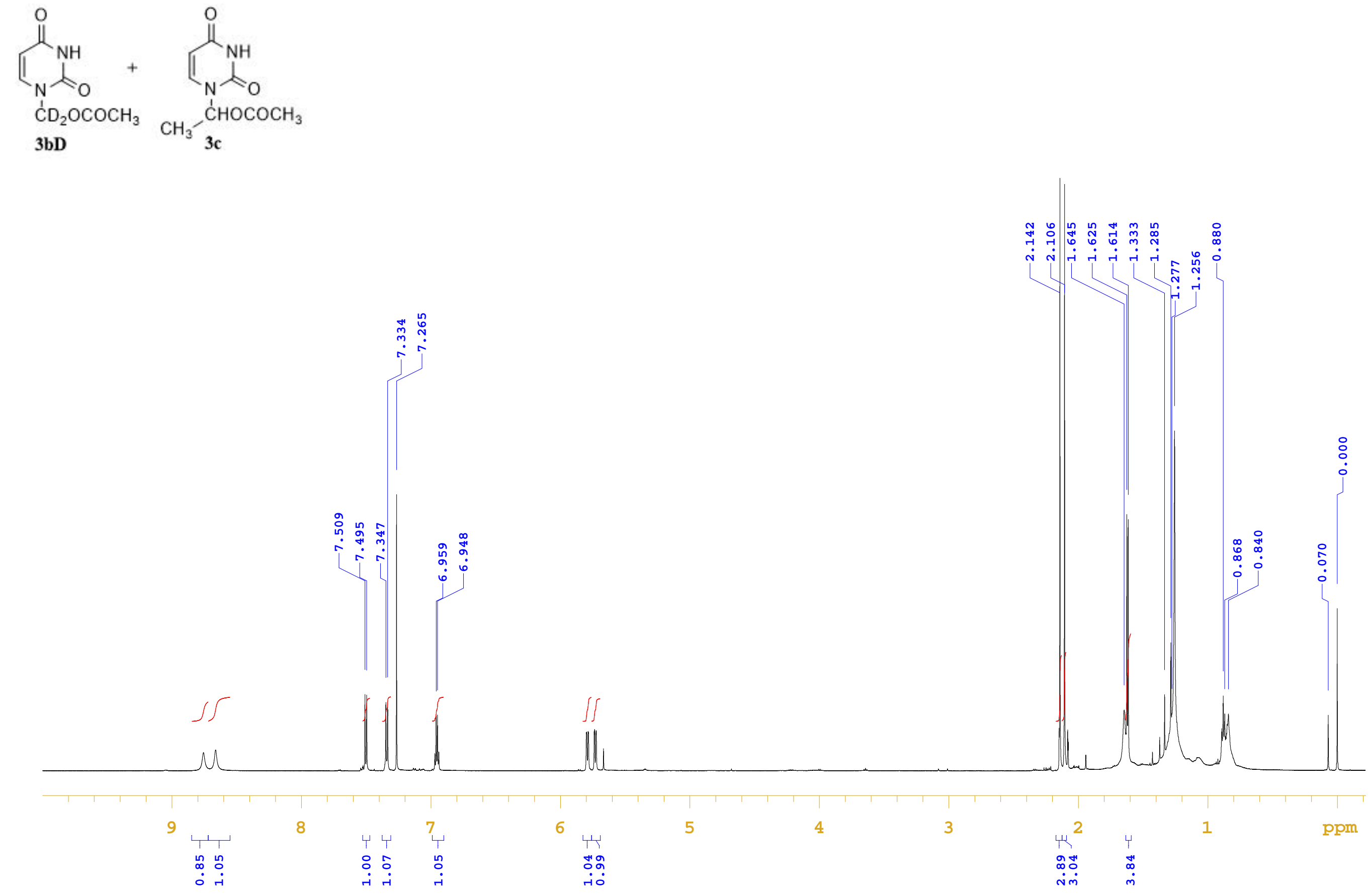
$13 \mathrm{C}\{1 \mathrm{H}\} \mathrm{NMR}, 150 \mathrm{MHz}, \mathrm{CDCl} 3$
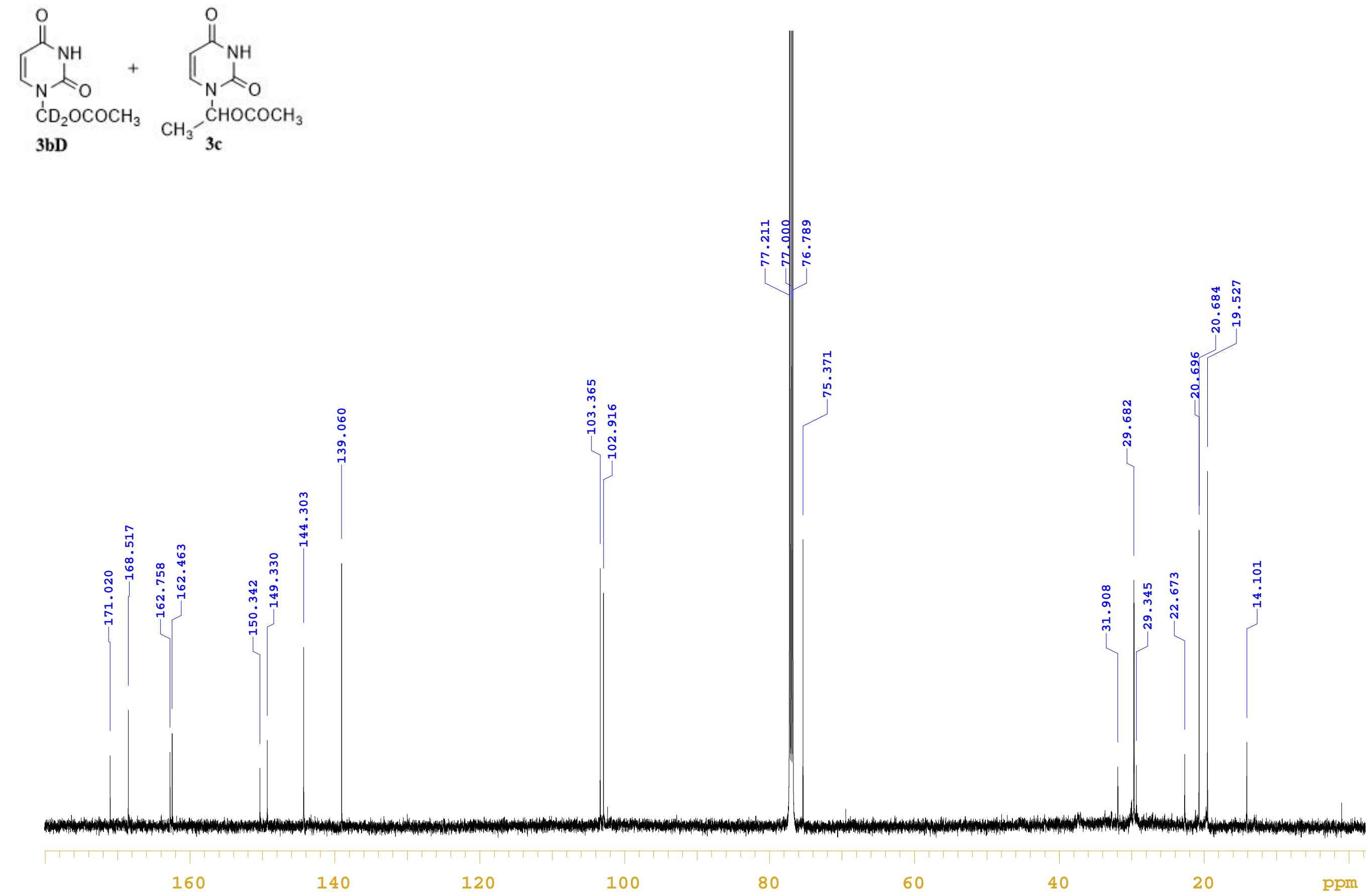


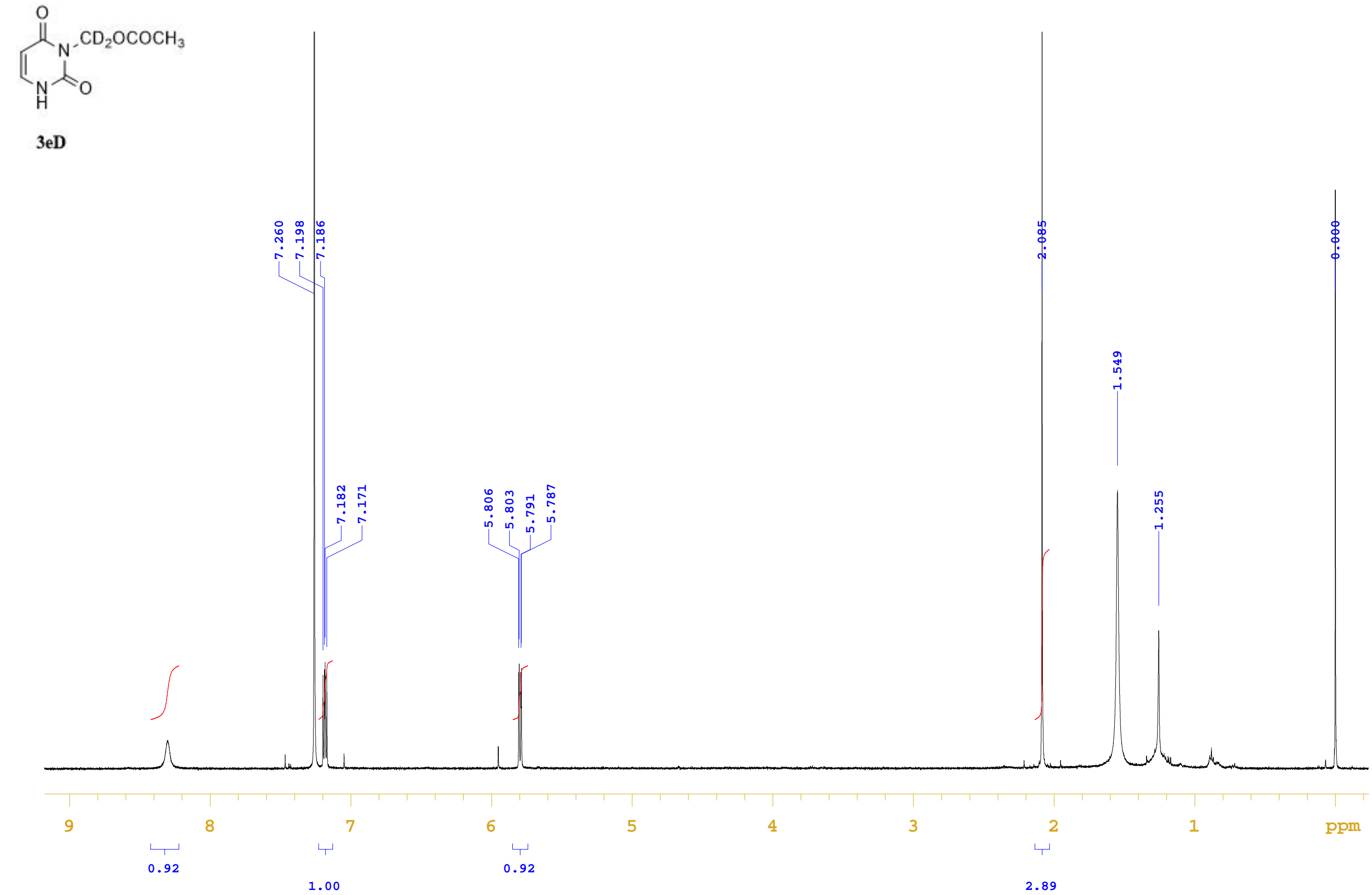


$13 \mathrm{C}\{1 \mathrm{H}\} \mathrm{NMR}, 150 \mathrm{MHz}, \mathrm{CDCl} 3$

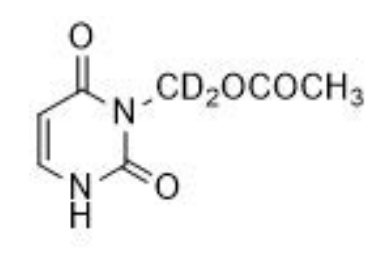

3eD

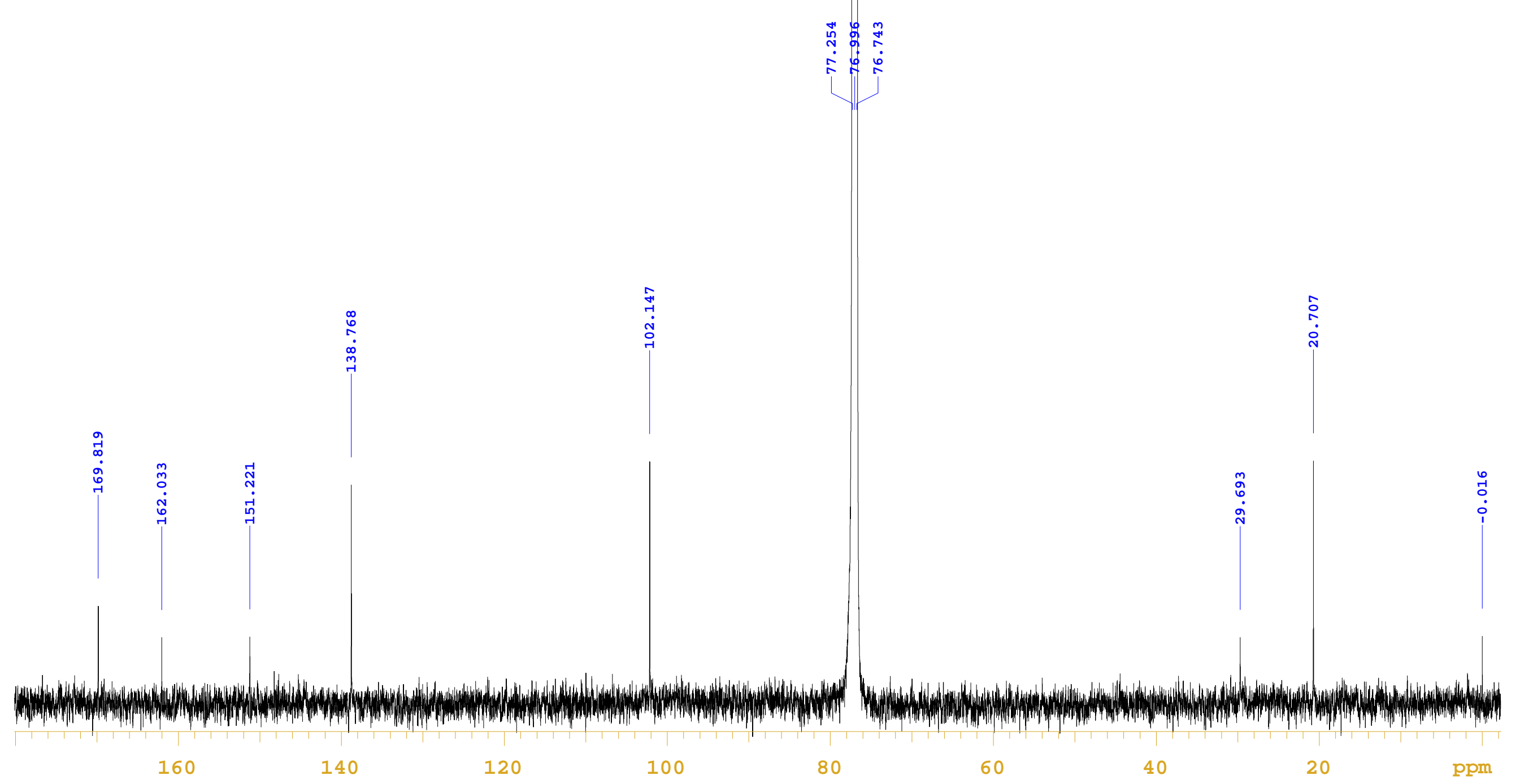


1H NMR, $600 \mathrm{MHz}, \mathrm{CDCl} 3$

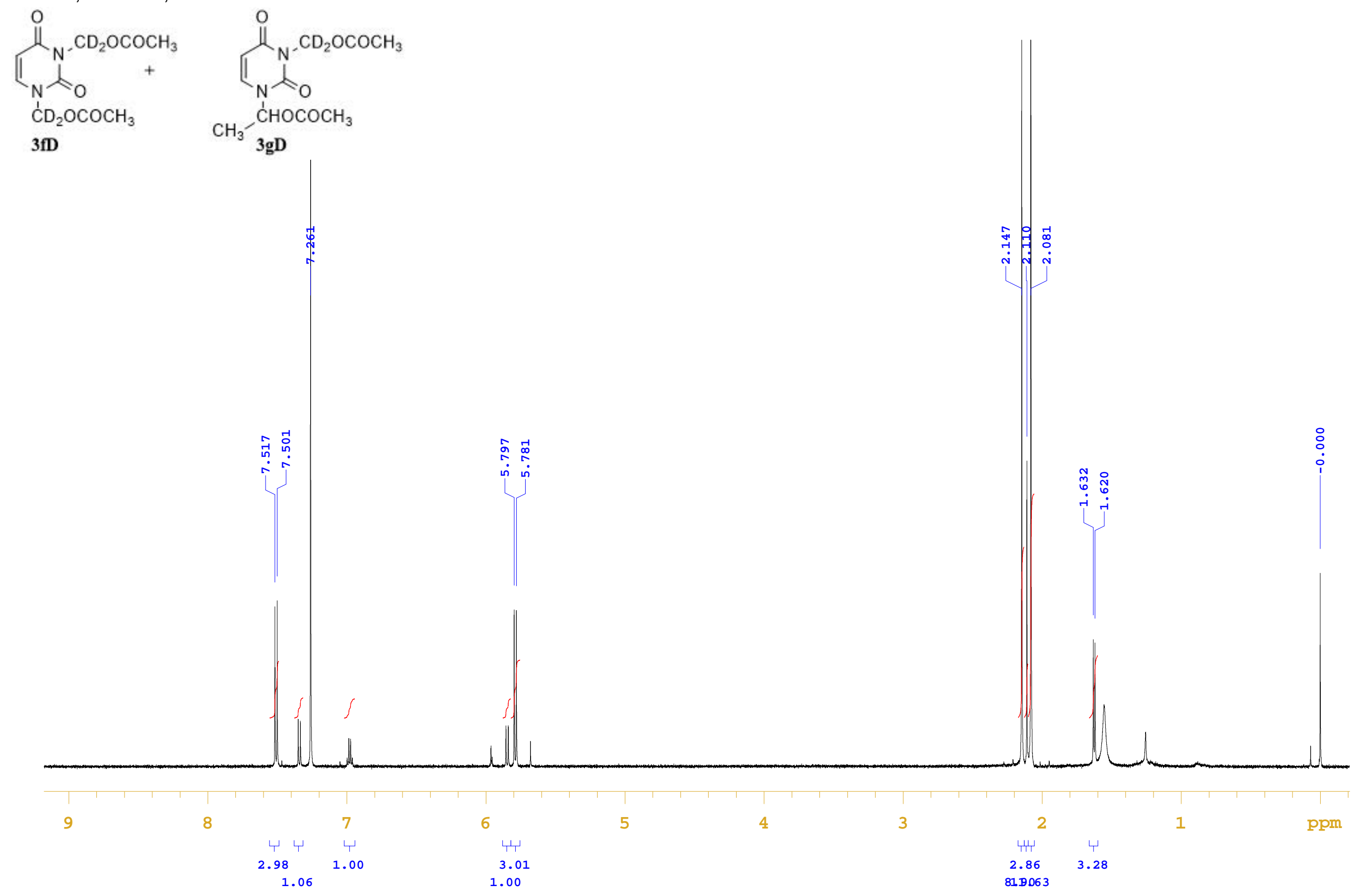


$13 \mathrm{C}\{1 \mathrm{H}\} \mathrm{NMR}, 150 \mathrm{MHz}, \mathrm{CDCl} 3$
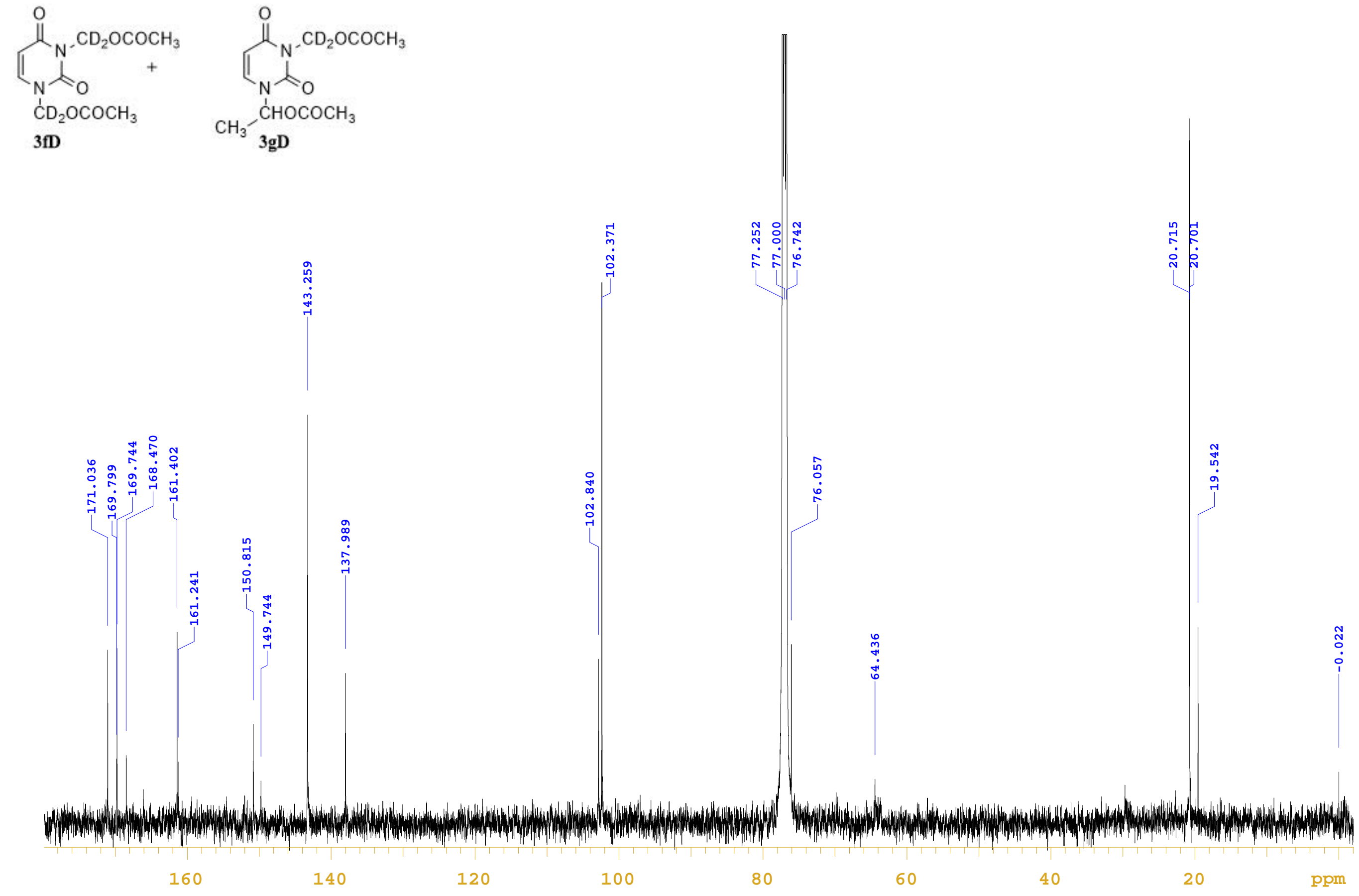


\section{checkCIF/PLATON report}

You have not supplied any structure factors. As a result the full set of tests cannot be run.

THIS REPORT IS FOR GUIDANCE ONLY. IF USED AS PART OF A REVIEW PROCEDURE FOR PUBLICATION, IT SHOULD NOT REPLACE THE EXPERTISE OF AN EXPERIENCED CRYSTALLOGRAPHIC REFEREE.

No syntax errors found. CIF dictionary Interpreting this report

\section{Datablock: 2020_02_24_dt_kt-296-55-3-2_k}

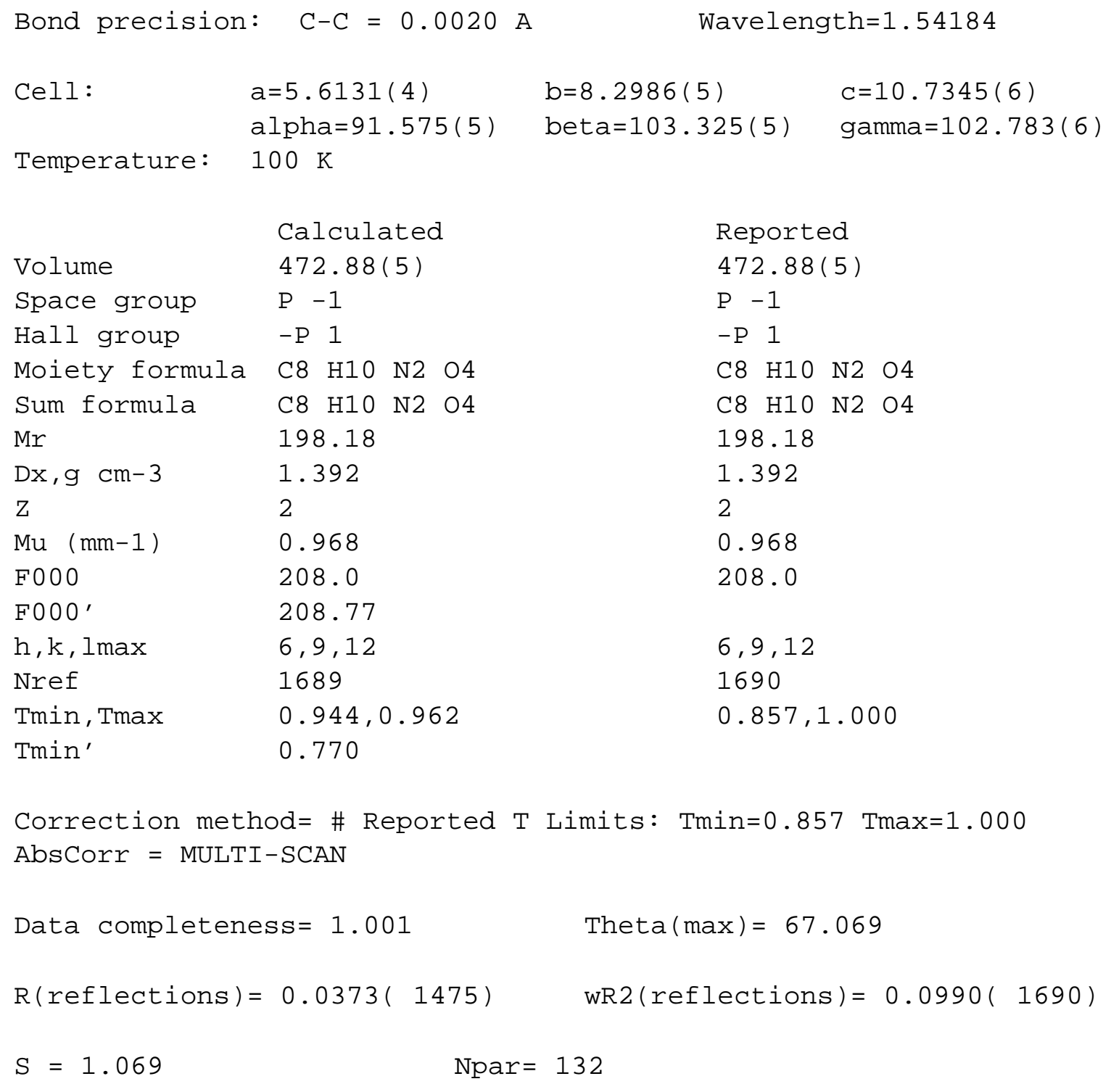


Alert level $\mathbf{G}$

PLAT002_ALERT_2_G Number of Distance or Angle Restraints on AtSite PLAT012_ALERT_1_G No__shelx_res_checksum Found in CIF ......

PLAT172_ALERT_4_G The CIF-Embedded .res File Contains DFIX Records

PLAT793_ALERT_4_G Model has Chirality at C7 (Centro SPGR)

PLAT860_ALERT_3_G Number of Least-Squares Restraints ..........
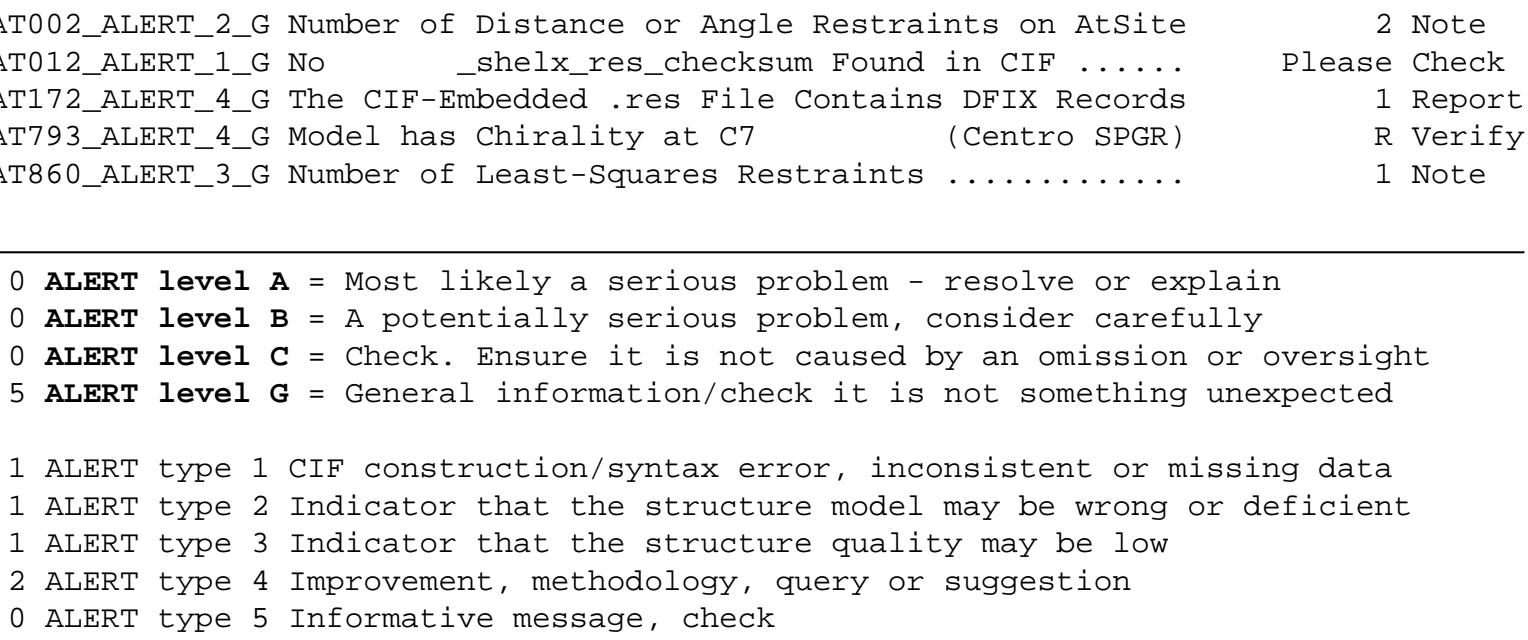

It is advisable to attempt to resolve as many as possible of the alerts in all categories. Often the minor alerts point to easily fixed oversights, errors and omissions in your CIF or refinement strategy, so attention to these fine details can be worthwhile. In order to resolve some of the more serious problems it may be necessary to carry out additional measurements or structure refinements. However, the purpose of your study may justify the reported deviations and the more serious of these should normally be commented upon in the discussion or experimental section of a paper or in the "special_details" fields of the CIF. checkCIF was carefully designed to identify outliers and unusual parameters, but every test has its limitations and alerts that are not important in a particular case may appear. Conversely, the absence of alerts does not guarantee there are no aspects of the results needing attention. It is up to the individual to critically assess their own results and, if necessary, seek expert advice.

\section{Publication of your CIF in IUCr journals}

A basic structural check has been run on your CIF. These basic checks will be run on all CIFs submitted for publication in IUCr journals (Acta Crystallographica, Journal of Applied Crystallography, Journal of Synchrotron Radiation); however, if you intend to submit to Acta Crystallographica Section C or E or IUCrData, you should make sure that full publication checks are run on the final version of your CIF prior to submission.

\section{Publication of your CIF in other journals}

Please refer to the Notes for Authors of the relevant journal for any special instructions relating to CIF submission.

\section{PLATON version of 22/12/2019; check.def file version of 13/12/2019}


Datablock 2020_02_24_dt_kt-296-55-3-2_k - ellipsoid plot

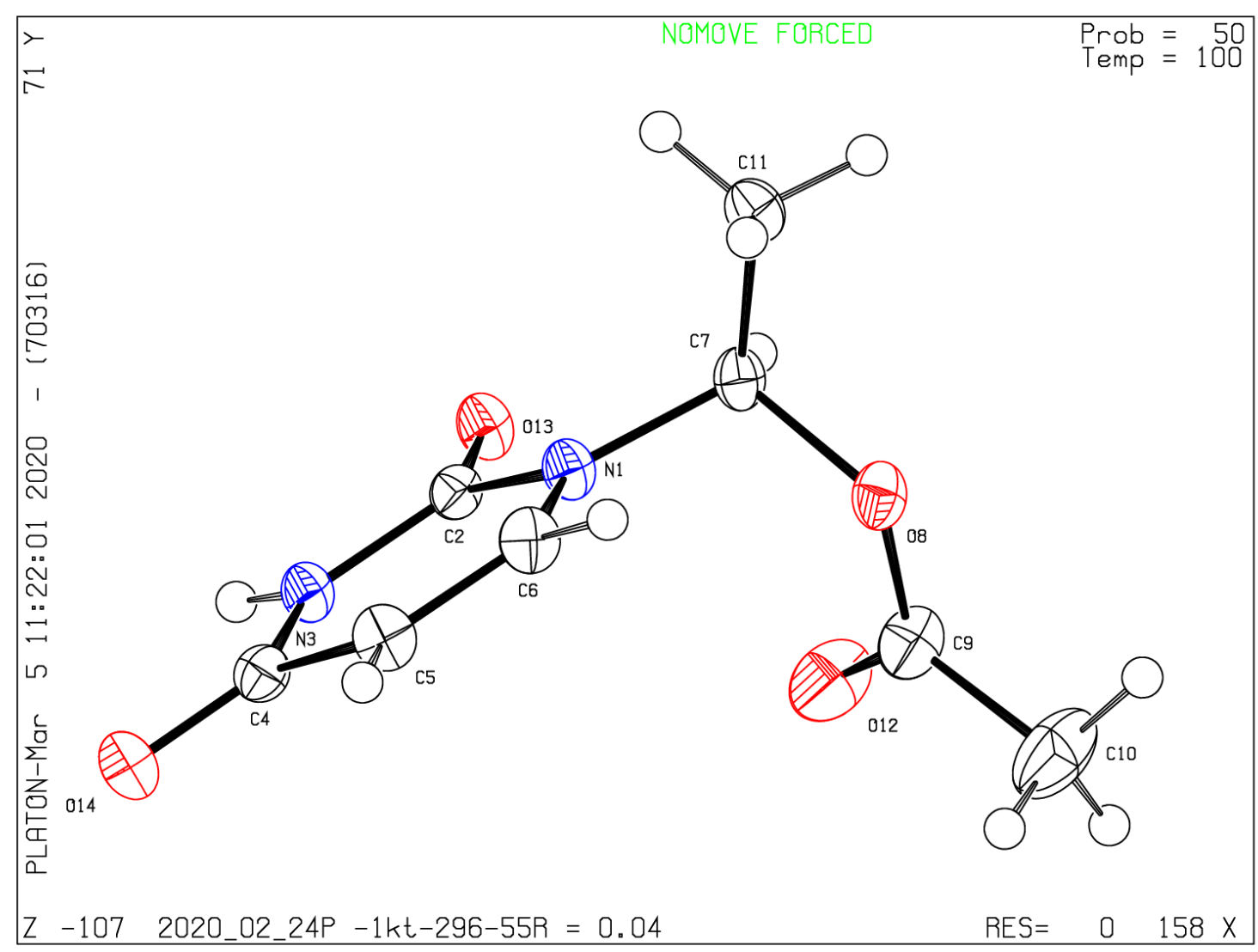

